TRANSACTIONS OF THE

AMERICAN MATHEMATICAL SOCIETY

Volume 348, Number 10, October 1996

\title{
TRANSFINITE MULTIFRACTAL DIMENSION SPECTRUMS
}

\author{
STANLEY C. WILLIAMS
}

\begin{abstract}
The first order theory of the decomposition of measures with respect to dimension which has been developed by Kahane, Katznelson, Cutler, and others is extended through transfinite recursion to a $\omega_{1}$-order theory. Necessary and sufficient conditions for a finite regular Borel measure on $[0, d]]_{1}^{\omega_{1}}$ to be a $\omega_{1}$-order multispectrum for a finite Borel measure on $\mathbb{R}^{d}$ is given.
\end{abstract}

\section{INTRODUCTION}

The purpose of this paper is to introduce a theory of transfinite multispectral decompositions of measures with respect to dimension which is the transfinite version of the first order theory that is present in the work of Cutler, Kahane and Katznelson. The main goal is to identify the set of all possible $\omega_{1}$-order multispectrums. The first order version of this theory has been given by Cutler in Corollary 4.4.1 of [4]. Kahane and Katznelson, in the examples of [10], have also indicated some special specifications of unidimensionality of the kernel obtained in the first order decomposition. In this paper a complete characterization of possible $\omega_{1}$ multispectrums in $\mathbb{R}^{d}$ in terms of 'basic multispectrums' will complete certain ideas started by Kahane and Katznelson in their examples. More generally, necessary and sufficient conditions for a finite regular Borel measure on $[0, d]^{\omega_{1}}$ to be the $\omega_{1}$-order multispectrum of a finite measure in $\mathbb{R}^{d}$ will be given in what shall be called the Multispectral Theorem (Theorem 9.2). The paper is divided into several sections. Section 2 reviews the first order decomposition theory as given by Cutler, Kahane and Katznelson. Other types of first order multifractal formalisms can be found in Michon/Peyrière [13], Olsen [14], and Holley/Waymire [6]. In Section 3, the transfinite multispectral extensions, $\alpha$-order multispectrums, and $\alpha$-order local dimension maps are defined. Section 4 gives the First Version of Necessity of the Multispectral Theorem. Section 5 introduces the ideas of basic multispectrums and gives the Second Version of Necessity of the Multispectral Theorem. Section 6 introduces some linearity results and closure properties of the set of all $\omega_{1}$-order multispectrums and ends with a uniqueness characterization for a measure being the $\alpha$-order multispectral extension of a specified measure. Section 7 begins the investigation of sufficiency by considering random multiplicative cascades in certain ultrametric spaces. That section ends with Theorem 7.7 which states that if certain filtration systems can be found, then randomly generated measures give all the 'bits and pieces' needed in proving sufficiency. This uses the dependent cascade theory of Waymire and Williams in [19][21][18][20] which fits into the positive martingale

Received by the editors January 29, 1995.

1991 Mathematics Subject Classification. Primary 28A78, 28A80, 60G42; Secondary 60G57 .

Key words and phrases. Multifractals, dimension spectra, martingales, random measures.

(C)1996 American Mathematical Society 
theory of Kahane found in [8][9]. These processes are generalization of the Mandelbrot martingales studied by Kahane and Peyrière in [11]. Section 8 shows that all the filtration bases needed in Section 7 can be constructed. Finally Section 9 gives the complete statement of the Multispectrum Theorem for $\mathbb{R}^{d}$ (Theorem 9.2).

\section{First Order Theory}

Let $(T, \rho)$ be a fixed compact metric space, and $M^{+}(T)$ be the set of all finite Borel measures on $T$. For each $\mu \in M^{+}(T), t \in T$, and $a \in(0, \infty]$, the $a$-potential of $\mu$ at $t, U_{\mu}^{a}(t)$, is the integral $\int \rho(t, s)^{-a} d \mu(s)$. The $a$-energy, $I_{\mu}^{a}$, of $\mu$ is defined by $\int U_{\mu}^{a}(t) d \mu(t)$. A Borel subset $E$ of $T$ is said to have positive $a$-capacity if there exists a measure $\mu \in M^{+}(X)$ so that the $I_{\mu}^{a}<\infty$ and $\mu(E)>0$. The set $E$ is said to have null $a$-capacity if no such measure exists. The capacitarian dimension of $E, \psi(E)$, is defined to be the $\inf \{a \in[0, \infty): E$ has null $a$-capacity $\}=$ $\sup \{a \in[0, \infty): E$ has positive $a$-capacity $\}$. The Hausdorff dimension of a set is known to be the same as its capacitarian dimension. See Kahane [7] (p. 133). Two obvious facts about the function $\psi$ are:

(1) If $A \subseteq B$, then $\psi(A) \leq \psi(B)$.

(2) $\psi\left(\bigcup A_{i}\right)=\sup \psi\left(A_{i}\right)$ for any countable collection of Borel sets $\left\{A_{i}\right\}$.

Associated with the dimension function $\psi$ is the local dimension function $\Phi: M^{+}(T) \times T \rightarrow[0, \infty]$ defined by

$$
\Phi(\mu, t)=\inf \left\{\alpha \in[0, \infty): U_{\mu}^{\alpha}(t)=\infty\right\} .
$$

This function is almost equal to the dimension concentration map studied by Cutler [4].

Our first task is to review and expand on the theory concerning the local dimension function $\Phi$ and the kernel decomposition associated with it. This theory is called the first order theory of decompositions with respect to dimension. In particular, the first two parts of the next theorem (Theorem 2.1) can be found in the papers by Kahane and Katznelson (Lemmas 2 and 3) [10] and by Cutler [4]. Part 3 follows easily from the first two parts and implies a certain linearity. (See Theorem 6.1). Further, part 3 implies, in the case $\psi(T)<\infty$, the fact that the dimension-moment functional $\psi^{*}: M^{+}(T) \rightarrow[0, \infty)$ defined by

$$
\psi^{*}(\mu)=\int \Phi(\mu, t) d \mu(t)
$$

is linear, in the sense of additive and positive homogeneous, and that $\psi$ can be essentially recovered from $\psi^{*}$. See Conway [3] (Theorem 5.12). Some relationships between strong linear functionals on $M^{+}(T)$ and set functions has been studied by Mauldin [12]. See also Remark 9.3.

The collection of all continuous real-valued functions on $T$ will be denoted by $C(T)$. The weak* topology on $M^{+}(T)$ is the weakest topology which makes the functionals ' $\mu \rightarrow \int f d \mu$ ' continuous for all $f \in C(T)$. The Borel field of $M^{+}(T)$ with respect to the weak* topology will be denoted by $\mathcal{B}\left(M^{+}(T)\right)$. The product topology on $M^{+}(T) \times T$ where the topology of the first factor is the weak ${ }^{*}$ topology and the second is the metric $\rho$-topology will be called the weak topology. Let $\mathbb{N}$ denote the natural counting numbers.

Theorem 2.1. The function $\Phi$ is weakly Borel and for $x \in[0, \infty]$, and for $\mu$, $\nu \in M^{+}(T):$ 
(1) the dimension of $\Phi(\mu, \cdot)^{-1}[0, x]$ is $\leq x$,

(2) if $K$ is a Borel subset of $\Phi(\mu, \cdot)^{-1}(x, \infty]$ and $\mu(K)>0$, then the dimension of $K$ is $>x$,

(3) if $\nu \ll \mu$, then $\nu$-a.e., $\Phi(\nu, \cdot)=\Phi(\mu, \cdot)$.

Proof. For $x>0, \Phi^{-1}(-\infty, x)=\left\{\langle\mu, t\rangle: \exists y<x, y\right.$ rational, s.t. $\left.U_{\mu}^{y}(t)=\infty\right\}=$ $\left\{\langle\mu, t\rangle: \exists y<x, y\right.$ rational s.t. $\forall M \in \mathbb{N}, \exists N \in \mathbb{N}$, s.t. $\left.\int\left(\rho^{-y}(s, t) \wedge N\right) d \mu(s) \geq M\right\}$. Since the map ' $\langle s, t\rangle \rightarrow \rho^{-y}(s, t) \wedge N$ ' is uniformly continuous on $T \times T,\langle\mu, t\rangle \rightarrow$ $\int \rho^{-y}(s, t) \wedge N d \mu(s)$ ' is weakly continuous. Thus $\Phi$ is weakly Borel.

Part (1) is essentially Lemma 3 of [10]. Let $F_{y}=\left\{t: U_{\mu}^{y}(t)=\infty\right\}$. Lemma 3 of [10] states $F_{y}$ has null $y$-capacity and so the dimension of $F_{y}$ is less than or equal to $y$. But $\Phi(\mu, \cdot)^{-1}[0, x] \subseteq F_{y}$ if $y>x$. By property 1 of $\psi$, the dimension of $\Phi(\mu, \cdot)^{-1}[0, x]$ is $\leq y$ for all $y>x$.

For (2), note that for every $t \in \Phi(\mu, \cdot)^{-1}(x, \infty]$, there exists a $a_{t}>x$ so that $U_{\mu}^{a_{t}}(t)<\infty$. Let $A_{M}$ denote the Borel set $\left\{t \in K: U_{\mu}^{x+M^{-1}}(t)<M\right\}$. Easily, there exists an $M<\infty$, so that $\mu\left(A_{M}\right)>0$. Thus, $I_{\mu 1}^{x+M_{A_{M}}}<\infty$, implying by the definition of $\psi$ and property (1) that $x+M^{-1}<\psi\left(A_{M}\right) \leq \psi(K)$.

For (3), note for any real $x$, part (1) implies $\Phi(\mu, \cdot)^{-1}[0, x]$ has dimension $\leq x$, implying by part (2) that

$$
\nu\left(\left(\Phi(\mu, \cdot)^{-1}[0, x]\right) \cap\left(\Phi(\nu, \cdot)^{-1}(x, \infty]\right)\right)=0 .
$$

Likewise, $\mu\left(\left(\Phi(\nu, \cdot)^{-1}[0, x]\right) \cap\left(\Phi(\mu, \cdot)^{-1}(x, \infty]\right)\right)=0$. Since $\nu \ll \mu$, this last implies

$$
\nu\left(\left(\Phi(\nu, \cdot)^{-1}[0, x]\right) \cap\left(\Phi(\mu, \cdot)^{-1}(x, \infty]\right)\right)=0 .
$$

Combining (2.2) and (2.3), we obtain for all $x$,

$$
\nu\left(\left(\Phi(\nu, \cdot)^{-1}[0, x]\right) \cap\left(\Phi(\mu, \cdot)^{-1}[0, x]\right)\right)=0,
$$

from which follows (3).

It is not hard to show that for any $\mu$ and $\nu$ in $M^{+}(T)$,

$$
\Phi(\mu+\nu, \cdot)=\Phi(\mu, \cdot) \wedge \Phi(\nu, \cdot) .
$$

The function $\Phi$ allows us to define a first order dimension spectrum and a first order spectral extension distribution for any $\mu$ in $M^{+}(T)$.

Definition 2.1. The function $\sigma: M^{+}(T) \rightarrow M^{+}([0, \infty])$ defined by $\sigma(\mu)=\mu \circ$ $\Phi(\mu, \cdot)^{-1}$ is called the first order dimension spectrum map. The measure $\sigma(\mu)$ is called the first order dimension spectrum of $\mu$. Define the first order extension map $K: M^{+}(T) \rightarrow M^{+}(T \times[0, \infty])$ by

$$
K(\mu)=\mu \circ(\langle i d, \Phi(\mu, \cdot)\rangle)^{-1}
$$

where $i d: T \rightarrow T$ is the identity on $T$ and where $\langle i d, \Phi(\mu, \cdot)\rangle$ is the function from $T$ into $T \times[0, \infty]$ defined by $\langle i d, \Phi(\mu, \cdot)\rangle(t)=\langle t, \Phi(\mu, t)\rangle$. The measure $K(\mu)$ is called the first order spectral extension distribution of $\mu$.

The measure $\sigma(\mu)$ is called the Kahane/Katznelson spectral measure in [21] and the dimension measure of $\mu$ by Cutler. It will be shown in Theorem 6.1 that Theorem 2.1 part (3) implies both $\sigma$ and $K$ are strongly continuous and linear.

The following two definitions are made for notational convenience: 
Definition 2.2. If $\phi:(T, \rho) \rightarrow(X, \Sigma)$ is a measurable map and $\mu \in M^{+}(T)$, then a decomposition kernel of $\mu$ with respect to $\phi$ is a $\mu \circ \phi^{-1}$-essentially defined map ' $x \rightarrow \mu_{\phi=x}$ ' from $X$ into $\operatorname{Pr}(T)$ which is $(X, \Sigma)$ to $\left(M^{+}(T), \mathcal{B}\left(M^{+}(T)\right)\right)$ measurable and satisfies: for any bounded $\mathcal{B}(T) \times(X, \Sigma)$ measurable map $G$ : $T \times X \rightarrow \mathbb{R}$,

$$
\int G(t, \phi(t)) d \mu(t)=\iint G(t, x) d \mu_{\phi=x}(t) d \mu(x) .
$$

Other notations used for this kernel are: $\left\langle\mu_{\phi=x}\right\rangle_{x \in X}$ and $\langle\mu(\cdot \mid \phi=x)\rangle_{x \in X}$.

If $\mu$ is a probability measure and $X$ is a compact metric space with $\Sigma=\mathcal{B}(X)$, then ' $x \rightarrow \mu_{\phi=x}$ ' is a regular conditional probability. See Proposition 46.3 in Parthasarathy [15]. A special case of the above disintegration in product spaces of compact metric spaces gives rise to the following:

Definition 2.3. If both $(T, \rho)$ and $(X, \sigma)$ are compact metric spaces, $\pi_{T}$ and $\pi_{X}$ are the coordinate projections of $T \times X$ onto $T$ and $X$ respectively, and $\mu \in$ $M^{+}(T \times X)$, then the factor decomposition kernel of $\mu$ with respect to $\pi_{X}$ is the $\mu \circ \pi_{X}^{-1}$-essentially unique map ' $x \rightarrow \mu_{x}$ ' from $X$ into $\operatorname{Pr}(T)$ defined by $\mu_{x}=\mu_{\pi_{X}=x} \circ \pi_{T}^{-1}$, where ' $x \rightarrow \mu_{\pi_{X}=x}$ ' is the decomposition kernel of $\mu$ with respect to $\pi_{X}$ as in Definition 2.2.

Finally, it is convenient in places to represent measures through integrals of measures. The following notation will be used:

Definition 2.4. Let $(T, \rho)$ be a compact metric space, $(X, \Sigma)$ is a measurable space, and ' $x \rightarrow \mu_{x}$ ' is a $(X, \Sigma)$ to $\left(M^{+}(T), \mathcal{B}\left(M^{+}(T)\right)\right.$ measurable function and $\kappa$ is a finite measure on $\Sigma$ so that ' $x \rightarrow \mu_{x}(T)$ ' is in $L^{1}(\kappa)$, then the unique measure $\mu \in M^{+}(T)$ so that for every $H \in C(T)$,

$$
\int H d \mu=\iint H(t) d \mu_{x}(t) d \kappa(x)
$$

will be called the weak* integral, $w^{*}-\int \mu_{x} d \kappa(x)$.

Easily, $\mu=w^{*}-\int \mu_{\phi=x} d \mu \circ \phi^{-1}(x)$ in the setting of Definition 2.2 .

For each $\mu \in M^{+}(T)$, let ' $x \rightarrow K(\mu)_{x}$ ' denote the factor kernel of $K(\mu)$ with respect to the projection $\zeta: T \times[0, \infty] \rightarrow[0, \infty]$. Clearly, for $\sigma(\mu)$-a.e.- $x, K(\mu)_{x}=$ $\mu_{\Phi(\mu, \cdot)=x}$ where ' $x \rightarrow \mu_{\Phi(\mu, \cdot)=x}$ ' is the decomposition kernel of $\mu$ with respect to $\Phi(\mu, \cdot)$.

The following theorem is one of the spectral decomposition theorems of Kahane/Katznelson and of Cutler, and forms the second main theorem of this section.

Theorem 2.2. Let $\mu \in M^{+}(T), \sigma=\sigma(\mu)$ and let ' $s \rightarrow K(\mu)_{s}$ ' denote the factor kernel of $K(\mu)$ with respect to the projection $\zeta: T \times[0, \infty] \rightarrow[0, \infty], s \in[0, \infty]$. Then the following are true:

(a) $\mu=w^{*}-\int K(\mu)_{s} d \sigma(s)$ where $w^{*}-\int K(\mu)_{s} d \sigma(s)$ is a weak integral,

(b) for all $x \in[0, \infty]$, the measure $w^{*}-\int_{[0, x]} K(\mu)_{s} d \sigma(s)$ is supported on a set $B$ with $\psi(B) \leq x$,

(c) the measure $w^{*}-\int_{(x, \infty]} K(\mu)_{s} d \sigma(s)$ gives the set $A$ measure zero if $\psi(A) \leq x$.

Further, if $\zeta$ is a finite Borel measure on $[0, \infty]$ and ' $x \rightarrow \nu_{x}$ ' is a weak $k^{*}$ Borel map from $[0, \infty]$ into the probability measures on $T$ so that $(a),(b)$, and (c) are satisfied with $\sigma$ being replaced by $\zeta$ and the kernel ' $x \rightarrow K(\mu)_{x}$ ' being replaced by ' $x \rightarrow \nu_{x}$ ', then $\zeta=\sigma$ and $\zeta$-a.e. $-x, \nu_{x}=K(\mu)_{x}$. 
Definition 2.5. A measure $\mu$ in $M^{+}(T)$ is said to be unidimensional of dimension $x$ if $\sigma(\mu)=\delta_{x}$.

More can be said concerning Theorem 2.2: for instance, Cutler has shown that the image of $\sigma$ is $M^{+}([0, d])$ in the case of $T=[0,1]^{d}$. Kahane and Katznelson have shown in the examples of [10] that there exists $\mu$ so that the $K(\mu)_{s}$ are unidimensional of dimension $g(s)$ where $g$ is certain classes of functions. However, they do not specify all the possible examples of what the kernel $\left\langle K(\mu)_{s}\right\rangle_{s}$ can be even in the cases where all the $K(\mu)_{s}$ are restricted to be unidimensional. (what is called in this paper simple order one unidimensionality of order one. See Section 5). We shall attempt to answer this question by repeating the decomposition done above transfinitely and determining necessary and sufficient conditions for a measure to be what will be called a transfinite multispectrum. To do this we need the following first order lemma which is an induction lemma for the transfinite case. It should be noted that all the parts of this lemma have been proved by Cutler in sections of $[4]$.

Theorem 2.3. For any $\mu \in M^{+}(T)$, the following three statements are true:

(1) For $\sigma(\mu)$-a.s.-s, $K(\mu)_{s}$ is supported on a set $B$ with $\psi(B) \leq s$.

(2) If $x$ is an atom of $\sigma(\mu)$, then $K(\mu)_{x}$ is unidimensional of dimension $x$.

(3) If $\mu$ is supported on a set $C$ of dimension $\leq x$, then for $\sigma(\mu)$-a.e.-s, $s \leq x$.

Proof. Let $D$ denote a countable dense subset of $[0, \infty]$. By Theorem 2.2 part (b),for each $x \in D$ there exists a Borel set $B_{x}$ of dimension $x$ so that $w^{*}-\int_{[0, x]} K(\mu)_{s} \sigma(\mu)(s)$ is supported on $B_{x}$. For every $x \in D$, for $\nu$-a.e.- $s \leq x, K(\mu)_{s}$ is supported on $B_{x}$. That is, for $\nu$-a.e.-s, $K(\mu)_{s}$ is supported on $\bigcap\left\{B_{x}: x \in D\right.$ and $\left.s \leq x\right\}$ which by property 1 of $\psi$ implies part (1).

For part (2), note if $x$ is an atom of $\sigma(\mu)$, then $K(\mu)_{x}$ is absolutely continuous with respect to $\mu$. Thus by Theorem 2.1 part (3), $\Phi\left(K(\mu)_{x}, \cdot\right)=\Phi(\mu, \cdot), K(\mu)_{x}$-a.s. Since $K(\mu)_{x}$ is supported on $\Phi(\mu, \cdot)^{-1}\{x\}$, it follows that $K(\mu)_{x}$-a.s., $\Phi(\mu, \cdot)=x$ from which follows, $\Phi\left(K(\mu)_{x}, \cdot\right)=x, K(\mu)_{x}$-a.s. Thus $K(\mu)_{x}$ is unidimensional of dimension $x$.

For (3), note: since $\mu$ is supported on $C$,

$$
\sigma(\mu)(x, \infty]=\left(w^{*}-\int_{(x, \infty]} K(\mu)_{s} d \sigma(\mu)(s)\right)(C)
$$

which by Theorem 2.2 part (c) equals 0 .

This sections ends with some facts which will be referenced as needed in later sections:

If $\nu$ and $\mu$ are finite measures in $M^{+}(T)$ with $\nu \ll \mu$ and $\phi$ is as in Definition 2.2, then $\nu \circ \phi^{-1} \ll \mu \circ \phi^{-1}$, and for $\nu \circ \phi^{-1}$-a.e.- $x, \nu_{\phi=x} \ll \mu_{\phi=x}$. Further, if $T=Y \times Z$, then $\nu \circ \pi_{Z}^{-1} \ll \mu \circ \pi_{Z}^{-1}$ and for the factor kernels of $\nu$ and $\mu$ with respect to the projection $\pi_{Z}$, we have $\nu \circ \pi_{Z}^{-1}$-a.e.- $z, \nu_{z} \ll \mu_{z}$. (In fact, if ' $(y, z) \rightarrow G(y, z)$ ' is the Radon-Nikodym derivative of $\nu$ with respect to $\mu$, then ' $z \rightarrow \int G(y, z) d \mu_{z}(y)$ ' is the Radon-Nikodym derivative of $\nu \circ \pi_{Z}^{-1}$ with respect to $\mu \circ \pi_{Z}^{-1}$, and for $\nu \circ \pi_{Z}^{-1}$-a.e.- $z$,

$$
y \rightarrow G(y, z) \cdot\left\{\int G(y, z) d \mu_{z}(y)\right\}^{-1}
$$

is the Radon-Nikodym derivative of $\nu_{z}$ with respect to $\mu_{z}$.) 
Let $T$ and $X$ be compact metric spaces, $\phi: T \rightarrow X$ and $\psi: T \rightarrow X$ be Borel measurable functions, and $\mu \in M^{+}(T)$. If for every $h \in C(T \times X), \int h(t, \phi(t)) d \mu(t)=$ $\int h(t, \psi(t)) d \mu(t)$, then $\mu$-a.e.,

$$
\phi=\psi
$$

Also in this setting, if $i d: T \rightarrow T$ is the identity map, $\phi: T \rightarrow X$ is measurable as above, $\langle i d, \phi\rangle: T \rightarrow T \times X$ is defined by $\langle i d, \phi\rangle(t)=\langle t, \phi(t)\rangle$, and $\mu \in M^{+}(T)$, then for $\mu \circ \phi^{-1}$-a.e.- $x$,

$$
\mu_{\phi=x}=\left(\mu \circ\langle i d, \phi\rangle^{-1}\right)_{x}
$$

where ' $x \rightarrow \mu_{\phi=x}$ ' is the decomposition kernel of $\mu$ with respect to $\phi$ and ' $x \rightarrow$ $\left(\mu \circ\langle i d, \phi\rangle^{-1}\right)_{x}$, is the factor kernel of $\mu \circ\langle i d, \phi\rangle^{-1}$ with respect to the projection

\section{Definition of Transfinite Multispectrums}

In order to abstract all the information out of the induction lemma, a transfinite sequence of spectral extensions will be defined for each $\mu \in M^{+}(T)$.

Let $\omega_{1}$ be the first uncountable ordinal. Using the usual von Neumann ordinal definition, (see pages 82 and 84 of [1]), if $\alpha, \beta$ are ordinals, then $\alpha<\beta$ if and only if $\alpha \in \beta$. Further there is a $0^{\text {th }}$ ordinal, $\emptyset$. For any ordinal $\alpha, \alpha=\{\gamma: \gamma<\alpha\}$ and $[0, \infty]^{\alpha}$ is the product space which has the ordinals less than $\alpha$ as indices and factors $[0, \infty]$. Elements of $[0, \infty]^{\alpha}$ are denoted by bold letters $\mathbf{s}, \mathbf{u}$, etc. and also by the bracket notation $\left\langle s_{\gamma}\right\rangle_{\gamma<\alpha}$ and $\left\langle u_{\gamma}\right\rangle_{\gamma<\alpha}$. Often if a boldfaced letter such as $\mathbf{s}$ is mentioned as an element of $[0, \infty]^{\alpha}$, and $\gamma<\alpha$, and a non-bold $\gamma$-indexed letter, $s_{\gamma}$, is mentioned in the same context, then the relationship $\mathbf{s}=\left\langle s_{\gamma}\right\rangle_{\gamma<\alpha}$ is assumed to hold and the non-bold letter is taken to be the coordinate of index $\gamma$ of the boldface letter. (Also remember, the lowest index is 0 not 1.)

Definition 3.1 (A Projective System). For each $1 \leq \beta \leq \alpha \leq \omega_{1}$, define projection functions $\pi_{\alpha, \beta}: T \times[0, \infty]^{\alpha} \rightarrow T \times[0, \infty]^{\beta}$ by

$$
\pi_{a, \beta}\left\langle t,\left\langle s_{\gamma}\right\rangle_{\gamma<\alpha}\right\rangle=\left\langle t,\left\langle s_{\gamma}\right\rangle_{\gamma<\beta}\right\rangle \text {. }
$$

For the purposes of this paper, we let $T \times[0, \infty]^{\emptyset}=T$, and $\pi_{\alpha, 0}: T \times[0, \infty]^{\alpha} \rightarrow T$ be the projection defined by $\pi_{a, 0}\left\langle t,\left\langle s_{\gamma}\right\rangle_{\gamma<\alpha}\right\rangle=t$. Also, let $\theta_{\alpha, \beta}:[0, \infty]^{\alpha} \rightarrow[0, \infty]^{\beta}$ be defined by

$$
\theta_{\alpha, \beta}\left\langle s_{\gamma}\right\rangle_{\gamma<\alpha}=\left\langle s_{\gamma}\right\rangle_{\gamma<\beta},
$$

for $1 \leq \beta<\alpha$. If $\mathbf{s} \in[0, \infty]^{\alpha}$ and $x \in[0, \infty]$, then $\mathbf{s} * x$ denoted the element in $[0, \infty]^{\alpha+1}$ defined by $\theta_{\alpha+1, \alpha}(\mathbf{s} * x)=\mathbf{s}$ and the coordinate of index $\alpha$ has value $x$. More generally, the following concatenation procedure is defined: if $\mathbf{u} \in[0, \infty]^{\alpha}$ and $\mathbf{v} \in[0, \infty]^{\beta}$, then $\mathbf{u} * \mathbf{v}$ denotes the element of $[0, \infty]^{\alpha+\beta}$ which satisfies $\theta_{\alpha+\beta, \alpha}(\mathbf{u} * \mathbf{v})=\mathbf{u}$, and for all $\gamma<\beta$, the coordinate of $\mathbf{u} * \mathbf{v}$ of index $\alpha+\gamma$ is $v_{\gamma}$. If $x \in[0, \infty]$, then $\hat{x}$ will denote the transfinite sequence, indexed by all countable ordinals, which is constantly $x$. Also let $\zeta_{\alpha}: T \times[0, \infty]^{\alpha} \rightarrow[0, \infty]^{\alpha}$ denote the projection defined by $\zeta_{\alpha}\langle t, \mathbf{s}\rangle=\mathbf{s}$, and let $\rho_{\alpha}=\theta_{\omega_{1}, \alpha}$.

Some obvious relations between the above defined functions are:

(1) $\pi_{\beta, \gamma} \circ \pi_{\alpha, \beta}=\pi_{\alpha, \gamma}$ and $\theta_{\beta, \gamma} \circ \theta_{\alpha, \beta}=\theta_{\alpha, \gamma}$ if $\gamma<\beta<\alpha$. 
(2) $\theta_{\alpha, \beta} \circ \rho_{\alpha}=\rho_{\beta}$ if $\beta<\alpha$.

(3) $\zeta_{\beta} \circ \pi_{\alpha, \beta}=\theta_{\alpha, \beta} \circ \zeta_{\alpha}$ if $\beta<\alpha$.

(4) $\pi_{\alpha, \beta}=i d \times \theta_{\alpha, \beta}$ if $\beta<\alpha$.

Definition 3.2 (Transfinite Extensions). Let $\mu \in M^{+}(T)$. Define by transfinite recursion, for each ordinal $\alpha \leq \omega_{1}$, a finite regular Borel measure $\mu_{\alpha}$ on $T \times[0, \infty]^{\alpha}$ as follows:

(1) $\mu_{0}=\mu$ and $\mu_{1}=K(\mu)$.

(2) For limit ordinals $\alpha$ : If there exists a unique measure $\nu \in M^{+}\left(T \times[0, \infty]^{\alpha}\right)$ so that

$$
\mu_{\beta}=\nu \circ \pi_{\alpha, \beta}^{-1} \text { for } \beta<\alpha,
$$

then $\mu_{\alpha}=\nu$. Otherwise set $\mu_{\alpha}=$ the zero measure in $M^{+}\left(T \times[0,1]^{\alpha}\right)$.

(3) For successor ordinals, $\alpha=\beta+1$ with $\beta>0$ : If $\mu_{\beta} \in M^{+}\left(T \times[0, \infty]^{\beta}\right)$ and there exists a unique measure $\nu \in M^{+}\left(T \times[0, \infty]^{\alpha}\right)$ so that for all $H \in$ $C\left(T \times[0, \infty]^{\alpha}\right)$

$$
\int H d \nu=\iint H(t, \mathbf{s} * x) d K\left(\mu_{\beta, \mathbf{s}}\right)(t, x) d \mu_{\beta} \circ \zeta_{\beta}^{-1}(\mathbf{s})
$$

where 's $\rightarrow \mu_{\beta, \mathbf{s}}$ ' is the factor kernel of $\mu_{\beta}$ with respect to $\zeta_{\beta}$, then $\mu_{\alpha}=\nu$. Otherwise, set $\mu_{\alpha}=$ the zero measure in $M^{+}\left(T \times[0, \infty]^{\alpha}\right)$.

The measure $\mu_{\alpha}$ is called the $\alpha^{\text {th }}$ order multifractal spectral extension measure of $\mu$, or the $\alpha^{\text {th }}$ multispectral extension of $\mu$. The measure $\sigma_{\alpha}(\mu)$ defined by $\sigma_{\alpha}(\mu)=\mu_{\alpha} \circ \zeta_{\alpha}^{-1}$ is called the $\alpha^{\text {th }}$ order multispectrum of $\mu$. For each $\alpha \leq$ $\omega_{1}$, we define the $\alpha^{\text {th }}$ order multifractal spectral extension map $(\cdot)_{\alpha}$ from $M^{+}(T)$ into $M^{+}\left(T \times[0, \infty]^{\alpha}\right)$ by $(\cdot)_{\alpha}(\mu)=\mu_{\alpha}$. Also we define the $\alpha^{\text {th }}$ order multispectrum map, $\sigma_{\alpha}$ or $\sigma_{\alpha}(\cdot)$, by $\sigma_{\alpha}(\cdot)(\mu)=\sigma_{\alpha}(\mu)$.

Theorem 3.1. For each $\mu \in M^{+}(T)$, the transfinite sequence, $\left\langle\mu_{\alpha}\right\rangle_{\alpha \leq \omega_{1}}$ is well defined with $\mu_{\alpha}$ a finite regular Borel measure on $T \times[0, \infty]^{\alpha}$, and the sequence satisfies and is uniquely determined by the three conditions:

(1) $\mu_{0}=\mu$ and $\mu_{1}=K(\mu)$.

(2) For limit ordinals $\alpha: \mu_{\alpha} \circ \pi_{\alpha, \beta}^{-1}=\mu_{\beta}$ if $\beta<\alpha$.

(3) For successor ordinals: $\alpha=\beta+1$, statement (3.2) is true for $\nu=\mu_{\alpha}$.

Further, for $1 \leq \alpha \leq \omega_{1}$,

$$
\mu_{\alpha} \circ \pi_{\alpha, \beta}^{-1}=\mu_{\beta} \text { if } \beta<\alpha,
$$

and if $1 \leq \alpha<\omega_{1}$, then there exists a ( $\mu$-essentially unique) Borel map $\phi_{\mu, \alpha}: T \rightarrow$ $[0, \infty]^{\alpha}$ so that

$$
\mu_{\alpha}=\mu \circ\left\langle i d, \phi_{\mu, \alpha}\right\rangle^{-1}
$$

If $\nu \ll \mu$, then for $1 \leq \alpha<\omega_{1}$,

$$
\phi_{\nu, \alpha}=\phi_{\mu, \alpha}, \nu \text {-a.e. }
$$

Proof. First we show that $\left\langle\mu_{\alpha}\right\rangle_{\alpha \leq \omega_{1}}$ is well defined. For any function $f$, let $\operatorname{dom}(f)$ denote the domain of $f$ and $i m(f)$ denote the set $\{f(x): x \in \operatorname{dom}(f)\}$. Let $\mathcal{M}=M^{+}(T) \cup \bigcup_{1 \leq \alpha \leq \omega_{1}} M^{+}\left(T \times[0, \infty]^{\alpha}\right)$ and $D O M=\{f: f$ is a function so that $\operatorname{dom}(f)$ is an ordinal $\leq \omega_{1}$ and $\left.i m(f) \subseteq \mathcal{M}\right\}$. By convention, DOM includes 
the empty function $\emptyset$. Let $\mathbf{0}_{\alpha}=$ the zero measure of $M^{+}\left(T \times[0, \infty]^{\alpha}\right)$. For fixed $\mu \in M^{+}(T)$, we build the obvious function Ext :DOM $\rightarrow \mathcal{M}:$ for $f=\left\langle\mu_{\beta}\right\rangle_{\beta<\alpha}$

$$
\operatorname{Ext}(f)=\left\{\begin{array}{l}
\nu, \text { if } \alpha \text { is a limit ordinal and } \nu \text { uniquely satifies (3.1). } \\
\nu, \text { if } \alpha=\beta+1 \text { and } \nu \text { uniquely satisfies (3.2). } \\
\mu, \text { if } \alpha=0 \text { (i.e., }\left\langle\mu_{\beta}\right\rangle_{\beta<\alpha}=\emptyset \text { ). } \\
\mathbf{0}_{\alpha}, \text { otherwise. }
\end{array}\right.
$$

Since the function Ext progresses through $\mathcal{M}$ for $\operatorname{dom}(f) \leq \omega_{1}$, the principle of transfinite recursion implies there exists a unique function with domain $\omega_{1}+1$ and values in $\mathcal{M}$, which we denote by $\left\langle\mu_{\alpha}\right\rangle_{\alpha \leq \omega_{1}}$, so that $\operatorname{Ext}\left(\left\langle\mu_{\beta}\right\rangle_{\beta<\alpha}\right)=\mu_{\alpha}$ for all $\alpha \leq \omega_{1}$.

Second, note statement (2.5) implies: if the function $\phi_{\mu, \alpha}$ of statement (3.4) exists at all, it is $\mu$-essentially unique.

Third, note $\operatorname{Ext}(f) \in M^{+}\left(T \times[0, \infty]^{\operatorname{dom}(f)}\right)$ for $1 \leq \operatorname{dom}(f) \leq \omega_{1}$. This implies $\mu_{\alpha}$ is a finite regular Borel measure on $T \times[0, \infty]^{\alpha}$ for $1 \leq \alpha \leq \omega_{1}$.

We now prove, by induction, the statements (3.3), (3.4), and (3.5) for the case $\alpha<\omega_{1}$ and that statement (3.2) holds with $\mu_{\alpha}=\nu$ at successor $\alpha$.

First $\mu_{0}=\mu \in M^{+}(T)$ and $\mu_{1}=K(\mu) \in M^{+}(T \times[0, \infty])$. By definition of $K, \mu=K(\mu) \circ \pi_{1,0}^{-1}$. So statement (3.3) holds for $\alpha=1$ and is vacuously true for $\alpha=0$. Further, statement (3.4) holds with $\phi_{\mu, 1}=\Phi(\mu, \cdot)$. Equation (3.5) follows from Theorem 2.1 part (3), for $\alpha=1$. Also statement (3.2) is true for $\nu=\mu_{1}$ since $\mu_{1}=K(\mu)$.

Induction hypothesis for $\alpha<\omega_{1}$ : for each $\gamma<\beta<\alpha$, the equations (3.3), (3.4), and (3.5) are true if $\alpha$ is replaced by $\beta$, and $\beta$ is replaced by $\gamma$, in those equations. Statement (3.2) holds at successor ordinals less than $\alpha$. As a further induction hypotheses, suppose that for all $\gamma<\beta<\alpha, \mu$-a.e., $\phi_{\mu, \gamma}=\theta_{\beta, \gamma} \circ \phi_{\mu, \beta}$.

Case: $\alpha$ is a limit ordinal.

The induction hypothesis implies $\left\langle\mu_{\beta}\right\rangle_{\beta \in \alpha}$ satisfy the Kolmogoroff consistency conditions. Thus there is a unique Borel measure $\nu \in M^{+}\left(T \times[0, \infty]^{\alpha}\right)$ satisfying condition (3.1), $\mu_{\alpha}=\nu$, and statement (3.3) holds.

An easy exercise will show the relations ' $\phi_{\mu, \gamma}=\theta_{\beta, \gamma} \circ \phi_{\mu, \beta}, \mu$-a.s.for $\gamma<\beta<\alpha$ ' $\mu$-uniquely defines a Borel function $\phi_{\mu, \alpha}: T \rightarrow[0, \infty]^{\alpha}$ so that for all $\beta<\alpha$, $\phi_{\mu, \beta}=\theta_{\alpha, \beta} \circ \phi_{\mu, \alpha}$. For any Borel subset, $B$, of $T \times[0, \infty]^{\beta}$,

$$
\begin{aligned}
\mu_{\alpha}\left(\pi_{\alpha, \beta}^{-1} B\right) & =\mu_{\beta}(B) \\
& =\mu \circ\left\langle i d, \phi_{\mu, \beta}\right\rangle^{-1}(B) \\
& =\mu \circ\left\langle i d,\left(\theta_{\alpha, \beta} \circ \phi_{\mu, \alpha}\right)\right\rangle^{-1}(B) \\
& =\mu \circ\left\langle i d, \phi_{\mu, \alpha}\right\rangle^{-1}\left(\pi_{\alpha, \beta}^{-1} B\right)
\end{aligned}
$$

where the first equality is due to statement (3.3) already shown for this $\alpha$, the second equality follows from statement (3.4) assumed in the induction hypothesis, and the third is the defining relation of $\phi_{\mu, \alpha}$, and the fourth is an obvious manipulation of coordinates. Since $\left\{\pi_{\alpha, \beta}^{-1}(B): \beta<\alpha\right.$ and $\left.B \in \mathcal{B}\left(T \times[0, \infty]^{\beta}\right)\right\}$ generates the Borel sets on $T \times[0, \infty]^{\alpha}$ in this limit ordinal case, statement (3.4) is true for $\alpha$. Let $\nu \ll \mu$. Since the function $\phi_{\nu, \alpha}$ is $\nu$-uniquely determined by the stability of coordinates, the $\nu$-essential equality $\phi_{\mu, \beta}=\phi_{\nu, \beta}$ for $\beta<\alpha$, implies the $\nu$-essential equality $\phi_{\mu, \alpha}=\phi_{\nu, \alpha}$.

Case: $\alpha=\beta+1$ for some $\beta$. 
By hypothesis, $\mu_{\beta} \in M^{+}\left(T \times[0, \infty]^{\beta}\right)$. The factor kernel ' $\mathrm{s} \rightarrow \mu_{\beta, \mathrm{s}}$ ' of $\mu_{\beta}$ with respect to $\zeta_{\beta}$ exists $^{1}$ and can be chosen to be weak* Borel. Since $\Phi$ is weakly Borel (Theorem 2.1), the function $K$ is weak* Borel. So, the function ' $\mathbf{s} \rightarrow K\left(\mu_{\beta, \mathbf{s}}\right)$ ' is weak* Borel with these choices and takes on values in $\operatorname{Pr}(T \times[0, \infty])$. It follows that there is a unique finite regular Borel measure, $\nu$, on $T \times[0, \infty]^{\alpha}$, so that (3.2) holds ${ }^{2}$ and $\mu_{\alpha}=\nu$. By the induction hypothesis, there is a Borel $\phi_{\mu, \beta}$ so that statement (3.4) is satisfied for $\beta$. For any $H \in C\left(T \times[0, \infty]^{\alpha}\right)$,

$$
\begin{aligned}
\int H d \mu_{\alpha} & =\iint H(t, \mathbf{u} * x) d K\left(\mu_{\beta, \mathbf{u}}\right)(t, x) d \mu_{\beta} \circ \zeta_{\beta}^{-1}(\mathbf{u}) \\
& =\iint H\left(t, \mathbf{u} * \Phi\left(\mu_{\beta, \mathbf{u}}, t\right)\right) d \mu_{\beta, \mathbf{u}}(t) d \mu_{\beta} \circ \zeta_{\beta}^{-1}(\mathbf{u})
\end{aligned}
$$

where the first equality is by definition of Ext, and the second equality is by definition of $K$. But by definition of the factor kernel ' $\mathbf{u} \rightarrow \mu_{\beta, \mathbf{u}}$ ' and by statement (3.4) for $\beta$, the last expression becomes $\int H\left(t, \phi_{\mu, \beta}(t) * \Phi\left(\mu_{\beta, \phi_{\mu, \beta}(t)}, t\right)\right) d \mu(t)$. Now, the kernel ' $\mathbf{u} \rightarrow \mu_{\beta, \mathbf{u}}$ ' can be chosen to be weak* Borel and the function $\phi_{\mu, \beta}$ is Borel, and so the function ' $t \rightarrow \Phi\left(\mu_{\beta, \phi_{\mu, \beta}(t)}, t\right)$ ' is Borel given these choices. Define $\phi_{\mu, \alpha}(t)=\phi_{\mu, \beta}(t) * \Phi\left(\mu_{\beta, \phi_{\mu, \beta}(t)}, t\right)$. By this definition, we extend the induction hypotheses by $\phi_{\mu, \gamma}=\theta_{\alpha, \gamma} \circ \phi_{\mu, \alpha}$ for $\gamma<\beta$.

If $\nu \ll \mu$, then $\nu_{\beta} \circ \zeta_{\beta}^{-1} \ll \mu_{\beta} \circ \zeta_{\beta}^{-1}$. Further, (2.4) implies: for $\nu_{\beta} \circ \zeta_{\beta}^{-1}$-a.e.-u, $\nu_{\beta, \mathbf{u}} \ll \mu_{\beta, \mathbf{u}}$. Theorem 2.1 part (3) implies $\nu_{\beta} \circ \zeta_{\beta}^{-1}$-a.e.-u, $\Phi\left(\nu_{\beta, \mathbf{u}}, \cdot\right)=\Phi\left(\mu_{\beta, \mathbf{u}}, \cdot\right)$. But by induction hypothesis, $\nu$-a.e., $\phi_{\nu, \beta}=\phi_{\mu, \beta}$. Thus, also $\nu$-a.e., $\Phi\left(\nu_{\left.\beta, \phi_{\nu, \beta}, \cdot\right)}\right)$ $\Phi\left(\mu_{\beta, \phi_{\mu, \beta}}, \cdot\right)$. The last two statements imply $\phi_{\nu, \alpha}=\phi_{\mu, \alpha} \nu$-a.e.

For the case $\omega_{1}$, note statement (3.3) being true for all $\beta<\alpha<\omega_{1}$ implies there is a unique Baire measure so that statement (3.3) is true for $\alpha=\omega_{1}$. Since the Baire measure is finite, and $T \times[0, \infty]^{\omega_{1}}$ is a compact Hausdorff, there is a unique regular Borel measure on $T \times[0, \infty]^{\omega_{1}}$ which extends this Baire measure to the Borel sets. See Theorem 7.2.8 of [2]. Statement (3.3) is still true for this Borel extension.

From what we have proven so far, conditions (1), (2), and (3) are true for the sequence $\left\langle\mu_{\alpha}\right\rangle$ and in fact the measures defined by equations (3.1) and (3.2) are unique. This implies that there can be only one transfinite sequence which satisfies (1), (2), and (3).

Definition 3.3. For $\alpha<\omega_{1}$, we call $\phi_{\mu, \alpha}$ the $\alpha^{\text {th }}$ order local dimension map.

That there is a Baire measurable function $\phi_{\mu, \omega_{1}}: T \rightarrow[0, \infty]^{\omega_{1}}$ so that the fact $\mu_{\omega_{1}}=\mu \circ\left\langle i d, \phi_{\mu, \omega_{1}}\right\rangle^{-1}$ holds for Baire sets could be proved now if we wished. Instead, we shall prove in Theorem 4.3 that there is a Borel measurable function $\phi_{\mu, \omega_{1}}$ so that $\mu_{\omega_{1}}=\mu \circ\left\langle i d, \phi_{\mu, \omega_{1}}\right\rangle^{-1}$ holds true on Borel sets. The specific form of $\phi_{\mu, \omega_{1}}$ will be pointed out there in the proof. Also a uniqueness characterization for the measures $\mu_{\alpha}$ in terms of disintegration kernels will be given later in Corollary 6.8 .

Corollary 3.2. For $1 \leq \alpha \leq \omega_{1}$, and $\mu \in M^{+}(T)$, the measures $\sigma_{\alpha}(\mu)$ is a welldefined finite regular Borel measure on $[0, \infty]^{\alpha}$ with

$$
\mu_{\alpha}\left(T \times[0, \infty]^{\alpha}\right)=\sigma_{\alpha}(\mu)\left([0, \infty]^{\alpha}\right)=\mu(T) .
$$

\footnotetext{
${ }^{1} T \times[0, \infty]^{\beta}$ is compact metric for $\beta<\omega_{1}$.

${ }^{2}$ See the first paragraph of Remark 9.3 for a note on measurability of "generalized" $K$ and $\Phi$.
} 
Further for $1 \leq \beta<\alpha \leq \omega_{1}$, the consistency condition

$$
\sigma_{\beta}(\mu)=\sigma_{\alpha}(\mu) \circ \theta_{\alpha, \beta}^{-1}
$$

holds and if $1 \leq \beta<\alpha<\omega_{1}$, for $\sigma_{\beta}(\mu)$-a.e.-s,

$$
\mu_{\beta, \mathbf{s}}=w^{*}-\int \mu_{\alpha, \mathbf{s} * \mathbf{u}} d \sigma_{\alpha}(\mu)_{\mathbf{s}}(\mathbf{u})
$$

where ' $\mathrm{s} \rightarrow \sigma_{\alpha}(\mu)_{\mathbf{s}}$ ' is the factor kernel of $\sigma_{\alpha}(\mu)$ with respect to $\theta_{\alpha, \beta}$.

Proof. Since $\mu_{\alpha}$ is finite regular Borel measure on $T \times[0, \infty]^{\alpha}$ and the function $\zeta_{\omega_{1}}$ is an open continuous map on $T \times[0, \infty]^{\alpha}, \sigma_{\alpha}(\mu)=\mu_{\alpha} \circ \zeta_{\alpha}^{-1}$ is a well-defined finite regular Borel measure on $[0, \infty]^{\alpha}$. Statement (3.6) follows from (3.3) with $\beta=0$, and the definition of $\sigma_{\alpha}(\mu)$. Since $\mu_{\alpha} \circ \pi_{\alpha, \beta}^{-1}=\mu_{\beta}$ by (3.3), and $\zeta_{\beta}=\theta_{\alpha, \beta} \circ \zeta_{\alpha}$, (3.7) follows. But for $h \in C\left(T \times[0, \infty]^{\beta}\right)$, and for $\alpha<\omega_{1}$,

$$
\begin{aligned}
\iint & h(t, \mathbf{s}) d \mu_{\beta, \mathbf{s}}(t) d \sigma_{\beta}(\mu)(\mathbf{s}) \\
= & \int h(t, \mathbf{s}) d \mu_{\beta}(t, \mathbf{s}) \\
= & \iint h\left(t, \theta_{\alpha, \beta}(\mathbf{v})\right) d \mu_{\alpha, \mathbf{v}}(t) d \sigma_{\alpha}(\mu)(\mathbf{v}) \\
& =\iiint h(t, \mathbf{s}) d \mu_{\alpha, \mathbf{s} * \mathbf{u}}(t) d \sigma_{\alpha}(\mu)_{\mathbf{s}}(\mathbf{u}) d\left(\sigma_{\alpha}(\mu) \circ \theta_{\alpha, \beta}^{-1}\right)(\mathbf{s})
\end{aligned}
$$

where the first equality follows from the definition of factor kernel ' $\mathbf{s} \rightarrow \mu_{\beta, \mathbf{s}}$ ', the second equality follows from (3.3), the third from (3.3), and the fourth by definition of factor kernel 's $\rightarrow \sigma_{\alpha}(\mu)_{\mathbf{s}}$ ' and making an obvious substitution of $\mathbf{s} * \mathbf{u}$ for $\mathbf{v}$. Thus (3.8) follows.

Note if the factor kernel is defined for $\alpha=\omega_{1}$, then (3.8) also makes sense for that $\alpha$. We shall see that $\mu_{\omega_{1}}$ has compact metrizable support and so such is the case. (See Theorem 4.3 condition (1) and Lemma 4.2.)

Corollary 3.3. For $1 \leq \alpha<\omega_{1}$, and $\mu \in M^{+}(T)$,

$$
\sigma_{\alpha}(\mu)=\mu \circ \phi_{\mu, \alpha}^{-1}
$$

and $\sigma_{\alpha}(\mu)$-a.e.-s,

$$
\mu_{\alpha, \mathbf{s}}=\mu_{\phi_{\mu, \alpha}=\mathbf{s}}
$$

where ' $\mathbf{s} \rightarrow \mu_{\alpha, \mathbf{s}}$ ' is the factor kernel of $\mu_{\alpha}$ with respect to $\zeta_{\alpha}$ and where 's $\rightarrow$ $\mu_{\phi_{\mu, \alpha}=\mathbf{s}}$ is the decomposition kernel of $\mu$ with respect to $\phi_{\mu, \alpha}$. Further if $\nu \ll \mu$, then $\sigma_{\alpha}(\nu) \ll \sigma_{\alpha}(\mu)$, and $\nu_{\alpha} \ll \mu_{\alpha}$.

Proof. By definition, (3.4), and the identity $\zeta_{\alpha} \circ\left\langle i d, \phi_{\mu, \alpha}\right\rangle=\phi_{\mu, \alpha}$,

$$
\sigma_{\alpha}(\mu)=\mu_{\alpha} \circ \zeta_{\alpha}^{-1}=\mu \circ\left\langle i d, \phi_{\mu, \alpha}\right\rangle^{-1} \circ \zeta_{\alpha}^{-1}=\mu \circ \phi_{\mu, \alpha}^{-1}
$$

And (3.10) follows from (2.6). The last statement follows since by Theorem 3.1, if $\nu \ll \mu$, then $\phi_{\nu, \alpha}=\phi_{\mu, \alpha}, \nu$-a.e.

Theorem 3.4. If $1 \leq \alpha<\omega_{1}$ and $\beta \leq \omega_{1}$, for every $H \in C\left(T \times[0, \infty]^{\alpha+\beta}\right)$,

$$
\int H d \mu_{a+\beta}=\iint H(t, \mathbf{s} * \mathbf{u}) d \mu_{\alpha, \mathbf{s} ; \beta}(t, \mathbf{u}) d \sigma_{\alpha}(\mu)(\mathbf{s})
$$


where $\mu_{\alpha, \mathbf{s} ; \beta}$ is the $\beta^{\text {th }}$-multispectral expansion of $\mu_{\alpha, \mathbf{s}}$, and $\left\langle\mu_{\alpha, \mathbf{s}}\right\rangle_{\mathbf{s} \in[0, \infty]^{\alpha}}$ is the factor kernel of $\mu_{\beta}$ with respect to $\zeta_{\beta}$.

Proof. Fix $1 \leq \alpha<\omega_{1}$. The statement is trivial for $\beta=0$. Suppose the statement is true for all $\gamma<\beta$.

Case: $\beta$ is a limit ordinal.

If $h_{\alpha+\eta} \in C\left(T \times[0, \infty]^{\alpha+\eta}\right)$ for some $\eta<\beta$, then

$$
\begin{aligned}
\int h_{\alpha+\eta} \circ \pi_{\alpha+\beta, \alpha+\eta} d \mu_{\alpha+\beta} & =\int h_{\alpha+\eta} d \mu_{\alpha+\eta} \\
& =\iint h_{\alpha+\eta}(t, \mathbf{s} * \mathbf{u}) d \mu_{\alpha, \mathbf{s} ; \eta}(t, \mathbf{u}) d \sigma_{\alpha}(\mu)(\mathbf{s})
\end{aligned}
$$

where the first equality is (3.3) and the second equality is the induction hypothesis. Using (3.3) but this time with respect to the pair $\mu_{\alpha, \mathbf{s} ; \beta}$ and $\pi_{\beta, \eta}$, and also an obvious fact about coordinates, (3.12) implies

$$
\begin{aligned}
\int h_{\alpha+\eta} \circ \pi_{\alpha+\beta, \alpha+\eta} d \mu_{\alpha+\beta} & =\iint h_{\alpha+\eta}(t, \mathbf{s} * \mathbf{u}) d \mu_{\alpha, \mathbf{s} ; \beta} \circ \pi_{\beta, \eta}^{-1}(t, \mathbf{u}) d \sigma_{\alpha}(\mu)(\mathbf{s}) \\
& =\iint h_{\alpha+\eta} \circ \pi_{\alpha+\beta, \eta}(t, \mathbf{s} * \mathbf{v}) d \mu_{\alpha, \mathbf{s} ; \beta}(t, \mathbf{v}) d \sigma_{\alpha}(\mu)(\mathbf{s}) .
\end{aligned}
$$

Since $\beta$ is a limit ordinal, the Stone-Weierstrass Theorem implies that the ring $\left\{h_{\alpha+\eta} \circ \pi_{\alpha+\beta, \alpha+\eta}: h_{\alpha+\eta} \in C\left(T \times[0, \infty]^{\alpha+\eta}\right)\right.$ and $\left.\eta<\beta\right\}$ is uniformly dense in $C\left(T \times[0, \infty]^{\alpha+\beta}\right)$. And so the case is done.

Case: $\beta=\gamma+1$.

By the induction hypothesis, for $h \in C\left(T \times[0, \infty]^{\alpha+\gamma}\right)$,

$$
\int h d \mu_{\alpha+\gamma}=\iint h(t, \mathbf{s} * \mathbf{u}) d \mu_{\alpha, s ; \gamma}(t, \mathbf{u}) d \sigma_{\alpha}(\mu)(\mathbf{s}) .
$$

Using Corollary 3.3, the above equality implies: for $g \in C\left([0, \infty]^{\alpha+\gamma}\right)$,

$$
\int g d \sigma_{\alpha+\gamma}(\mu)=\iint g(\mathbf{s} * \mathbf{u}) d \sigma_{\gamma}\left(\mu_{\alpha, s}\right)(\mathbf{u}) d \sigma_{\alpha}(\mu)(\mathbf{s}) .
$$

and $\sigma_{\alpha+\gamma}(\mu)$-a.e.-s $* \mathbf{u}$,

$$
\mu_{\alpha+\gamma, \mathbf{s} * \mathbf{u}}=\mu_{\alpha, \mathbf{s}: \gamma, \mathbf{u}} .
$$

Thus for $h \in C\left(T \times[0, \infty]^{\alpha+\beta}\right)$,

$$
\begin{aligned}
\int h d \mu_{\alpha+\beta} & =\iint h(t, \mathbf{v} * x) d K\left(\mu_{\alpha+\gamma, \mathbf{v}}\right)(t, x) d \sigma_{\alpha+\gamma}(\mu)(\mathbf{v}) \\
& =\iint h(t, \mathbf{s} * \mathbf{u} * x) d K\left(\mu_{\alpha, \mathbf{s}: \gamma, \mathbf{u}}\right)(t, x) d \sigma_{\gamma}\left(\mu_{\alpha, \mathbf{s}}\right)(\mathbf{u}) \sigma_{\alpha}(\mu)(\mathbf{s})
\end{aligned}
$$

where the first equality follows from the definition of $\mu_{\alpha+\beta}$ and the second equality follows from (3.13) and (3.14). Applying the definition of $\mu_{\alpha, \mathbf{s} ; \beta}$ for $\beta=\gamma+1$, the case is done.

Corollary 3.5. For any $\mu \in M^{+}(T)$, for any $\alpha<\omega_{1}$, for $\sigma_{\alpha}(\mu)$-a.e.-s,

$$
\sigma_{1}\left(\mu_{\alpha, \mathbf{s}}\right)=\sigma_{\alpha+1}(\mu)_{\mathbf{s}}
$$

where ' $\mathbf{s} \rightarrow \mu_{\alpha, \mathbf{s}}$ ' is the factor kernel of $\mu_{\alpha}$ with respect to $\zeta_{\alpha}$, and ' $\mathrm{s} \rightarrow \sigma_{\alpha+1}(\mu)_{\mathbf{s}}$ ' is the factor kernel of $\sigma_{\alpha+1}(\mu)$ with respect to $\theta_{\alpha+1, \alpha}$. Further, for $\sigma_{\alpha}(\mu)$-a.e.-s, 
$\sigma_{\alpha+1}(\mu)_{\mathbf{s}}-a . e .-x$,

$$
\mu_{\alpha, \mathbf{s} ; 1, x}=\mu_{\alpha+1, \mathbf{s} * x} .
$$

Proof. This follows easily from (3.11) with $\beta=1$.

Remember if $x \in[0, \infty]$, then $\hat{x}$ is the element of $[0, \infty]^{\omega_{1}}$ which is constantly $x$ in every coordinate.

Lemma 3.6. A measure $\mu \in M^{+}(T)$ is unidimensional of dimension $x$ if and only if $\mu_{\omega_{1}}=\mu \times \delta_{\widehat{x}}$.

Proof. Suppose $\mu$ is unidimensional of dimension $x$. Then $\mu_{1}=\mu \times \delta_{x}$ by definition of unidimensionality. Suppose the induction hypothesis $\mu_{\beta}=\mu \times\left(\delta_{x}\right)^{\beta}$ for $1 \leq$ $\beta<\alpha$. If $\alpha$ is a limit ordinal then (3.3) and the uniqueness part of Kolmogoroff extension theorem implies $\mu_{\alpha}=\mu \times\left(\delta_{x}\right)^{\alpha}$. If $\alpha=\beta+1$, then for $h \in C\left(T \times[0, \infty]^{\alpha}\right)$,

$$
\int h d \mu_{\alpha}=\iint h(t, \mathbf{s} * y) d K(\mu)(t, y) d\left(\delta_{x}\right)^{\beta}(\mathbf{s})
$$

since $\mu_{\beta}=\mu \times\left(\delta_{x}\right)^{\beta}$. Since $K(\mu)=\mu \times \delta_{x}$, necessity follows.

Suppose $\mu_{\omega_{1}}=\mu \times \delta_{\widehat{x}}$. (3.3) implies the converse.

\section{First Version of Necessity}

Before stating the first version of necessity, the following lemmas should be noted:

Lemma 4.1. Let $F$ be a closed subset of $[0, \infty]^{\omega_{1}}$, for each $\alpha<\omega_{1}$, let $F_{\alpha}=$ $\rho_{\alpha}^{-1}\left(\rho_{\alpha}(F)\right)$, and let $\kappa$ be a finite regular Borel measure on $[0, \infty]^{\omega_{1}}$. Then

$$
\lim _{\alpha \rightarrow \omega_{1}} \kappa\left(F_{\alpha}\right)=\kappa(F) .
$$

In particular, if $\kappa$ is supported on $F_{\alpha}$ for each $\alpha<\omega_{1}$, then $\kappa$ is supported on $F$.

Proof. Let $\epsilon>0$. Since $\kappa$ is regular, there is an open subset, $O$, of $[0, \infty]^{\omega_{1}}$ which is a superset of $F$ and $\kappa(O) \leq \kappa(F)+\epsilon$. Since $F$ is compact, there is a finite union of open cylinders, $O^{\prime}$, so that $F \subseteq O^{\prime} \subseteq O$. Since $O^{\prime}$ is a finite union of open cylinders, there is a countable ordinal $\gamma$, so that for any $\beta \geq \gamma, \rho_{\beta}^{-1}\left(\rho_{\beta}\left(O^{\prime}\right)\right)=O^{\prime}$. Thus,

$$
\kappa(F) \leq \kappa\left(F_{\beta}\right) \leq \kappa\left(O^{\prime}\right) \leq \kappa(O) \leq \kappa(F)+\epsilon \text { for } \beta \geq \gamma .
$$

Remark 4.1. Lemma 4.1 is not true if the word 'closed' is replaced by the word 'open'.

If $\alpha<\omega_{1}$, then let $\Delta_{\alpha}$ denote the set $\left\{\mathbf{s} \in[0, \infty]^{\omega_{1}}: \forall \gamma \forall \beta\right.$ if $\gamma<\beta$, then $s_{\gamma} \geq s_{\beta}$ and $\forall \delta$ if $\delta \geq \alpha$, then $\left.s_{\alpha}=s_{\delta}\right\}$. Easily, $\Delta_{\alpha}$ is a compact metrizable subset of $[0, \infty]^{\omega_{1}}$. If $\kappa$ is a finite regular Borel measure on $[0, \infty]^{\omega_{1}}$ which is supported on $\Delta_{\alpha}$, then the decomposition kernel of $\kappa$ with respect to $\rho_{\beta}$ is well-defined. Let $\Delta_{\omega_{1}}$ denote the set $\left\{\mathbf{s} \in[0, \infty]^{\omega_{1}}: \forall \gamma \forall \beta\right.$ if $\gamma<\beta<\omega_{1}$, then $\left.s_{\gamma} \geq s_{\beta}\right\}$. Since decreasing sequences in $[0, \infty]$ which are indexed by elements of $\omega_{1}$ are eventually constant, $\Delta_{\omega_{1}}=\bigcup_{\alpha<\omega_{1}} \Delta_{\alpha}$. The set $\Delta_{\omega_{1}}$ is a compact subset of $[0, \infty]^{\omega_{1}}$, however, unlike $\Delta_{\alpha}, \Delta_{\omega_{1}}$ is not metrizable.

Lemma 4.2. If $\kappa$ is a finite regular Borel measure on $[0, \infty]^{\omega_{1}}$, then the following three statements are equivalent:

(1) For all $\alpha<\beta<\omega_{1}, \kappa$ is supported on the set $\left\{\mathbf{s} \in[0, \infty]^{\omega_{1}}: s_{\alpha} \geq s_{\beta}\right\}$. 
(2) $\kappa$ is supported on $\Delta_{\omega_{1}}$.

(3) There is a $\alpha<\omega_{1}$ so that $\kappa$ is supported on $\Delta_{\alpha}$.

Proof. We will show (1) implies (2) implies (3) implies (1).

Suppose (1). Since $\kappa$ is countably additive, for any countable ordinal $\alpha$, (1) implies $\kappa$ is supported on the set $A_{\alpha}=\left\{\mathbf{s} \in[0, \infty]^{\alpha}: s_{\gamma} \geq s_{\beta}\right.$ if $\left.\gamma \leq \beta<\alpha\right\}$. But $\rho_{a}^{-1} \rho_{a}\left(\Delta_{\omega_{1}}\right)=A_{\alpha}$. By Lemma 4.1, $\kappa$ is supported on $\Delta_{\omega_{1}}$. Thus (2) follows.

Suppose (2). For each $\alpha<\omega_{1}$, let $\varphi_{\alpha}(x)=\kappa\left(\left\{\mathbf{s} \in[0, \infty]^{\omega_{1}}: s_{\alpha} \leq x\right\}\right)$. Since $\kappa\left(\Delta_{\omega_{1}}\right)=\kappa\left([0, \infty]^{\omega_{1}}\right)$, for each $x,\left\langle\varphi_{\alpha}(x)\right\rangle_{\alpha<\omega_{1}}$ is non-decreasing in $[0, \infty)$ and so eventually constant. Let $D$ be a countable dense set of $[0, \infty]$, which contains $\{0, \infty\}$. There is a countable ordinal $\delta$ so that for each $x \in D,\left\langle\varphi_{\alpha}(x)\right\rangle_{\delta \leq \alpha<\omega_{1}}$ is a constant sequence. By the right continuity of the $\varphi_{\alpha}$ 's,

$$
\text { for all } \gamma \geq \delta, \varphi_{\gamma}=\varphi_{\delta} \text {. }
$$

Let $B_{\gamma}=\left\{\mathbf{s} \in[0, \infty]^{\omega_{1}}: s_{\gamma}=s_{\delta}\right\}$. (2) implies

$$
\text { for } \gamma \geq \delta, \kappa \text {-a.e., } s_{\delta}-s_{\gamma} \geq 0 \text {, }
$$

(where $\infty-\infty=0$ conventionally). Statements (4.4) and (4.3) imply for $\gamma \geq \delta$, $\kappa\left(B_{\gamma}\right)=\kappa\left([0, \infty]^{\omega_{1}}\right)$. Thus for any countable $\beta \geq \delta, \kappa$ is supported on $\bigcap_{\delta \leq \gamma \leq \beta} B_{\gamma}$. By Lemma 4.1,

$$
\kappa \text { is supported on } \bigcap_{\delta \leq \gamma<\omega_{1}} B_{\gamma} .
$$

Statement (4.5) and $\kappa\left(\Delta_{\omega_{1}}\right)=\kappa\left([0, \infty]^{\omega_{1}}\right)$ imply (3).

Obviously, (3) implies (1).

Theorem 4.3. For each $\mu \in M^{+}(T)$, the following two conditions are satisfied:

(1) For all $\alpha<\beta<\omega_{1}, \sigma_{\omega_{1}}(\mu)$-a.e.-s,

$$
s_{\alpha} \geq s_{\beta}
$$

where $\mathbf{s}=\left\langle s_{\gamma}\right\rangle_{\gamma \in \omega_{1}}$.

(2) For each countable ordinal $\beta$, for $\sigma_{\beta}(\mu)$-a.e.-s, for every $x \in[0, \infty]$, if $\sigma_{\beta+1}(\mu)_{\mathbf{s}}(\{x\})>0$, then

$$
\sigma_{\omega_{1}}(\mu)_{\rho_{\beta+1}=\mathbf{s} * x}=\delta_{\mathbf{s} * \widehat{x}}
$$

where $\left\langle\sigma_{\beta+1}(\mu)_{\mathbf{s}}\right\rangle_{\mathbf{s} \in[0, \infty]^{\beta}}$ is the factor kernel of $\sigma_{\beta+1}(\mu)$ with respect to $\theta_{\beta+1, \beta}$ and $\left\langle\sigma_{\omega_{1}}(\mu)_{\rho_{\beta+1}=\mathbf{s} * x}\right\rangle_{\mathbf{s} * x \in[0, \infty]^{\beta+1}}$ is the decomposition kernel of $\sigma_{\omega_{1}}(\mu)$ with respect to $\rho_{\beta+1}$.

Further, there exists a Borel function $\phi_{\mu, \omega_{1}}: T \rightarrow[0, \infty]^{\omega_{1}}$ so that

$$
\mu_{\omega_{1}}=\mu \circ\left\langle i d, \phi_{\mu, \omega_{1}}\right\rangle^{-1} \text {. }
$$

If $\nu \in M^{+}(T)$ and $\nu \ll \mu$, then $\nu$-a.e.,

$$
\phi_{\nu, \omega_{1}}=\phi_{\mu, \omega_{1}} .
$$

Proof. Let $m=\mu(T)$. For each $\alpha<\omega_{1}$, let $A_{\alpha}=\left\{\mathbf{s} \in[0, \infty]^{\alpha}: s_{\gamma} \geq s_{\beta}\right.$ if $\gamma \leq \beta<\alpha\}$. It will be shown by induction that for $1 \leq \alpha<\omega_{1}$, the following is true:

$S(\alpha): \sigma_{\alpha}(\mu)\left(A_{\alpha}\right)=m$, and $\sigma_{\alpha}(\mu)$-a.e.-s, $\mu_{\alpha, \mathbf{s}}$ is supported on a set of dimension $\leq\left(\inf _{\gamma<\alpha} s_{\gamma}\right)$. 
For $\alpha=1, A_{1}=[0, \infty]$. So trivially $\sigma_{1}(\mu)\left(A_{1}\right)=m$. The statement ' $\sigma_{1}(\mu)$-a.e.$s_{0}, \mu_{1, s_{0}}$ is supported on a set of dimension $\leq s_{0}$ ' follows from Theorem 2.3 part (1).

Induction hypothesis: Suppose $S(\beta)$ is true for $1 \leq \beta<\alpha$.

Case: $\alpha$ is a countable limit ordinal.

Let $\left\langle\gamma_{i}\right\rangle_{i=1}^{\infty}$ be a sequence of ordinals converging up to $\alpha$. Then $\theta_{\alpha, \gamma_{i}}^{-1}\left(A_{\gamma_{i}}\right)$ converges downward to $A_{\alpha}$ and

$$
\sigma_{\alpha}(\mu)\left(A_{\alpha}\right)=\lim _{i \rightarrow \infty} \sigma_{\alpha}(\mu)\left(\theta_{\alpha, \gamma_{i}}^{-1}\left(A_{\gamma_{i}}\right)\right)
$$

by the bounded convergence theorem. But $\sigma_{\alpha}(\mu)\left(\theta_{\alpha, \gamma_{i}}^{-1}\left(A_{\gamma_{i}}\right)\right)=\sigma_{\gamma_{i}}(\mu)\left(A_{\gamma_{i}}\right)=m$ by (3.7) and the induction hypothesis. Thus $\sigma_{\alpha}(\mu)\left(A_{\alpha}\right)=m$.

Let $\beta<\alpha$, by the induction hypothesis $\sigma_{\beta}(\mu)$-a.e.-s, $\mu_{\beta, \mathbf{s}}$ is supported on a set of dimension $\leq \inf _{\gamma<\beta} s_{\gamma}$. From this last and (3.8), for $\sigma_{\beta}(\mu)$-a.e.-s, $\sigma_{\alpha}(\mu)_{\mathbf{s}^{-}}$ a.e.-u, $\mu_{\alpha, \mathbf{s} * \mathbf{u}}$ is supported on a set of dimension $\leq \inf _{\gamma<\beta} s_{\gamma}$. But this implies for every $\beta<\alpha, \sigma_{\alpha}(\mu)$-a.e.- $\left\langle s_{\gamma}\right\rangle_{\gamma<\alpha}, \mu_{\alpha, \mathbf{s}}$ is supported on a set of dimension $\leq$ $\inf _{\gamma<\beta} s_{\gamma}$. Thus for $\sigma_{\alpha}(\mu)$-a.e.- $\left\langle s_{\gamma}\right\rangle_{\gamma<\alpha}, \mu_{\alpha, \mathbf{s}}$ is supported on a set of dimension $\leq \inf _{i}\left(\inf _{\gamma<\gamma_{i}} s_{\gamma}\right)=\inf _{\beta<\alpha} s_{\beta} . S(\alpha)$ follows.

Case: $\alpha=\beta+1$.

By the induction hypothesis, $\sigma_{\beta}(\mu)$-a.e.-s, $\mu_{\beta, \mathbf{s}}$ is supported on a set of dimension $\leq\left(\inf _{\gamma<\beta} s_{\gamma}\right)$ and $\sigma_{\beta}(\mu)\left(A_{\beta}\right)=m$. This and Theorem 2.3 part (3) together imply: for $\sigma_{\beta}(\mu)$-a.e.-s, $\sigma_{1}\left(\mu_{\beta, \mathbf{s}}\right)$-a.e.- $x, x \leq\left(\inf _{\gamma<\beta} s_{\gamma}\right)$. By Theorem 2.3 part (1), $\sigma_{\beta}(\mu)$-a.e.-s, $\sigma_{1}\left(\mu_{\beta, \mathbf{s}}\right)$-a.e.- $x, \mu_{\beta, \mathbf{s} ; 1, x}$ is supported on a set of dimension $\leq x$. Using Theorem 3.4, the last two statements imply $\sigma_{\beta+1}$-a.e.-v, $\mu_{\beta+1, \mathbf{v}}$ is supported on a set of dimension $v_{\beta}=\inf _{\gamma<\beta+1} v_{\gamma} . S(\alpha)$ follows.

Obviously $S(\alpha)$ being true for all $\alpha<\omega_{1}$ implies condition (1). Note condition (1) and Lemma 4.2 together imply $\sigma_{\omega_{1}}(\mu)$ is supported on the compact metrizable subset $\Delta_{\alpha_{0}}$ for some countable $\alpha_{0}$. Thus for any $\beta<\omega_{1}$, the decomposition kernel $\left\langle\sigma_{\omega_{1}}(\mu)_{\rho_{\beta}=\mathbf{s}}\right\rangle_{\mathbf{s} \in[0, \infty]^{\beta}}$ is well defined.

In order to prove condition (2), fix a countable ordinal $\alpha$. By Corollary 3.5, for $\sigma_{\alpha}(\mu)$-a.e.-s,

$$
\sigma_{1}\left(\mu_{\alpha, \mathbf{s}}\right)=\sigma_{\alpha+1}(\mu)_{\mathbf{s}}
$$

where $\left\langle\mu_{\alpha, \mathbf{s}}\right\rangle_{\mathbf{s} \in[0, \infty]^{\alpha}}$ is the factor kernel of $\mu_{\alpha}$ with respect to $\zeta_{\alpha}$, and where $\left\langle\sigma_{\alpha+1}(\mu)_{\mathbf{s}}\right\rangle_{\mathbf{s} \in[0, \infty]^{\alpha}}$ is the factor kernel of $\sigma_{\alpha+1}(\mu)$ with respect to $\theta_{\alpha+1, \alpha}$. Further, for $\sigma_{\alpha}(\mu)$-a.e.-s, $\sigma_{\alpha+1}(\mu)_{\mathbf{s}}$-a.e.- $x$,

$$
\mu_{\alpha, \mathbf{s} ; 1, x}=\mu_{\alpha+1, \mathbf{s} * x}
$$

Thus by Theorem 2.3 part (2), 'for $\sigma_{\alpha}(\mu)$-a.e.-s, $\sigma_{\alpha+1}(\mu)_{\mathbf{s}}$-a.e.- $x$, if $\sigma_{\alpha+1}(\mu)_{\mathbf{s}}(\{x\})>$ 0 , then $\mu_{\alpha, \mathbf{s} ; 1, x}$ (and therefore $\mu_{\alpha+1, \mathbf{s} * x}$ ) is unidimensional of dimension $x$ '. The quantification ' $\sigma_{\alpha+1}(\mu)_{\mathbf{s}}$-a.e.- $x$, if $\sigma_{\alpha+1}(\mu)_{\mathbf{s}}(\{x\})>0$, then $\ldots$ '. 'is equivalent to the quantification 'for all $x \in[0, \infty]$, if $\sigma_{\alpha+1}(\mu)_{\mathbf{s}}(\{x\})>0$, then $\cdots$ ' since ' $\sigma_{\alpha+1}(\mu)_{\mathbf{s}}(\{x\})$ $>0$ ' implies $x$ is in the essential support of $\sigma_{\alpha+1}(\mu)_{\mathbf{s}}$. It follows from Lemma 3.6 that for $\sigma_{\alpha}(\mu)$-a.e.-s, for every $x \in[0, \infty]$, if $\sigma_{\alpha+1}(\mu)_{\mathbf{s}}(\{x\})>0$, then $\sigma_{\omega_{1}}\left(\mu_{\alpha+1, \mathbf{s} * x ; \omega_{1}}\right)$ $=\delta_{\widehat{x}}$. Since Theorem 3.4 and the last statement implies

$$
\sigma_{\omega_{1}}(\mu)_{\mathbf{s} * x}=\delta_{\mathbf{s} * x} \times \sigma_{\omega_{1}}\left(\mu_{\alpha+1, \mathbf{s} * x}\right)=\delta_{\mathbf{s} * x} \times \delta_{\widehat{x}},
$$

condition (2) is proven.

In order to show (4.6), let $\alpha_{0}$ be a countable ordinal so that $\sigma_{\omega_{1}}(\mu)$ is supported on $\Delta_{\alpha_{0}}$. Let $g_{\alpha_{0}}:[0, \infty]^{\alpha_{0}+1} \rightarrow[0, \infty]^{\omega_{1}}$ be the function defined by $g_{\alpha_{0}}(\mathbf{s})=\mathbf{s} * \hat{s}_{\alpha_{0}}$ 
where $s_{\alpha_{0}}$ is the coordinate of $\mathbf{s}$ with index $\alpha_{0}$. Easily, for $\sigma_{\alpha_{0}+1}(\mu)$-a.e.-s,

$$
\sigma_{\alpha_{0}+2}(\mu)_{\mathbf{s}}=\delta_{s_{\alpha_{0}}}
$$

since $\sigma_{\omega_{1}}(\mu)$ is supported on $\Delta_{\alpha_{0}}$. But (4.8) and Corollary 3.5 together imply: for $\sigma_{\alpha_{0}+1}(\mu)$-a.e.-s, $\sigma_{1}\left(\mu_{\alpha_{0}+1, \mathbf{s}}\right)=\sigma_{\alpha_{0}+2}(\mu)_{\mathbf{s}}=\delta_{s_{\alpha_{0}}}$. Thus $\mu_{\alpha_{0}+1, \mathbf{s}}$ is unidimensional of dimension $s_{\alpha_{0}}$, and by Lemma 3.6

$$
\mu_{\alpha_{0}+1, \mathbf{s} ; \omega_{1}}=\mu_{\alpha_{0}+1, \mathbf{s}} \times \delta_{\widehat{s}_{\alpha_{0}}}
$$

Let $\phi_{\mu, \omega_{1}}=g_{\alpha_{0}} \circ \phi_{\mu, \alpha_{o}+1}$. If $h \in C\left(T \times[0, \infty]^{\omega_{1}}\right)$, then

$$
\begin{aligned}
\int h \circ\left\langle i d, \phi_{\mu, \omega_{1}}\right\rangle(t) d \mu(t) & =\int h\left(t, g_{\alpha_{0}} \circ \phi_{\mu, \alpha_{o}+1}(t)\right) d \mu(t) \\
& =\int h\left(t, g_{\alpha_{0}}(\mathbf{s})\right) d \mu_{\alpha_{0}+1}(t, \mathbf{s}) \\
& =\iint h\left(t, \mathbf{s} * \hat{s}_{\alpha_{0}}\right) d \mu_{\alpha_{0}+1, \mathbf{s}}(t) d \sigma_{\alpha_{0}+1}(\mu)(\mathbf{s}) \\
& =\iint h(t, \mathbf{s} * \mathbf{u}) d \mu_{\alpha_{0}+1, \mathbf{s} ; \omega_{1}}(t, \mathbf{u}) d \sigma_{\alpha_{0}+1}(\mu)(\mathbf{s}) \\
& =\int h d \mu_{\omega_{1}}
\end{aligned}
$$

where the first equality follows from the definition of $\left\langle i d, \phi_{\mu, \omega_{1}}\right\rangle$, the second from (3.4), the third by the definitions of factor kernel ' $s \rightarrow \mu_{\alpha_{0}+1, \mathbf{s}}$ ' and the definition of $g_{\alpha}$, the fourth by (4.9), and the fifth by Theorem 3.4. Thus, this $\phi_{\mu, \omega_{1}}$ satisfies (4.6). Since $\sigma_{\omega_{1}}(\mu)$ is supported on a compact metrizable set, by $(2.5), \phi_{\mu, \omega_{1}}$ is essentially unique.

For (4.7), let $\nu \ll \mu$. Let $\alpha_{0}$ be a countable ordinal so that $\sigma_{\omega_{1}}(\mu)$ is supported on $\Delta_{\alpha_{0}}$. By $(3.7), \sigma_{\beta}(\mu)$ is supported on $\rho_{\beta}\left(\Delta_{\alpha_{0}}\right)$, and so by the last statement of Corollary 3.3, $\sigma_{\beta}(\nu)$ is supported on $\rho_{\beta}\left(\Delta_{\alpha_{0}}\right)$; that is, $\sigma_{\beta}(\nu)\left(\rho_{\beta}\left(\Delta_{\alpha_{0}}\right)\right)=\nu(T)$. By Lemma $4.1 \sigma_{\omega_{1}}(\nu)$ is also supported on $\Delta_{\alpha_{0}}$. By definition $\phi_{\nu, \omega_{1}}=g_{\alpha_{0}} \circ \phi_{\nu, \alpha_{o}+1}$ and since $\nu$-a.e., $\phi_{\nu, \alpha_{o}+1}=\phi_{\mu, \alpha_{o}+1}$, it follows that $\phi_{\nu, \omega_{1}}=\phi_{\mu, \omega_{1}} \nu$-a.e.

\section{Unidimensionality and the Second Version of Necessity}

Theorem 4.3 indicates among other things that in the decompositions there is a level by which unidimensionality is reached, and if unidimensionality is reached it is conserved.

Definition 5.1. Let $\kappa$ be a regular Borel probability measure on $[0, \infty]^{\omega_{1}}$. For each $\gamma<\omega_{1}$, let $\kappa_{\gamma}=\kappa \circ \rho_{\gamma}^{-1}$ and let $\left\langle\kappa_{\gamma+1, \mathbf{s}}\right\rangle_{s \in[0, \infty]^{\gamma}}$ be the factor kernel of $\kappa_{\gamma+1}$ with respect to $\theta_{\gamma+1, \gamma}$. The measure $\kappa$ is said to be a 0 -order basic multispectrum if there is a $x \in[0, \infty]$ so that $\kappa=\delta_{\widehat{x}}$. For $1 \leq \alpha<\omega_{1}, \kappa$ is said to be an $\alpha$-order basic multispectrum if

(1) $\kappa$ is supported on $\Delta_{\alpha}$,

(2) for each $\gamma<\alpha, \kappa_{\gamma}$-a.e.-s, $\kappa_{\gamma+1, \mathbf{s}}$ is non-atomic, and

(3) there exists a $\kappa_{\alpha}$-essentially defined Borel function $U$ from $[0, \infty]^{\alpha}$ into $[0, \infty]$ so that $\kappa_{\alpha}$-a.e.-s, $\kappa_{\rho_{\alpha}=\mathbf{s}}=\delta_{\mathbf{s} * \widehat{U(s)}^{3}}^{3}$.

\footnotetext{
${ }^{3}$ The decomposition kernel $\left\langle\kappa_{\rho_{\alpha}=\mathbf{s}}\right\rangle_{\mathbf{s} \in[0, \infty]^{\alpha}}$ exists since (1) is true. Also, this implies $\kappa_{\alpha}$-a.e.-s, $U(\mathbf{s}) \leq \inf _{\gamma: \gamma<\alpha} s_{\gamma}$.
} 
In this case, the measure $\kappa_{\alpha}$ is called the dimension spreading measure or the dimension diffusing measure and the Borel map $U$ is called the unidimensional map of $\kappa$. If $\mu \in M^{+}(T)$, and $\sigma_{\omega_{1}}(\mu)$ is a basic multispectrum of order $\alpha$, then $\mu$ is said to have simple $\alpha$-order dimension spreading or diffuseness and to have simple $\alpha+1$ order unidimensionality.

There has already been work done on producing measures with simple 1st order diffuseness. Some results can be seen in the examples of [21] in the case of cascades with exchangeable generators. Kahane and Katznelson have various examples of measures with simple 1st order diffuseness for special unidimensional functions $U$. See [10]. We will see that all orders of dimension spreading occur (see Section 9). Simple 0-order spreading is just unidimensionality.

Lemma 5.1. If $\kappa$ is a finite regular Borel measure on $[0, \infty]^{\omega_{1}}$ which satisfies conditions (1) and (2) of Theorem 4.3, then there exists a sequence of non-negative real numbers $\left\langle a_{i}\right\rangle_{i}$ and a sequence of basic multispectrums, $\left\langle\kappa_{i}\right\rangle_{i}$, so that $\sum_{i} a_{i}<\infty$ and $\kappa=\sum_{i} a_{i} \kappa_{i}$.

Proof. For each countable ordinal $\beta$, let $\kappa_{\beta}=\kappa \circ \rho_{\beta}^{-1}$. Condition (1) of Theorem 4.3 implies that there is a countable ordinal $\alpha$ so that $\kappa$ is supported on the compact metrizable set $\Delta_{\alpha}$. (See Lemma 4.2.) Easily,

$$
\kappa_{\alpha+1} \text {-a.e.-s, } \kappa_{\alpha+2, \mathbf{s}}\left\{s_{\alpha}\right\}=1,
$$

where "s $\rightarrow \kappa_{\alpha+2, \mathbf{s}}$ " is the factor kernel of $\kappa_{\alpha+2}$ with respect to $\theta_{\alpha+2, \alpha+1}$. It follows for $\kappa$-a.e.-s, there is a first ordinal $\gamma(\mathbf{s}) \leq \alpha+1$ so that

$$
\kappa_{\gamma(\mathbf{s})+1,\left.\mathbf{s}\right|_{\gamma(\mathbf{s})}}\left(\left\{s_{\gamma(\mathbf{s})}\right\}\right)>0 .
$$

$\left(\kappa_{1, \emptyset}=\kappa_{1}\right.$ conventionally.) By condition (2) of Theorem 4.3, $\kappa$-a.e.-s,

$$
\mathbf{s}=\left.\mathbf{s}\right|_{\gamma(\mathbf{s})} * \widehat{s_{\gamma(\mathbf{s})}} \text {. }
$$

Define $F_{\gamma}=\left\{\mathbf{s} \in \Delta_{\alpha}: \gamma(\mathbf{s})=\gamma\right\}$. Since a finite measure can have only countably many atoms, statement (5.1) implies for $\kappa_{\gamma}$-a.e.-u, there are only countably many $x$ in the real numbers so that $\mathbf{u} * \hat{x} \in F_{\gamma}$. It follows from a theorem of Lusin and Novikov that there exists countably many disjoint Borel functions $\left\{U_{\gamma, i}\right\}_{i \in \mathbb{N}}$ from subsets of $[0, \infty]^{\gamma}$ into the reals so that the set $\left\{\mathbf{u} * \widehat{U_{\gamma, i}(\mathbf{u})}: i \in \mathbb{N}\right.$, $\mathbf{u}$ is in the domain of $\left.U_{\gamma, i}\right\}$ is $\kappa$-almost equal to $F_{\gamma}$. Let $F_{\gamma, i}=\left\{\mathbf{u} * \widehat{U_{\gamma, i}(\mathbf{u}}\right) \in \Delta_{\alpha}: \mathbf{u}$ is in the domain of $\left.U_{\gamma, i}\right\}$, and $a_{\gamma, i}=\kappa\left(F_{\gamma, i}\right)$. Easily, $F_{\gamma, i}$ is a subset of $\Delta_{\gamma}$, and $\sum_{\gamma \leq \alpha+1} \sum_{i} a_{\gamma, i}=\kappa\left([0, \infty]^{\omega_{1}}\right)$. Define $\kappa_{\gamma, i}=a_{\gamma, i}^{-1} \kappa\left(F_{\gamma, i} \cap \cdot\right)$ if $a_{\gamma, i} \neq 0 .{ }^{4}$ Then $\kappa=\sum_{(\gamma, i): a_{\gamma, i} \neq 0} a_{\gamma, i} \kappa_{\gamma, i}$. Without loss of generality suppose $a_{\gamma, i} \neq 0$ for all $(\gamma, i)$.

Now we show that $\kappa_{\gamma, i}$ satisfies conditions (1), (2), and (3) of Definition 5.1. Clearly, if $\mathbf{u} \in[0, \infty]^{\gamma}, \mathbf{s}$ is in $\Delta_{\alpha}$, and $\mathbf{s}=\mathbf{u} * \widehat{x}$ for some $x \in \mathbb{R}$, then $\mathbf{s}$ is in $\Delta_{\gamma}$. Therefore, $F_{\gamma, i} \subseteq \Delta_{\gamma}, \kappa_{\gamma, i}$ is a probability measure which is supported on $\Delta_{\gamma}$, and (1) is true for the parameter $\gamma$. Let $\kappa_{\gamma, i, \beta}=\kappa_{\gamma, i} \circ \rho_{\beta}^{-1}$ for each $\beta<\omega_{1}$. Suppose $\beta<\gamma$. Now, $\kappa_{\gamma, i}$ is absolutely continuous with respect to $\kappa$, and so $\kappa_{\gamma, i, \beta}$ is absolutely continuous with respect to $\kappa_{\beta}$, and for $\kappa_{\gamma, i, \beta}$-a.e.-v, $\kappa_{\gamma, i, \beta+1, \mathbf{v}}$ is absolutely continuous with respect to $\kappa_{\beta+1, \mathbf{v}}$. See (2.4). Let $A=\{\mathbf{s} \in$ $F_{\gamma, i}: \kappa_{\gamma, i, \beta+1,\left.\mathbf{s}\right|_{\beta}}$ is absolutely continuous with respect to $\kappa_{\beta+1,\left.\mathbf{s}\right|_{\beta}}$ and $\kappa_{\gamma, i, \beta+1,\left.\mathbf{s}\right|_{\beta}}$ is supported on $\left\{x \in \mathbb{R}: \exists \mathbf{u} \in F_{\gamma, i}\right.$ s.t. $\left.\mathbf{s}\right|_{\beta}=\left.\mathbf{u}\right|_{\beta}$ and $\left.\left.x=u_{\beta}\right\}\right\}$. Clearly $\kappa_{\gamma, i}(A)=1$. If $\mathbf{s} \in A$ and $\kappa_{\gamma, i, \beta+1,\left.\mathbf{s}\right|_{\beta}}$ is not non-atomic, then there exists an $\mathbf{u} \in F_{\gamma, i}$ so that

\footnotetext{
${ }^{4}$ where $\kappa\left(F_{\gamma, i} \cap \cdot\right)(A)=\kappa\left(F_{\gamma, i} \cap A\right)$.
} 
$\left.\mathbf{s}\right|_{\beta}=\left.\mathbf{u}\right|_{\beta}$ and $\kappa_{\gamma, i, \beta+1,\left.\mathbf{s}\right|_{\beta}}\left(\left\{u_{\beta}\right\}\right)>0$. Thus, $\kappa_{\beta+1,\left.\mathbf{s}\right|_{\beta}}\left(\left\{u_{\beta}\right\}\right)>0$,. This implies $\gamma(\mathbf{u}) \leq \beta<\gamma$ and so $\mathbf{u} \notin F_{\gamma, i}$. Contradiction. Thus for $\beta<\gamma$, for $\kappa_{\gamma, i}$-a.e.$\mathbf{s}, \kappa_{\gamma, i, \beta+1,\left.\mathbf{s}\right|_{\beta}}$ is non-atomic, implying (2) is true for the parameter $\gamma$. Since $\kappa_{\gamma, i}$ is supported on $F_{\gamma, i},(3)$ is true for $U=U_{\gamma, i}$.

The converse of Lemma 5.1 is true also. See Remark 9.1. Putting Lemma 5.1 and Theorem 4.3 together implies the following second version of necessity:

Corollary 5.2 (Second Version of Necessity). If $\mu \in M^{+}(T)$, then there is a sequence of non-negative real numbers $\left\langle a_{i}\right\rangle$ and a sequence of basic multispectrums, $\left\langle\kappa_{i}\right\rangle_{i}$, so that $\sum_{i} a_{i}<\infty$ and $\sigma_{\omega_{1}}(\mu)=\sum_{i} a_{i} \kappa_{i}$.

\section{Some Linearity Results and A Uniqueness Characterization}

Before proceeding to the proof of sufficiency, some linearity results will be shown.

Definition 6.1. The set $M^{+}(X)$ is a cone in the sense that if $\mu, \nu \in M^{+}(X)$ and $a, b \in[0, \infty)$, then $a \mu+b \nu \in M^{+}(X)$ and $M^{+}(X) \cap-M^{+}(X)=\emptyset$. A subset $\mathcal{S}$ of $M^{+}(X)$ is called a subcone if for every $\mu, \nu \in M^{+}(X)$ and every $a, b \in[0, \infty)$, we have $a \mu+b \nu \in M^{+}(X)$.

The following is an easy linearity result. In the case $\alpha=1$, it has already been noted by Cutler.

Theorem 6.1. If $1 \leq \alpha \leq \omega_{1}$, then $(\cdot)_{\alpha}$ and $\sigma_{\alpha}$ are linear and strongly continuous.

Theorem 6.1 is a consequence of the following lemma:

Lemma 6.2. Let $T$ be a compact metric space, and $(X, \Sigma)$ is a measurable space. If for each $\mu \in M^{+}(T)$, a measurable function $\phi_{\mu}: T \rightarrow X$ is defined so that the collection $\left\{\phi_{\mu}\right\}_{\mu \in M^{+}(T)}$ satisfies

$$
\text { 'if } \nu \ll \mu \text {, then } \nu \text {-a.e. } \phi_{\nu}=\phi_{\mu} \text { ' }
$$

then the function $F: M^{+}(T) \rightarrow M^{+}(X)$ defined by $F(\mu)=\mu \circ \phi_{\mu}^{-1}$ is linear and strongly continuous.

Proof. If $\mu \in M^{+}(T)$ and $a \geq 0$, then $a \mu \ll \mu$, implying $\phi_{a \mu}=\phi_{\mu} a \mu$-a.e. Thus

$$
F(a \mu)=a \mu \circ \phi_{a \mu}^{-1}=a \mu \circ \phi_{\mu}^{-1}=a F(\mu) .
$$

Likewise, if $\nu, \mu \in M^{+}(T)$, then both $\nu$ and $\mu$ are absolutely continuous with respect to $\mu+\nu$. Thus, $\phi_{\mu+\nu}=\phi_{\mu} \mu$-a.e. and $\phi_{\mu+\nu}=\phi_{\nu} \nu$-a.e., implying

$$
\begin{aligned}
F(\mu+\nu) & =(\mu+\nu) \circ \phi_{\mu+\nu}^{-1}=\mu \circ \phi_{\mu+\nu}^{-1}+\nu \circ \phi_{\mu+\nu}^{-1} \\
& =\mu \circ \phi_{\mu}^{-1}+\nu \circ \phi_{\nu}^{-1}=F(\mu)+F(\nu) .
\end{aligned}
$$

Thus $F$ is linear. Also, $\|F(\mu)-F(\nu)\|=\left\|(\mu-\nu) \circ \phi_{\mu+\nu}^{-1}\right\| \leq\|\mu-\nu\|$, thus $F$ is strongly Lipschitz.

The following two lemmas and Theorems 6.5 and 6.6 explore some closure properties of the set of all possible multispectrums.

Lemma 6.3. If $\mu, \nu \in M^{+}(T)$ and $\nu \ll \mu$ with $\frac{d \nu}{d \mu}=f$, then $\nu_{\omega_{1}} \ll \mu_{\omega_{1}}$ and for $\mu_{\omega_{1}}$-a.e. $\langle t, \mathbf{s}\rangle \in T \times[0, \infty]^{\omega_{1}}$,

$$
\frac{d \nu_{\omega_{1}}}{d \mu_{\omega_{1}}}(t, \mathbf{s})=f(t)
$$


Proof. Since $\nu \ll \mu, \phi_{\omega_{1}, \nu}=\phi_{\omega_{1}, \mu} \nu$-a.e. (See Theorem 4.3, (4.7).) For bounded Borel $H: T \times[0, \infty]^{\omega_{1}} \rightarrow \mathbb{R}$ with $\phi_{\omega_{1}}=\phi_{\omega_{1}, \mu}$, we have

$$
\int H d \nu_{\omega_{1}}=\int H\left(t, \phi_{\omega_{1}}(t)\right) d \nu(t)=\int H\left(t, \phi_{\omega_{1}}(t)\right) f(t) d \mu(t)=\int H(t, \mathbf{s}) f(t) d \mu_{\omega_{1}}(t, \mathbf{s}) \text {. }
$$

Lemma 6.4. If $\mu \in M^{+}(T)$ and $G$ is a non-negative $\sigma_{\omega_{1}}(\mu)$-integrable function, then the measure $\nu \in M^{+}(T)$ defined by the weak* integral formula

$$
\nu=w^{*}-\int \mu_{\omega_{1}, \mathbf{s}} G(\mathbf{s}) d \sigma_{\omega_{1}}(\mu)(\mathbf{s}),
$$

is absolutely continuous with respect to $\mu$ and $\frac{d \nu}{d \mu}=G \circ \phi_{\mu, \omega_{1}}$.

Proof. Using the definition of $\nu$ and (4.6) of Theorem 4.3, for any $H \in C(T)$,

$$
\int H d \nu=\iint H(t) G(\mathbf{s}) d \mu_{\omega_{1}, \mathbf{s}}(t) d \sigma_{\omega_{1}}(\mu)(\mathbf{s})=\int H(t) G \circ \phi_{\mu, \omega_{1}}(t) d \mu(t) .
$$

Definition 6.2. Let $\mathcal{S}_{(T, \rho)}$ denote the set of all $\omega_{1}$-order multispectrums of $T$, $\mathcal{S}_{(T, \rho)}=\left\{\sigma_{\omega_{1}}(\mu): \mu \in M^{+}(T)\right\}$

Theorem 6.5. The set $\mathcal{S}_{(T, \rho)}$ is a strongly closed subcone of $M^{+}\left([0, \infty]^{\omega_{1}}\right)$. Further, if $\kappa \in \mathcal{S}_{(T, \rho)}, \xi \in M^{+}\left([0, \infty]^{\omega_{1}}\right)$, and $\xi \ll \kappa$, then $\xi \in \mathcal{S}_{(T, \rho)}$.

Proof. Since $\sigma(\cdot)_{\omega_{1}}$ is a positive linear functional on $M^{+}(T), \mathcal{S}_{(T, \rho)}=\sigma_{\omega_{1}}\left(M^{+}(T)\right)$ is a subcone of $M^{+}\left([0, \infty]^{\omega_{1}}\right)$.

Suppose $\kappa \in \mathcal{S}_{(T, \rho)}, \xi \in M^{+}\left([0, \infty]^{\omega_{1}}\right)$, and $\xi \ll \kappa$. Let $G=\frac{d \xi}{d \kappa}$ and $\mu \in M^{+}(T)$ so that $\kappa=\sigma_{\omega_{1}}(\mu)$ and let $\phi_{\mu, \omega_{1}}$ be as in Theorem 4.3. By Lemma 6.4, the measure $\nu$ defined by the weak* integral $w^{*}-\int \mu_{\omega_{1}, \mathbf{s}} G(\mathbf{s}) d \kappa(s)$ is absolutely continuous with respect to $\mu$ and $\frac{d \nu}{d \mu}=G \circ \phi_{\mu, \omega_{1}}$. Thus by Lemma $6.3, \nu_{\omega_{1}} \ll \mu_{\omega_{1}}$ and

$$
\frac{d \nu_{\omega_{1}}}{d \mu_{\omega_{1}}}(t, \mathbf{s})=G \circ \phi_{\mu, \omega_{1}}(t) .
$$

For $H \in C\left([0, \infty]^{\omega_{1}}\right)$,

$$
\begin{aligned}
\int H d \sigma_{\omega_{1}}(\nu) & =\int H \circ \zeta_{\omega_{1}} d \nu_{\omega_{1}}=\int H(\mathbf{s}) G \circ \phi_{\mu, \omega_{1}}(t) d \mu_{\omega_{1}}(t, \mathbf{s}) \\
& =\int H \circ \phi_{\mu, \omega_{1}}(t) G \circ \phi_{\mu, \omega_{1}}(t) d \mu(t) \\
& =\int H G d \sigma_{\omega_{1}}(\mu)=\int H G d \kappa=\int H d \xi
\end{aligned}
$$

where the first equality is the definition of $\sigma_{\omega_{1}}(\nu)$, the second is $(6.1)$, the third is (4.6) of Theorem 4.3 as is the fourth, the fifth from the choice of $\mu$, and the last by the definition of $G$. Thus $\xi=\sigma_{\omega_{1}}(\nu)$, implying $\xi \in \mathcal{S}_{(T, \rho)}$.

Now it will be shown that $\mathcal{S}_{(T, \rho)}$ is strongly closed. Suppose $\left\langle\kappa_{i}\right\rangle_{i=1}^{\infty}$ is a sequence of elements of $\mathcal{S}_{(T, \rho)}$ which converge strongly to the finite regular Borel measure $\kappa$. For each $i$, let $\mu_{i}$ denote an $M^{+}(T)$ so that $\kappa_{i}=\sigma_{\omega_{1}}\left(\mu_{i}\right)$. Now $\sup _{i} \mu_{i}(T)=$ $\sup _{i} \kappa_{i}\left([0, \infty]^{\omega_{1}}\right)<\infty$, so the sum $\sum_{i=1}^{\infty} \frac{1}{2^{i}} \mu_{i}$ converges strongly to an element $\mu$ of $M^{+}(T)$. Since $(\cdot)_{\omega_{1}}$ is strongly continuous,

$$
\sigma_{\omega_{1}}(\mu)=\sum_{i=1}^{\infty} \frac{1}{2^{i}} \sigma_{\omega_{1}}\left(\mu_{i}\right)=\sum_{i=1}^{\infty} \frac{1}{2^{i}} \kappa_{i} .
$$

Thus, $\sum_{i=1}^{\infty} \frac{1}{2^{i}} \kappa_{i} \in \mathcal{S}_{(T, \rho)}$. Since $\kappa \ll \sum_{i=1}^{\infty} \frac{1}{2^{i}} \kappa_{i}, \kappa \in \mathcal{S}_{(T, \rho)}$. 
Theorem 6.6. If $\kappa$ is a finite regular Borel measure on $[0, \infty]^{\omega_{1}}$ so that for every Borel $A$ with $\kappa(A)>0$ there exists a $\kappa_{A} \in \mathcal{S}_{(T, \rho)}$ so that $\kappa_{A} \ll \kappa$ and $\kappa_{A}(A)>0$, then $\kappa \in \mathcal{S}_{(T, \rho)}$.

Proof. For any Borel $A \subseteq[0, \infty]^{\omega_{1}}$, let $\kappa 1_{A}$ denote the measure which is absolutely continuous with respect to $\kappa$ and has Radon-Nikodym derivative $1_{A}$. Let $\mathcal{C}=\left\{A \subseteq[0, \infty]^{\omega_{1}}: A\right.$ is Borel and $\left.\kappa 1_{A} \in \mathcal{S}_{(T, \rho)}\right\}$. Easily if $\left\{A_{i}\right\}_{i=1}^{\infty}$ is a countable collection of elements of $\mathcal{C}$, then $\sum_{i=1}^{\infty} \frac{1}{2^{2}} \kappa 1_{A_{i}}$ converges strongly to a finite measure in $M\left([0, \infty]^{\omega_{1}}\right)$. Since $\mathcal{S}_{(T, \rho)}$ is a strongly closed subcone of $M\left([0, \infty]^{\omega_{1}}\right)$, and each term of $\sum_{i=1}^{\infty} \frac{1}{2^{i}} \kappa 1_{A_{i}}$ is in $\mathcal{S}_{(T, \rho)}, \sum_{i=1}^{\infty} \frac{1}{2^{i}} \kappa 1_{A_{i}}$ must also be in $\mathcal{S}_{(T, \rho)}$. Since $\kappa 1_{\bigcup_{i=1}^{\infty} A_{i}} \ll \sum_{i=1}^{\infty} \frac{1}{2^{i}} \kappa 1_{A_{i}}$, Theorem 6.5 implies $\kappa 1_{\cup_{i=1}^{\infty} A_{i}} \in \mathcal{S}_{(T, \rho)}$ and $\bigcup_{i=1}^{\infty} A_{i} \in \mathcal{C}$. That is, $\mathcal{C}$ is closed under countable unions. It follows that there is an element $B$ of $\mathcal{C}$ so that

$$
\kappa(B)=\sup _{A \in \mathcal{C}} \kappa(A)
$$

It is enough to show $\kappa(B)=\kappa\left([0, \infty]^{\omega_{1}}\right)$ to arrive at the conclusion of the theorem.

Suppose $\kappa(B)<\kappa\left([0, \infty]^{\omega_{1}}\right)$. Then $\kappa\left([0, \infty]^{\omega_{1}} \backslash B\right)>0$ implying there is a

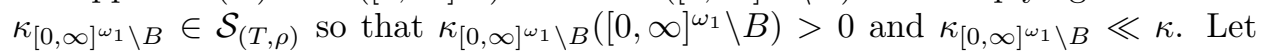
$G$ be the Radon-Nikodym derivative of $\kappa_{[0, \infty]^{\omega_{1}} \backslash B}$ with respect to $\kappa$, and let $C=$ $\left\{\mathbf{s} \in[0, \infty]^{\omega_{1}} \backslash B: G(\mathbf{s})>0\right\}$. Easily $\kappa 1_{C} \ll \kappa_{[0, \infty]^{\omega_{1} \backslash B}}$, and so $\kappa 1_{C} \in \mathcal{S}_{(T, \rho)}$. Thus, $B \cup C \in \mathcal{C}$. But $\kappa_{[0, \infty]^{\omega_{1}} \backslash B}(C)=\kappa_{[0, \infty]^{\omega_{1} \backslash B}\left([0, \infty]^{\omega_{1}} \backslash B\right)>0 \text {, implying }}$ $\kappa(B \cup C)>\kappa(B)$, contradicting (6.2).

The following lemma uses the homogeneity of the operator $K$ to make some small calculations. The corollary which follows can be thought of as a characterization of the uniqueness of $\mu_{\alpha}$, which is a transfinite version of Theorem 2.2.

Theorem 6.7. Let $\alpha \geq 1$. Suppose $\nu \in M^{+}\left([0, \infty]^{\alpha}\right)$, for each $\mathbf{s} \in[0, \infty]^{\alpha}, \lambda_{\mathbf{s}}$ is in $M^{+}(T)$ and the map ' $\mathbf{s} \rightarrow \lambda_{\mathbf{s}}$ ' is weak $k^{*}$ Borel and $\int \lambda_{\mathbf{s}}(T) d \nu(\mathbf{s})<\infty$. Further let $\mu=w^{*}-\int \lambda_{\mathbf{s}} d \nu(\mathbf{s})$ and suppose the following two conditions hold:

(1) $K(\mu)=w^{*}-\int \lambda_{\mathbf{s}} \times \delta_{s_{0}} d \nu(\mathbf{s})$ and

(2) for $1 \leq \beta<\alpha$, for $\nu \circ \theta_{\alpha, \beta}^{-1}$-a.e.-u,

$$
K\left(w^{*}-\int \lambda_{\mathbf{s}} d \nu_{\theta_{a, \beta=\mathbf{u}}}(\mathbf{s})\right)=w^{*}-\int \lambda_{\mathbf{s}} \times \delta_{s_{\beta}} d \nu_{\theta_{\alpha, \beta}=\mathbf{u}}(\mathbf{s}) .
$$

Then, for $1 \leq \beta \leq \alpha, \mu_{\beta}=w^{*}-\int \lambda_{\mathbf{s}} \times \delta_{\mathbf{s} \mid \beta} d \nu(\mathbf{s})$.

Proof. That the conclusion is true for $\beta=1$ is just condition (1).

Induction Hypothesis: Suppose that the conclusion is true for all $\gamma<\beta$.

Case: $\beta$ is a limit ordinal.

Let $\xi=w^{*}-\int \lambda_{\mathbf{s}} \times \delta_{\mathbf{s} \mid \beta} d \nu(\mathbf{s})$. Easily, $\xi \circ \theta_{\beta, \gamma}^{-1}=w^{*}-\int \lambda_{\mathbf{s}} \times \delta_{\mathbf{s} \mid \gamma} d \nu(\mathbf{s})$, which equals $\xi_{\gamma}$ by the induction hypothesis. So $\xi=\mu_{\beta}$ by uniqueness of Kolmogoroff extensions.

Case: $\beta=\gamma+1$.

By the induction hypothesis, $\mu_{\gamma}=w^{*}-\int \lambda_{\mathbf{s}} \times \delta_{\mathbf{s} \mid \gamma} d \nu(\mathbf{s})$. So

$$
\mu_{\gamma} \circ \zeta_{\gamma}^{-1}=w^{*}-\int\left[\int \lambda_{\mathbf{s}}(T) d \nu_{\theta_{\alpha, \beta}=\mathbf{u}}(\mathbf{s})\right] \delta_{\mathbf{u}} d \nu \circ \theta_{\alpha, \gamma}^{-1}(\mathbf{u})
$$

and $\mu_{\gamma} \circ \zeta_{\gamma}^{-1}$-a.e.-u,

$$
\mu_{\gamma, \mathbf{u}}=\frac{w^{*}-\int \lambda_{\mathbf{s}} d \nu_{\theta_{\alpha, \gamma}=\mathbf{u}}(\mathbf{s})}{\int \lambda_{\mathbf{s}}(T) d \nu_{\theta_{\alpha, \gamma}=\mathbf{u}}(\mathbf{s})}
$$


Using the homogeneity of the operator $K,(6.4)$, and hypothesis (2), we have $\mu_{\gamma} \circ$ $\zeta_{\gamma}^{-1}$-a.e.-u,

$$
K\left(\mu_{\gamma, \mathbf{u}}\right)=\frac{w^{*}-\int \lambda_{\mathbf{s}} \times \delta_{s_{\gamma}} d \nu_{\theta_{\alpha, \gamma}=\mathbf{u}}(\mathbf{s})}{\int \lambda_{\mathbf{s}}(T) d \nu_{\theta_{\alpha, \gamma}=\mathbf{u}}(\mathbf{s})} .
$$

Using (6.3) and (6.5), the case follows.

Corollary 6.8. For $1 \leq \alpha<\omega_{1}$, the measure $\mu_{\alpha}$ is the only measure $\lambda$ on $T \times$ $[0, \infty]^{\alpha}$ which satisfies the following: For $\left\langle\lambda_{\mathbf{s}}\right\rangle_{\mathbf{s} \in[0, \infty]^{\alpha}}$ the factor kernel of $\lambda$ with respect to $\zeta_{\alpha}$, and $\nu=\lambda \circ \zeta_{\alpha}^{-1}$ the following two conditions hold:

(1) $K(\mu)=w^{*}-\int \lambda_{\mathbf{s}} \times \delta_{s_{0}} d \nu(\mathbf{s})$,

(2) for $1 \leq \beta<\alpha$, for $\nu \circ \theta_{\alpha, \beta}^{-1}$-a.e.-u,

$$
K\left(w^{*}-\int \lambda_{\mathbf{s}} d \nu_{\theta_{a, \beta=\mathbf{u}}}(\mathbf{s})\right)=w^{*}-\int \lambda_{\mathbf{s}} \times \delta_{s_{\beta}} d \nu_{\theta_{\alpha, \beta}=\mathbf{u}}(\mathbf{s}) .
$$

Proof. If $\lambda$ satisfies (1) and (2), then Theorem 6.7 implies $\lambda=\mu_{\alpha}$. All that is left to show is (1) and (2) is true for $\mu_{\alpha}$. It follows from Theorem 3.1 statement (3.3) and an obvious manipulation of coordinates, that for $1 \leq \beta<\alpha$,

$$
\mu_{\beta}=\mu_{\alpha} \circ \pi_{\alpha, \beta}^{-1}=w^{*}-\int \mu_{\alpha, \mathbf{s}} \times \delta_{\theta_{\alpha, \beta}(\mathbf{s})} d \mu_{\alpha} \circ \zeta_{\alpha}^{-1}(\mathbf{s}) .
$$

where "s $\rightarrow \mu_{\alpha, \mathbf{s}}$ " is the factor kernel of $\mu_{\alpha}$ with respect to $\zeta_{\alpha}$. Since $K(\mu)=\mu_{1}$, (6.6) (with $\beta=1$ ) implies (1). All that is left is to show (2) is true for $\mu_{\alpha}$. Remember by definition $\sigma_{\alpha}(\mu)=\mu_{\alpha} \circ \zeta_{\alpha}^{-1}$ and (3.7) states $\sigma_{\beta}(\mu)=\sigma_{\alpha}(\mu) \circ \theta_{\alpha, \beta}^{-1}$ for $\beta<\alpha$. First note that (6.6) implies $\sigma_{\beta}(\mu)$-a.e.-u,

$$
\mu_{\beta, \mathbf{u}}=w^{*}-\int \mu_{\alpha, \mathbf{s}} d \sigma_{\alpha}(\mu)_{\theta_{\alpha, \beta}=\mathbf{u}}(\mathbf{s}) .
$$

By definition of $\mu_{\beta+1}$ and (6.7), for $h \in C\left(T \times[0, \infty]^{\beta+1}\right)$,

$$
\begin{aligned}
\int h d \mu_{\beta+1} & =\iint h(t, \mathbf{u} * x) d K\left(\mu_{\beta, \mathbf{u}}\right)(t, x) d \sigma_{\beta}(\mu)(\mathbf{u}) \\
& =\iint h(t, \mathbf{u} * x) d K\left(\int \mu_{\alpha, \mathbf{s}} d \sigma_{\alpha}(\mu)_{\theta_{\alpha, \beta}=\mathbf{u}}(\mathbf{s})\right)(t, x) d \sigma_{\beta}(\mu)(\mathbf{u}) .
\end{aligned}
$$

But it follows from (6.6) that

$$
\begin{aligned}
\int h d \mu_{\beta+1} & =\iint h\left(t, \theta_{\alpha, \beta+1}(\mathbf{s})\right) d \mu_{\alpha, \mathbf{s}}(t) d \sigma_{\alpha}(\mu)(\mathbf{s}) \\
& =\iiint h(t, \mathbf{u} * x) d \mu_{\alpha, \mathbf{s}}(t) d \sigma_{\alpha}(\mu)_{\theta_{\alpha, \beta}=\mathbf{u}}(\mathbf{s}) d \sigma_{\beta}(\mu)(\mathbf{u}) .
\end{aligned}
$$

The equations (6.8) and (6.9) together imply (2).

\section{Multiplicative Cascades in Certain Ultrametric Spaces}

In this section, a set valued cascade and an associated multiplicative process are analyzed in order to show the possibility of producing, in certain ultrametric spaces, the 'bits and pieces' of $\alpha$-order basic multispectrums needed in Theorem 6.6. In particular we have Theorem 7.7 at the end of the section as the main theorem. Let $b$ be an integer $\geq 2, T$ be the product space $\{0,1, \ldots, b-1\}^{\mathbb{N}}, d>0$, and $\rho_{d}: T \times T \rightarrow[0, \infty)$ be the metric defined by $\rho_{d}\left(\left\langle t_{i}\right\rangle_{i=1}^{\infty},\left\langle s_{i}\right\rangle_{i=1}^{\infty}\right)=b^{-d k}$ where $k=\inf \left\{j: s_{j} \neq t_{j}\right\}$. The function $\rho_{d}$ is an ultrametric compatible with the product 
topology on $\dot{T}$. Let $\psi_{d}$ be the dimension function relative to $\rho_{d}$ and $\Phi_{d}$ be the local dimension function relative to $\rho_{d}$. Then $\psi_{d}(T)=d$. For $a, b \in(0, \infty)$,

$$
\psi_{a}=\frac{a}{b} \psi_{b} \text { and } \Phi_{a}=\frac{a}{b} \Phi_{b} .
$$

Let $T^{*}$ denote the set of all finite sequences of elements in $\{0,1, \ldots, b-1\}$ with $\emptyset$ denoting the empty sequence. For any $\tau \in T^{*} \cup T$, let $|\tau|$ denote the length of $\tau$. For either $t \in T$ or $t \in T^{*}$, and $n$ an integer less than or equal to the length of $t$, then $\left.t\right|_{n}$ will denote the element in $T^{*}$ of length $n$ which has coordinates equal to the first $n$ coordinates of $t$.

Also we let $\lambda$ denote normalized Haar measure on $T$, where $T$ is viewed as infinite product group of the finite group $\mathbb{Z} \bmod b$.

Definition 7.1. Let $\kappa$ be a basic multispectrum of diffuseness order $\alpha \geq 1$, with spreading measure $\nu$ and unidimensional map $U$. Let $\left\langle\mathcal{P}_{n}\right\rangle_{n=0}^{\infty}$ be a sequence of partitions of $[0, d]^{\alpha}$ into $\nu$-measurable sets so that

(1) $\mathcal{P}_{0}=\left\{[0, d]^{\alpha}\right\}$,

(2) the $\sigma$-algebra generated by the set $\bigcup_{n=0}^{\infty} \mathcal{P}_{n}$ and the $\nu$-null sets is the $\nu$ measurable sets,

(3) for each $n, \mathcal{P}_{n+1}$ refines $\mathcal{P}_{n}$, and

(4) if $A \in \bigcup_{n=0}^{\infty} \mathcal{P}_{n}$, then $\nu(A)>0$.

Such a sequence will be called a positive filtration base for $\nu$ (or for $\kappa$ ). For any $\mathbf{s} \in[0, d]^{\alpha}$ and $n \in \mathbb{Z}^{+}$, let $\mathcal{E}_{n}(\mathbf{s})$ denote the unique element in $\mathcal{P}_{n}$ satisfying $\mathbf{s} \in \mathcal{E}_{n}(\mathbf{s})$. A positive filtration base $\left\langle\mathcal{P}_{n}\right\rangle_{n=0}^{\infty}$ is said to encode a coordinate induction rule for $\kappa$ if and only if $\nu$-a.e.-s, for each $\beta<\alpha$,

$$
\begin{aligned}
s_{0}-d \cdot \limsup _{n \rightarrow \infty} \frac{1}{n} \log _{b}\left(\frac{\nu\left(\mathcal{E}_{n}(\mathbf{s})\left|\theta_{\alpha, \beta}=\mathbf{s}\right|_{\beta}\right)}{\nu\left(\mathcal{E}_{n}(\mathbf{s})\right)}\right) & =s_{\beta} \\
\text { and } s_{0}-d \cdot \limsup _{n \rightarrow \infty} \frac{1}{n} \log _{b}\left(\frac{1}{\nu\left(\mathcal{E}_{n}(\mathbf{s})\right)}\right) & =U(\mathbf{s}),
\end{aligned}
$$

where $s_{\beta}$ is the coordinate of $\mathbf{s}$ of index $\beta$ and $\left.\mathbf{s}\right|_{\beta}=\theta_{\alpha, \beta}(\mathbf{s})$. A function $r$ from $\{\emptyset\} \cup\left\langle\mathcal{P}_{n}\right\rangle_{n=0}^{\infty}$ into $\{0\} \cup[1, \infty)$ which is $1-1$ and $r(\emptyset)=0$ is said to be a transfer map for $\left\langle\mathcal{P}_{n}\right\rangle_{n=0}^{\infty}$. A transfer map $r$ is said to encode an induction initiation rule if for $\nu$-a.e.-s,

$$
d-d \cdot \lim _{n \rightarrow \infty} \log _{b}\left(r\left(\mathcal{E}_{n}(\mathbf{s})\right)\right)=s_{0} .
$$

A pair $\left\langle\left\langle\mathcal{P}_{n}\right\rangle_{n=0}^{\infty}, r\right\rangle$ is called a coordinate induction pair for $\kappa$ if $\left\langle\mathcal{P}_{n}\right\rangle_{n=0}^{\infty}$ is a positive filtration base which encodes a coordinate induction rule and $r$ is a transfer map for $\left\langle\mathcal{P}_{n}\right\rangle_{n=0}^{\infty}$ which encodes an induction initiation.

Definition 7.2. Given a positive filtration base $\left\langle\mathcal{P}_{n}\right\rangle_{n=0}^{\infty}$ for some basic multispectrum $\kappa$ of spreading order $\geq 1$, and a transfer map $r$, for each $n \geq 1$, the $\mathbf{n}^{\text {th }}$ transition matrix induced by the pair $\left\langle\left\langle\mathcal{P}_{n}\right\rangle_{n=0}^{\infty}, r\right\rangle$ is the matrix $q_{n}=\left(q_{n, A, B}\right)_{A, B}$ with rows indexed by elements of $\mathcal{P}_{n-1} \cup\{\emptyset\}$ and columns indexed by elements of $\mathcal{P}_{n} \cup\{\emptyset\}$ satisfying: for $A \in \mathcal{P}_{n-1} \cup\{\emptyset\}$ and $B \in \mathcal{P}_{n} \cup\{\emptyset\}$,

$$
q_{n, A, B}=\left\{\begin{array}{cl}
r(B)^{-1} \nu(B \mid A) & \text { if } B \neq \emptyset \neq A, \\
1-\sum_{B \in \mathcal{P}_{n}} r(B)^{-1} \nu(B \mid A) & \text { if } B=\emptyset \neq A, \\
1 & \text { if } B=\emptyset=A, \\
0 & \text { if } B \neq \emptyset=A,
\end{array}\right.
$$


and an initial distribution induced by $r, q_{0}$, is defined on $\mathcal{P}_{0} \cup\{\emptyset\}$ by

$$
\begin{aligned}
q_{0,[0, d]^{\alpha}} & =r\left([0, d]^{\alpha}\right)^{-1}, \\
q_{0, \emptyset} & =1-r\left([0, d]^{\alpha}\right)^{-1} .
\end{aligned}
$$

Lemma 7.1. The vector $q_{0}$ defined in (7.4) is a probability vector on $\{\emptyset\} \cup \mathcal{P}_{0}$ and for all $n \in \mathbb{N}$, the matrix $q_{n}$ defined in (7.3) is a probability transition matrix from $\{\emptyset\} \cup \mathcal{P}_{n-1}$ to $\{\emptyset\} \cup \mathcal{P}_{n}$. Further, for $A$ a Borel subset of $[0, d]$ and $B \in \mathcal{P}_{n-1}$,

$$
\nu(A \mid B)=\sum_{C \in \mathcal{P}_{n}} r(C) \nu(A \mid C) q_{n, B, C} .
$$

Proof. Since $r(A) \geq 1$ for $A \neq \emptyset$, the numbers $q_{n, A, B}$ and $q_{0, A}$ are in $[0,1]$. A direct check using the definitions will show $\sum_{B} q_{n, A, B}=1$ and $\sum_{A} q_{0, A}=1$. Thus the first statement holds. For the second statement note that

$$
\sum_{C \in \mathcal{P}_{n}} r(C) \nu(A \mid C) q_{n, B, C}=\sum_{C \in \mathcal{P}_{n}} \nu(A \mid C) \nu(C \mid B) .
$$

Since for all $C \in \mathcal{P}_{n}$, either $C \subseteq B$ or $C \cap B=\emptyset$, the set $\left\{C \in \mathcal{P}_{n}: C \subseteq B\right\}$ is a countable partition of $B$, and $\nu(C \mid B)=0$ for $C \in\left\{C \in \mathcal{P}_{n}: C \cap B \neq \emptyset\right\}$. Thus the right hand side of $(7.5)$ equals $\nu(A \mid B)$.

The sequence of matrices $\left\langle q_{n}\right\rangle_{n=0}^{\infty}$ allows us to define a distribution for a set valued stochastic process $\left\langle A_{n}\right\rangle$ where the value of $A_{n}$ is in $\{\emptyset\} \cup \mathcal{P}_{n}$ by the consistency scheme $P\left(A_{0}=B\right)=q_{0, B}$ and the $n^{t h}$ transition defined by

$$
P\left(A_{n}=B_{n} \mid A_{i}=B_{i}, i<n\right)=q_{n, B_{n-1}, B_{n}} .
$$

Also this defines a distribution for a real valued stochastic process as the distribution of the transferred sequence $\left\langle r\left(A_{n}\right)\right\rangle_{n=0}^{\infty}$. We can use the transferred distribution on $\left\langle r\left(A_{n}\right)\right\rangle_{n=0}^{\infty}$ as a generator process for a random cascade (see the paragraph after Theorem 2.1 of [21], and also Definition 2.1 and Theorem 2.1 of the same.) The following two definitions give the notation of section 2 of [21] in our coordinate induction pair setting.

Definition 7.3. Given a positive filtration base $\left\langle\mathcal{P}_{n}\right\rangle_{n=0}^{\infty}$ for a basic multispectrum $\kappa$, let $\Omega_{\left\langle\mathcal{P}_{n}\right\rangle_{n=0}^{\infty}}$ denote the product space $\prod_{\tau \in T^{*}} X_{\tau}$, where for each $\tau \in T^{*}$, $X_{\tau}=\{\emptyset\} \cup \mathcal{P}_{|\tau|}$. Endowing each $\{\emptyset\} \cup \mathcal{P}_{|\tau|}$ with the discrete topology and $\Omega_{\left\langle\mathcal{P}_{n}\right\rangle_{n=0}^{\infty}}$ with the induced product topology, $\Omega_{\left\langle\mathcal{P}_{n}\right\rangle_{n=0}^{\infty}}$ is a separable completely metrizable space. Let $\mathcal{F}$ be the Borel field of this $\bar{\Omega}_{\left\langle\mathcal{P}_{n}\right\rangle_{n=0}^{\infty}}$. For each $\tau \in T^{*}$, let $A_{\tau}$ : $\Omega_{\left\langle\mathcal{P}_{n}\right\rangle_{n=0}^{\infty}} \rightarrow\{\emptyset\} \cup \mathcal{P}_{|\tau|}$ be the $\tau^{\text {th }}$ coordinate projection. The collection $\left\langle A_{\tau}\right\rangle_{\tau \in T^{*}}$ is called the set valued random cascade associated with $\left\langle\mathcal{P}_{n}\right\rangle_{n=0}^{\infty}$. Given a transfer map $r$ for $\left\langle\mathcal{P}_{n}\right\rangle_{n=0}^{\infty}$, the probability cascade measure generated by the pair $\left\langle\left\langle\mathcal{P}_{n}\right\rangle_{n=0}^{\infty}, r\right\rangle$ is the measure on the Borel sets of $\Omega_{\left\langle\mathcal{P}_{n}\right\rangle_{n=0}^{\infty}}$ defined by the following scheme:

(1) $P\left(A_{\emptyset}\right)=q_{0, B}$ if $B \in\left\{\emptyset,[0, \infty]^{\alpha}\right\}$, and

(2) If $B_{\tau} \in\{\emptyset\} \cup \mathcal{P}_{|\tau|}$ for all $\tau$ satisfying $|\tau| \leq n$, then

$$
P\left(A_{\tau}=B_{\tau} \text { for }|\tau|=n \mid A_{\eta}=B_{\eta} \text { for }|\eta|<n\right)=\prod_{\tau:|\tau|=n} q_{n, A_{\tau \mid n-1}, A_{\tau}} .
$$

Theorem 7.2. Let $\left\langle\left\langle\mathcal{P}_{n}\right\rangle_{n=0}^{\infty}, r\right\rangle$ and $\left\langle A_{\tau}\right\rangle_{\tau \in T^{*}}$ be as in Definition 7.3. For each $\tau \in T^{*}$, define a non-negative random variable $W_{\tau}=r \circ A_{\tau}$. The collection 
$\left\langle W_{\tau}\right\rangle_{\tau \in T^{*}}$ is a random cascade with a tree distribution with cascade generator $\left\langle q_{n}\right\rangle$ in the sense of Definition 2.1 of [21]. The function valued process, $\left\langle Q_{n}: \Omega_{\left\langle\mathcal{P}_{n}\right\rangle_{n=0}^{\infty}} \times T \rightarrow[0, \infty)\right\rangle_{n=0}^{\infty}$, defined by

$$
Q_{n}(\omega, t)=\prod_{i=0}^{n} W_{t \mid i}(\omega)
$$

is a multiplicative cascade in the sense of statements 2.2 through 2.4 of [21].

Proof. Transferring back and forth from the set values $E$ and the real numbers $r(E)$ and letting $q_{n}\left(r\left(B_{n}\right) \mid r\left(B_{0}\right), r\left(B_{1}\right), \cdots, r\left(B_{n-1}\right)\right)=q_{n, B_{n-1, B_{n}}}$, (7.3) implies

$$
1_{B \neq \emptyset}=\sum_{C \in \mathcal{P}_{n+1}} r(C) q_{n, B, C}
$$

which is equation 2.1c of [21]. Also equation (i) of [21] follows from the equation: $1=r\left([0, d]^{\alpha}\right) q_{0,[0, d]^{\alpha}}$. Establishing that the transferred distribution satisfies (ii) follows from (7.6).

We will use the following defined setup throughout this section:

Definition 7.4. Let $\kappa$ be a basic multispectrum of spreading order $\alpha \geq 1$. A $\kappa$-cascade-setup is the ordered collection of objects $\left\langle\kappa,\left\langle\mathcal{P}_{n}\right\rangle_{n=0}^{\infty}, r,\left\langle A_{\tau}\right\rangle_{\tau \in T^{*}}\right.$, $\left\langle W_{\tau}\right\rangle_{\tau \in T^{*}},\left\langle\Omega_{\left\langle\mathcal{P}_{n}\right\rangle_{n=0}^{\infty}}, \mathcal{F}, P\right\rangle,\left\langle\mathcal{F}_{n}\right\rangle_{n=0}^{\infty},\left\langle Q_{n}\right\rangle_{n=0}^{\infty}>$ where $\left\langle\mathcal{P}_{n}\right\rangle_{n=0}^{\infty}$ is a positive filtration base for $\kappa, r$ is a transfer map for $\left\langle\mathcal{P}_{n}\right\rangle_{n=0}^{\infty},\left\langle\Omega_{\left\langle\mathcal{P}_{n}\right\rangle_{n=0}^{\infty}}, \mathcal{F}, P\right\rangle$ is the probability space generated by the pair $\left\langle\left\langle\mathcal{P}_{n}\right\rangle_{n=0}^{\infty}, r\right\rangle,\left\langle A_{\tau}\right\rangle_{\tau \in T^{*}}$ and $\left\langle W_{\tau}\right\rangle_{\tau \in T^{*}}$ are the set-valued cascade and random cascade associated with $\left\langle\left\langle\mathcal{P}_{n}\right\rangle_{n=0}^{\infty}, r\right\rangle,\left\langle\mathcal{F}_{n}\right\rangle_{n=0}^{\infty}$ is the filtration defined by $\mathcal{F}_{n}=\sigma-\left\{W_{\tau}:|\tau| \leq n\right\}$, and $\left\langle Q_{n}\right\rangle_{n=0}^{\infty}$ is the multiplicative process generated by the pair $\left\langle\left\langle\mathcal{P}_{n}\right\rangle_{n=0}^{\infty}, r\right\rangle$.

Since $r$ is $1-1, \mathcal{F}_{n}=\sigma-\left\{A_{\tau}:|\tau| \leq n\right\}$ also.

One of the most exploited properties of the multiplicative process $\left\langle Q_{n}\right\rangle_{n=0}^{\infty}$ defined above is its being a positive $T$-martingale: That is, $\left\langle Q_{n}\right\rangle_{n=0}^{\infty}$ is a sequence of non-negative functions measurable with respect to the $\sigma$-algebra generated by the $\mathcal{F} \times \mathcal{B}(T)$-rectangles ${ }^{5}$ and for each $t \in T,\left\langle Q_{n}(t)\right\rangle_{n=0}^{\infty}$ is a martingale with respect to the measure $P$. (See [9] and [18] for basic facts concerning positive $T$-martingales and sections 1, 2, and 3 of [21] for some theory for positive $T$-martingales with this cascade structure.) Perturbations of multiplicative cascades $Q_{n}$ which preserve the 'cascade measurability' and T-martingale properties can be obtained by weight systems, $\mathbb{G}=\left\langle G_{\tau}\right\rangle_{\tau \in T^{*}}$, as introduced in Definition 2.2 of [21].

Lemma 7.3. Fix a $\kappa$-cascade-setup. Let $B$ be a Borel subset of $[0, d]^{\alpha}$, and let $\mathbb{F}(B)=\left\langle F(B)_{\tau}\right\rangle_{\tau \in T^{*}}$, where

$$
F(B)_{\tau}=\left\{\begin{array}{cl}
\nu\left(B \mid A_{\tau}\right) & \text { if } A_{\tau} \neq \emptyset \\
0 & \text { if } A_{\tau}=\emptyset
\end{array}\right.
$$

Then $\mathbb{F}(B)$ is a weight system for the multiplicative process $\left\langle Q_{n}\right\rangle_{n=0}^{\infty}$.

\footnotetext{
${ }^{5}$ Dan Mauldin has pointed out that joint measurablity is needed here. This was not noted in earlier work.
} 
Proof. Fix $t \in T$, and $n \in \mathbb{N}$, then

$$
\begin{aligned}
E_{P}\left[Q_{n+1}(t) F(B)_{t \mid n+1} \mid \mathcal{F}_{n}\right] & =Q_{n}(t) \cdot E_{P}\left[W_{t \mid n+1} F(B)_{t \mid n+1} \mid \mathcal{F}_{n}\right] \\
& =Q_{n}(t) E_{P}\left[r\left(A_{t \mid n+1}\right) \nu\left(B \mid A_{t \mid n+1}\right) 1_{A_{t \mid n+1} \neq \emptyset} \mid \mathcal{F}_{n}\right] \\
& =Q_{n}(t) \nu\left(B \mid A_{t \mid n}\right) 1_{A_{t \mid n} \neq \emptyset} \\
& =Q_{n}(t) F(B)_{t \mid n},
\end{aligned}
$$

where the first equality is measurability of $Q_{n}(t)$, the second is (7.7), and the third is Lemma 7.1. That is, ' $(\omega, t) \rightarrow Q_{n}(\omega, t) F(B)_{t \mid n}$ ' is a positive $T$-martingale and for each $\tau \in T^{*}, F(B)_{\tau}$ is $\sigma-\left\{W_{\tau \mid i}: i \leq n\right\}$-measurable and so $\mathbb{F}(B)$ is a weight system for the multiplicative cascade $\left\langle Q_{n}\right\rangle$.

A fundamental property of positive $T$-martingales $\left\langle Q_{n}\right\rangle_{n=0}^{\infty}$ is the following: if $q_{\emptyset}=E\left[Q_{0}\right] \in L^{1}(\mu)$ where $\mu \in M^{+}(T)$, then a.s. $\left\langle Q_{n} \mu\right\rangle_{n=0}^{\infty}$ converges weak* to a measure $Q_{\infty}(\mu)$, (where for each $n, Q_{n} \mu$ is the measure absolutely continuous with respect to $\mu$ with Radon-Nikodym derivative $Q_{n}$, and $Q_{\infty}$ is a random operator). See Kahane [9]. In the case $\mu=\lambda$, the following notation is adopted from [21] (with the exception of the use of $G_{-1}$ throughout this paper):

Definition 7.5. Let $\lambda$ denote normalized Haar measure on $T$. If $\mathbb{G}$ is a weight system for the multiplicative cascade $\left\langle Q_{n}\right\rangle_{n=0}^{\infty}$, and $G_{-1}=E\left[W_{\emptyset} G_{\emptyset}\right] \in L^{1}(\lambda)$, let $\lambda_{\mathbb{G}, n}$ denote the random measure $Q_{\mathbb{G}, n} \lambda$, and $\lambda_{\mathbb{G}, \infty}$ denote $Q_{\mathbb{G}, \infty} \lambda$. In the case $\mathbb{G}=\mathbf{1}\left(G_{\tau}=1\right.$ for all $\left.\tau\right)$, let $\lambda_{n}=Q_{n} \lambda$ and $\lambda_{\infty}=Q_{\infty}(\lambda)$. The measure valued process $\left\langle\lambda_{\mathbb{G}, n}\right\rangle_{n=0}^{\infty}$ is said to live if $\left\langle\lambda_{\mathbb{G}, n}(T)\right\rangle_{n}$ converges to $\lambda_{\mathbb{G}, \infty}(T)$ in $L^{1}(P)$. The process $\left\langle\lambda_{\mathbb{G}, n}\right\rangle_{n=0}^{\infty}$ living is equivalent to $E\left[\lambda_{\mathbb{G}, \infty}(T)\right]=G_{-1}$. The process $\left\langle\lambda_{\mathbb{G}, n}\right\rangle_{n=0}^{\infty}$ is said to die if $\lambda_{\mathbb{G}, n}(T)$ converges to 0 a.s.

We will also use the auxiliary measures $\mathcal{Q}_{\mathbb{G}}$ and $P_{\mathbb{G}, t}$ associated with the multiplicative cascade with weight system $\mathbb{G}, G_{-1} \neq 0$ as defined in section 2 of [21] (see statements 2.4 and Theorem 2.3 of that paper). We note a basic property of $P_{\mathbb{G}, t}$ here: for each bounded function $H: \Omega_{\left\langle\mathcal{P}_{n}\right\rangle_{n=0}^{\infty}} \rightarrow \mathbb{R}$ measurable with respect to $\sigma-\left\{W_{\tau}:|\tau| \leq n\right\}$,

$$
\int H d P_{\mathbb{G}, t}=G_{-1}^{-1} E_{P}\left[H \cdot \prod_{i=0}^{n} W_{t \mid i} G_{t \mid n}\right]
$$

The following lemma is a restatement of Corollaries 2.1, 2.3 and 2.4 of [21]:

Lemma 7.4. Let $\mathbf{G}$ be a weight system for the dependent cascade $\left\langle W_{\tau}\right\rangle_{\tau \in T^{*}}$ with $G_{-1}>0$, and let $a>0$. Then

(1) $\left\langle\lambda_{\mathbf{G}, n}\right\rangle_{n=0}^{\infty}$ lives if and only if for every bounded real valued Borel function $H$ on $\Omega_{\left\langle\mathcal{P}_{n}\right\rangle_{n=0}^{\infty}} \times T$

$$
\iint H(\omega, t) d \lambda_{\mathbf{G}, t} d P(\omega)=G_{-1} \iint H(\omega, t) d P_{\mathbf{G}, t}(\omega) d \lambda(t)
$$

(2) If $\lambda$-a.e.- $t, P_{\mathbf{G}, t}\left(\sum_{n=0}^{\infty} b^{-n} \prod_{i=0}^{n} W_{t \mid i} G_{t \mid i}<\infty\right)$, then $\left\langle\lambda_{\mathbf{G}, n}\right\rangle_{n=0}^{\infty}$ lives.

(3) If $\lambda$-a.e.-t, $P_{\mathbf{G}, t}\left(\limsup _{n \rightarrow \infty} b^{-n} \prod_{i=0}^{n} W_{t \mid i} G_{t \mid i}=\infty\right)$, then $\left\langle\lambda_{\mathbf{G}, n}\right\rangle_{n=0}^{\infty}$ dies.

(4) If $\lambda$-a.e.-t, $P_{\mathbf{G}, t}\left(\sum_{n=0}^{\infty} b^{a n} b^{-n} \prod_{i=0}^{n} W_{t \mid i} G_{t \mid i}<\infty\right)$, then $P$-a.s., $\lambda_{\mathbf{G}, \infty}$ gives each Borel set of dimension $\leq$ a measure zero.

(5) If $\lambda$-a.e.-t, $P_{\mathbf{G}, t}\left(\limsup _{n \rightarrow \infty} b^{a n} b^{-n} \prod_{i=0}^{n} W_{t \mid i} G_{t \mid i}=\infty\right)$, then $\lambda_{\mathbf{G}, \infty}$ is supported on a set of dimension $\leq a$. 
The collection of weight systems $\langle\mathbb{F}(B)\rangle_{B \in \mathcal{B}\left([0, d]^{\alpha}\right)}$ defined in Lemma 7.3 is 'differentiable' with respect to $\nu$ and has a local structure. See [21] for a definition of local structure.

Theorem 7.5. For each Borel $B$ of $[0, d]^{\alpha}$, let $\mathbb{F}(B)$ be defined as in Lemma 7.3 for a fixed $\kappa$-cascade-setup, $\kappa$ a basic multispectrum of spreading order $\geq 1$. Let $\nu$ be the spreading measure for $\kappa$. Then the following three statements hold:

(1) $\mathbb{F}(\cdot)$ has a local structure with respect to $\nu$ with a version of $\partial \mathbb{F}(\mathbf{s})$ given by

$$
\partial F(\mathbf{s})_{\tau}=\left\{\begin{array}{cc}
\nu\left(A_{\tau}\right)^{-1} 1_{\mathbf{s} \in A_{\tau}} & \text { if } A_{\tau} \neq \emptyset \\
0 & \text { if } A_{\tau}=\emptyset
\end{array}\right.
$$

for $\mathbf{s} \in[0, d]^{\alpha}$, and with respect to this choice version we have:

(2) For all $\mathbf{s} \in[0, d]^{\alpha}, \partial F(\mathbf{s})_{-1}=1$. For each $\mathbf{s} \in[0, d]^{\alpha}$, for all $t \in T$,

$$
P_{\partial F(\mathbf{s}), t^{-}} \text {a.s., for all } n, A_{t \mid n}=\mathcal{E}_{n}(\mathbf{s}) \text {. }
$$

(3) If $\sum_{n=0}^{\infty} b^{-n} \prod_{i=0}^{n} r\left(\mathcal{E}_{i}(\mathbf{s})\right) \nu\left(\mathcal{E}_{n}(\mathbf{s})\right)^{-1}<\infty$, then the process $\left\langle\lambda_{\partial \mathbb{F}(\mathbf{s}), n}\right\rangle_{n=0}^{\infty}$ lives.

Proof. Let $\mathbb{F}(\cdot)$ be the measure valued weight decomposition defined by $F_{\tau}(B)=$ $\nu\left(B \mid A_{\tau}\right) \cdot 1_{A_{\tau} \neq \emptyset}$. When $A_{\tau} \neq \emptyset, \nu\left(B \mid A_{\tau}\right) \cdot 1_{A_{\tau} \neq \emptyset}=\nu\left(A_{\tau}\right)^{-1} \int_{A_{\tau}} 1_{B} d \nu$ so $(1)$ follows.

For any $\mathbf{s} \in[0, d]^{\alpha}, \partial F(\mathbf{s})_{-1}=E\left[W_{\emptyset} \nu\left(A_{\emptyset}\right)^{-1} 1_{A_{\emptyset}}(\mathbf{s})\right]=r\left([0, d]^{\alpha}\right) \cdot P\left(A_{\emptyset}=[0, d]^{\alpha}\right)$ $=1$. Fix $t \in T$. By statement (7.8),

$$
P_{\partial \mathbb{F}(\mathbf{s}), t}\left(A_{t \mid n}=\mathcal{E}_{n}(\mathbf{s})\right)=\partial F(\mathbf{s})_{-1}^{-1} E_{P}\left(\prod_{i=0}^{n} W_{t \mid n} \partial F(\mathbf{s})_{t \mid n} 1_{A_{t \mid n}=\mathcal{E}_{n}(\mathbf{s})}\right) .
$$

Since $1_{A_{t \mid n}=\mathcal{E}_{n}(\mathbf{s})}=1_{\mathbf{s} \in A_{t \mid n}},(7.9)$ implies

$$
\partial F(\mathbf{s})_{t \mid n} 1_{A_{t \mid n}=\mathcal{E}_{n}(\mathbf{s})}=\partial F(\mathbf{s})_{t \mid n} .
$$

Applying (7.11) to the right hand side of (7.10), we have $P_{\partial \mathbb{F}(\mathbf{s}), t}\left(A_{t \mid n}=\mathcal{E}_{n}(\mathbf{s})\right)=$ $\partial F(\mathbf{s})_{-1}^{-1} E_{P}\left(\prod_{i=0}^{n} W_{t \mid n} \partial F(\mathbf{s})_{t \mid n}\right)=\partial F(\mathbf{s})_{-1}^{-1} \partial F(\mathbf{s})_{-1}=1$. Thus (2) follows.

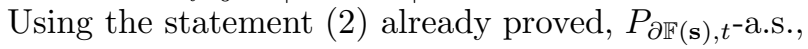

$$
\sum_{n=0}^{\infty} b^{-n} \prod_{i=0}^{n} W_{t \mid i} \partial F(\mathbf{s})_{t \mid n}=\sum_{n=0}^{\infty} b^{-n} \prod_{i=0}^{n} r\left(\mathcal{E}_{i}(\mathbf{s})\right) \nu\left(\mathcal{E}_{n}(\mathbf{s})\right)^{-1} .
$$

Now apply Lemma 7.4 part (2).

Lemma 7.6. Within the setup of Theorem 7.5, let $\mathcal{L}=\left\{\mathbf{s} \in[0, d]^{\alpha}:\left\langle\lambda_{\partial \mathbb{F}(\mathbf{s}), n}\right\rangle_{n=0}^{\infty}\right.$ lives\}.

(1) If $\zeta$ is a finite measure on $\mathcal{B}(\mathcal{L})$, then $\int \partial \mathbb{F} d \zeta \stackrel{\text { def }}{=}\left\langle\int \partial F(\mathbf{s})_{\tau} d \zeta(\mathbf{s})\right\rangle_{\tau \in T^{*}}$ defines a weight system. Further, $\left\langle\lambda_{\int \partial \mathbb{F} d \zeta, n}\right\rangle_{n=0}^{\infty}$ lives and $P$-a.s.

$$
\lambda_{\int \partial \mathbb{F} d \zeta, \infty}=w^{*}-\int \lambda_{\partial \mathbb{F}(\mathbf{s}), \infty} d \zeta(\mathbf{s}) .
$$

If $B$ is a $\zeta$-measurable subset of $\mathcal{L}$ with $\zeta(B)=\zeta(\mathcal{L})$, then $\lambda$-a.e.-t, $P_{\int} \partial \mathbb{F} d \zeta, t^{-}$ a.s., there is a (random) $\mathbf{s} \in B$ so that $A_{t \mid n}=\mathcal{E}_{n}(\mathbf{s})$ for all $n$. 
(2) Suppose $\zeta$ is a finite measure supported on the set

$$
\left\{\mathbf{s} \in \mathcal{L}: d-d \cdot \lim _{n \rightarrow \infty} \log _{b} r\left(\mathcal{E}_{n}(\mathbf{s})\right)=s_{0}\right\}
$$

Let $\varphi:[0, d]^{\alpha} \rightarrow \mathbf{R}$ be defined by $\varphi(\mathbf{s})=s_{0}-d \cdot \limsup _{n \rightarrow \infty} \frac{1}{n} \log _{b} \frac{\zeta\left(\mathcal{E}_{n}(\mathbf{s})\right)}{\nu\left(\mathcal{E}_{n}(\mathbf{s})\right)}$. Then P-a.s.

$$
K\left(\lambda_{\int \partial \mathbb{F} d \zeta, \infty}\right)=w^{*}-\int \lambda_{\partial \mathbb{F}(\mathbf{s}), \infty} \times \delta_{\varphi(\mathbf{s})} d \zeta(\mathbf{s})
$$

Proof. That $\int \partial \mathbb{F} d \zeta$ is a weight system follows from Fubini's Theorem. Let $\mathbf{s} \in \mathcal{L}$. Since $\left\langle\lambda_{\partial \mathbb{F}(\mathbf{s}), n}\right\rangle_{n=0}^{\infty}$ lives, Corollary 2.2. of [21] implies

$$
E\left[\lambda_{\partial \mathbb{F}(\mathbf{s}), \infty}\left(\left\{t: \forall n, A_{t \mid n}=\mathcal{E}_{n}(\mathbf{s})\right\}\right)\right]=\int P_{\partial \mathbb{F}(\mathbf{s})}\left(\forall n, A_{t \mid n}=\mathcal{E}_{n}(\mathbf{s})\right) d \lambda(t) .
$$

This together with part (2) of Theorem 7.5 implies

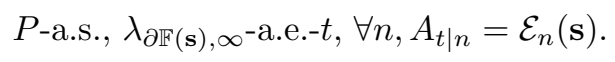

Since $\zeta$ is supported on $\mathcal{L}$, Theorem 2.11 of $[21]$ implies $\left\langle\lambda_{\int \partial \mathbb{F} d \zeta, n}\right\rangle_{n=0}^{\infty}$ lives, and $P$-a.s.

$$
\lambda_{\int \partial \mathbb{F} d \zeta, \infty}=w^{*}-\int \lambda_{\partial \mathbb{F}(\mathbf{s}), \infty} d \zeta(\mathbf{s})
$$

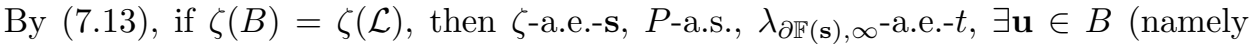
s) so that $\forall n, A_{t \mid n}=\mathcal{E}_{n}(\mathbf{u})$. Using Fubini's Theorem and (7.14), the preceding statement becomes $P$-a.s., $\lambda_{\int \partial \mathbb{F} d \zeta, \infty}$-a.e.- $t, \exists \mathbf{u} \in B$ so that $\forall n, A_{t \mid n}=\mathcal{E}_{n}(\mathbf{u})$. But since $\left\langle\lambda_{\int \partial \mathbb{F} d \zeta, n}\right\rangle_{n=0}^{\infty}$ lives, Theorem 7.5 part (1) and this last implies $\lambda$-a.e.- $t$, $P_{\int \partial \mathbb{F} d \zeta, t^{- \text {a.s. }}}$, there is a (random) $\mathbf{s} \in B$ so that $A_{t \mid n}=\mathcal{E}_{n}(\mathbf{s})$ for all $n$.

For part (2), note that the function $\varphi$ is Borel. If $a, b$ are extended reals with $a<b$, then

$$
\mathbb{G}(a, b)=\int 1_{(a, b)} \circ \varphi(\mathbf{s}) \partial \mathbb{F}(\mathbf{s}) d \zeta(\mathbf{s})
$$

defines a weight system by part (1). Let

$$
C=\left\{\mathbf{s} \in \varphi^{-1}(a, b): \frac{\zeta\left(\mathcal{E}_{n}(\mathbf{s}) \cap \varphi^{-1}(a, b)\right)}{\zeta\left(\mathcal{E}_{n}(\mathbf{s})\right)} \rightarrow 1 \text { as } n \rightarrow \infty\right\} .
$$

Easily by the definition of $\varphi$, if $\mathbf{s} \in C$,

$$
\limsup _{n \rightarrow \infty} \frac{1}{n} \frac{\zeta\left(\mathcal{E}_{n}(\mathbf{s}) \cap \varphi^{-1}(a, b)\right)}{\nu\left(\mathcal{E}_{n}(\mathbf{s})\right)}=d^{-1}\left(s_{0}-\varphi(\mathbf{s})\right) .
$$

The Levi 0-1 law implies $\zeta(C)=\zeta\left(\varphi^{-1}(a, b)\right)$. By part $(1), \lambda$-a.e.- $t, P_{\mathbb{G}, t \text {-a.s., there }}$ is a (random) $\mathbf{s} \in B$ so that $A_{t \mid n}=\mathcal{E}_{n}(\mathbf{s})$ for all $n$. Continuing with this same $\mathbf{s}$, using (7.12) and (7.15),

$$
\limsup _{n \rightarrow \infty} \frac{1}{n} \log _{b} b^{(-1+c) n}\left(\prod_{i=0}^{n} W_{t \mid i}\right) G(a, b)_{t \mid n}=c-d^{-1} \cdot \varphi(\mathbf{s}) .
$$


By Lemma 7.4 parts (4) and (5), $P$-a.s., $\lambda_{\mathbb{G}(a, b), \infty}$ is supported on a set of dimension $\leq b$ and gives sets of dimension $<a$ measure zero. If $\zeta(\{x\})=0$, then equation (7.14) implies

$$
\lambda_{\mathbb{G}(-\infty, x), \infty}+\lambda_{\mathbb{G}(x, \infty), \infty}=w^{*}-\int \lambda_{\partial \mathbb{F}(\mathbf{s}), \infty} d \zeta(\mathbf{s})=\lambda_{\int \partial \mathbb{F}(\mathbf{s}), \infty} d \zeta(\mathbf{s}) .
$$

The last two statements together with Theorem 2.2 imply for $\zeta(\{x\})=0$,

$$
K\left(\lambda_{\int \partial \mathbb{F} d \zeta, \infty}\right) 1_{T \times[0, x]}=\lambda_{\mathbb{G}(-\infty, x], \infty}=w^{*}-\int_{[0, x]} \lambda_{\partial \mathbb{F}(\mathbf{s}), \infty} \times \delta_{\varphi(\mathbf{s})} d \zeta(\mathbf{s}) .
$$

Since there exists a countable dense set $D$ of reals so that $\zeta \circ \varphi^{-1}(D)=0$ and the cumulative mass distribution of the form of the left hand side of (7.16) are right continuous, it follows that $P$-a.s., for all $x$, the equation $(7.16)$ holds.

Theorem 7.7. Let $\kappa$ be a basic multispectrum of order $\alpha \geq 1$ with dimension spreading measure $\nu$ and unidimensional map $U:[0, d]^{\alpha} \rightarrow[0, \infty]$. Suppose that $\left\langle\left\langle\mathcal{P}_{n}\right\rangle_{n=0}^{\infty}, r\right\rangle$ is a coordinate induction pair for $\kappa$ so that $\nu$-a.e.-s,

$$
\sum_{n=0}^{\infty} b^{-n} \prod_{i=0}^{n} r\left(\mathcal{E}_{i}(\mathbf{s})\right) \nu\left(\mathcal{E}_{n}(\mathbf{s})\right)^{-1}<\infty
$$

Let $P$ be the cascade measure generated by the coordinate induction pair. Then the measure valued process $\left\langle\lambda_{n}\right\rangle_{n=0}^{\infty}$ lives with respect to $P$ and

$$
P \text {-a.s., } \sigma_{\omega_{1}}\left(\lambda_{\infty}\right) \ll \kappa .
$$

Further, if $B$ is a Borel subset of $[0, \infty]^{\omega_{1}}$ with $\kappa(B)>0$, then $P\left(\sigma_{\omega_{1}}\left(\lambda_{\infty}\right)(B)>\right.$ 0) $>0$.

Proof. Let $\mathcal{L}$ be as Lemma 7.6. Theorem 7.5 part (3) and (7.17) implies $\nu(\mathcal{L})=1$.

Let Ind $=\left\{\mathbf{s} \in[0, d]^{\alpha}: s_{0}-d \cdot \limsup _{n \rightarrow \infty} \frac{1}{n} \log _{b}\left(\frac{\nu\left(\mathcal{E}_{n}(\mathbf{s})\left|\theta_{\alpha, \beta}=\mathbf{s}\right|_{\beta}\right)}{\nu\left(\mathcal{E}_{n}(\mathbf{s})\right)}\right)=s_{\beta}\right.$ and $s_{0}-d \cdot \limsup _{n \rightarrow \infty} \frac{1}{n} \log _{b}\left(\frac{1}{\nu\left(\mathcal{E}_{n}(\mathbf{s})\right)}\right)=U(\mathbf{s})$, and $\left.d-d \cdot \lim _{n \rightarrow \infty} \log _{b}\left(r\left(\mathcal{E}_{n}(\mathbf{s})\right)\right)=s_{0}\right\}$. Since $\left\langle\left\langle\mathcal{P}_{n}\right\rangle_{n=0}^{\infty}, r\right\rangle$ is a coordinate induction pair, $\nu($ Ind $)=1$.

Fix $\mathbf{s} \in \mathcal{L} \cap$ Ind. Letting $\zeta=\delta_{\mathbf{s}}$ in Lemma 7.6 part (2), the $\varphi$ for $\delta_{\mathbf{s}}$ satisfies

$$
\varphi(\mathbf{s})=s_{0}-d \cdot \limsup _{n \rightarrow \infty} \frac{1}{n} \log _{b}\left(\frac{1}{\nu\left(\mathcal{E}_{n}(\mathbf{s})\right)}\right)=U(\mathbf{s}) .
$$

This implies by Lemma 7.6 part (2), P-a.s., $\sigma\left(\lambda_{\partial \mathbb{F}(\mathbf{s}), \infty)}\right) \ll \zeta \circ \varphi^{-1}=\delta_{U(\mathbf{s})}$. Thus $P$-a.s., $\nu$-a.e.-s, if $\lambda_{\partial \mathbb{F}(\mathbf{s}), \infty} \neq 0$, then $\lambda_{\partial \mathbb{F}(\mathbf{s}), \infty)}$ is unidimensional of dimension $U(\mathbf{s})$.

For each $\beta<\alpha$, let $\left\langle\nu_{\theta_{\alpha, \beta}=\mathbf{u}}\right\rangle_{\mathbf{u} \in[0, d]^{\beta}}$ be the regular conditional expectation of $\nu$ with respect to the map $\theta_{\alpha, \beta}$. Since $\nu(\mathcal{L})=1$, for $\nu \circ \theta_{\alpha, \beta}^{-1}$-a.e.-u, $\nu_{\theta_{\alpha, \beta}=\mathbf{u}}(\mathcal{L})=1$.

Claim $\zeta$ : $P$-a.s. the following two conditions hold:

(0) $K\left(\lambda_{\infty}\right)=w^{*}-\int \lambda_{\partial \mathbb{F}(\mathbf{s}), \infty} \times \delta_{s_{0}} d \nu(\mathbf{s})$ and

(1) for $1 \leq \beta<\alpha, \nu \circ \theta_{\alpha, \beta}^{-1}$-a.e.-u,

$$
K\left(w^{*}-\int \lambda_{\partial \mathbb{F}(\mathbf{s}), \infty} d \nu_{\theta_{\alpha, \beta}=\mathbf{u}}(\mathbf{s})\right)=w^{*}-\int \lambda_{\partial \mathbb{F}(\mathbf{s}), \infty} \times \delta_{s_{\beta}} d \nu_{\theta_{\alpha, \beta}=\mathbf{u}}(\mathbf{s}) .
$$


Using again part (2) of Lemma 7.6, (0) follows from the calculation: for $\zeta=\nu$, $\varphi(\mathbf{s})=s_{0}-d \cdot \limsup \frac{1}{n} \log _{b}\left(\frac{\nu\left(\mathcal{E}_{n}(\sigma)\right)}{\nu\left(\mathcal{E}_{n}(\sigma)\right)}\right)=s_{0}$. Similarly for $(1)$, since $\nu($ Ind $)=1$, for $\nu \circ \theta_{\alpha, \beta^{-a}}^{-1 . e .-u}$, if $\zeta=\nu_{\theta_{\alpha, \beta}=\mathbf{u}}$, then

$$
\varphi(\mathbf{s})=s_{0}-d \cdot \limsup _{n \rightarrow \infty} \frac{1}{n} \log _{b}\left(\frac{\nu\left(\mathcal{E}_{n}(\mathbf{s})\left|\theta_{\alpha, \beta}=\mathbf{s}\right|_{\beta}\right)}{\nu\left(\mathcal{E}_{n}(\mathbf{s})\right)}\right)=s_{\beta} .
$$

Thus Claim $\zeta$ holds.

Claim $\kappa$ : P-a.s., $\lambda_{\infty, \omega_{1}}=w^{*}-\int \lambda_{\partial \mathbb{F}\left(\theta_{\omega_{1}, \alpha}(\mathbf{s})\right), \infty} \times \delta_{\mathbf{s}} d \kappa(\mathbf{s})$.

To establish this claim it is enough to show $P$-a.e., $\lambda_{\infty, \alpha}=w^{*}-\int \lambda_{\partial \mathbb{F}(\mathbf{s}), \infty} \times$ $\delta_{\mathbf{s}} d \nu(\mathbf{s})$, since this and the fact $P$-a.s., $\nu$-a.e.-s, $\lambda_{\partial \mathbb{F}(\mathbf{s}), \infty}$ is unidimensional of dimension $U(\mathbf{s})$, will imply the claim. But this follows from Claim $\zeta$ and Theorem 6.7 and the fact that $P$-a.s., $\lambda_{\infty}=w^{*}-\int \lambda_{\partial \mathbb{F}(\mathbf{s}), \infty} d \nu(\mathbf{s})$.

Claim $\kappa$ implies $\sigma_{\omega_{1}}\left(\lambda_{\infty}\right) \ll \kappa P$-a.s. And, for $B \in \mathcal{B}\left([0, \infty]^{\omega_{1}}\right), E_{P}\left[\sigma_{\omega_{1}}\left(\lambda_{\infty}\right)(B)\right]$ $=E_{P}\left[\int_{B} \lambda_{\partial \mathbb{F}\left(\theta_{\omega_{1}, \alpha}(\mathbf{s})\right), \infty}(T) d \kappa(\mathbf{s})\right]=\int_{B} \partial \mathbb{F}_{-1}\left(\theta_{\omega_{1}, \alpha}(\mathbf{s})\right) d \kappa(\mathbf{s})=\kappa(B)$, where the first equality comes from Claim $\kappa$, the second by Fubini's Theorem, and the third since $\partial \mathbb{F}_{-1}\left(\theta_{\omega_{1}, \alpha}(\mathbf{s})\right)=1$. Thus the last statement of the theorem follows.

Remark 7.1. Note that $P$-a.s., the Borel map $\phi_{\lambda_{\infty}, \omega_{1}}$ associated with $\lambda_{\infty}$ is defined $\lambda_{\infty}$-a.e. by the formula $\phi_{\lambda_{\infty}, \omega_{1}}(t)=\mathbf{s} * \hat{U}(\mathbf{s})$ where $\mathbf{s}$ is the unique element of Ind so that $A_{t \mid n}=\mathcal{E}_{n}(\mathbf{s})$ for all $n$, i.e., $\bigcap A_{t \mid n}=\left\{\phi_{\lambda_{\infty}, \omega_{1}}(t)\right\}$ for $\lambda_{\infty}$-a.e.-t, $P$-a.s.

\section{Existence of Positive Filtration Bases}

The existence of positive filtration bases for basic multispectrums which encode induction rules needs to be demonstrated. The following $\kappa$-setup will be assumed throughout this section:

$\kappa$-Setup: Let $\kappa$ be a basic multispectrum of diffuseness order $\alpha \geq 1$, with spreading measure $\nu$ and unidimensional map $U$. We suppose $\kappa$-a.e.-s, $s_{0} \leq d$, and $T=\{0,1, \ldots, b-1\}^{\mathbb{N}}$, as in Section 7.

Definition 8.1. For each $\gamma<\alpha$, let $S_{\gamma}:[0, d]^{\alpha} \rightarrow[0, d]$ be the coordinate projection defined by $S_{\gamma}\left(\left\langle s_{\beta}\right\rangle_{\beta<\alpha}\right)=s_{\gamma}$. Set $S_{\alpha}=U$, the unidimensional map of $\kappa . E_{\nu}$ is the expectation operator with respect to the spreading measure $\nu$. For each $\gamma<\alpha$ and $\mathbf{s} \in M^{+}\left([0, d]^{\alpha}\right)$, let $H_{\gamma}(\mathbf{s})=\nu\left(S_{\gamma}^{-1}\left[0, s_{\gamma}\right]\left|\theta_{\alpha, \gamma}=\mathbf{s}\right|_{\gamma}\right)$ where $\left.\mathbf{s}\right|_{\gamma}$ is the truncation $\theta_{\alpha, \gamma}(\mathbf{s})$. The map $H_{\gamma}:[0, d]^{\alpha} \rightarrow[0,1]$ thereby indicated is $\nu$-essentially defined and $\nu$-measurable. Define $\mathcal{H}:[0, d]^{\alpha} \rightarrow[0,1]^{\alpha}$ by $\mathcal{H}(\mathbf{s})=\left\langle H_{\gamma}(\mathbf{s})\right\rangle_{\gamma<\alpha}$. For $\mathbf{u} \in[0, d]^{\beta}$, let $H_{\beta}^{\prime}(\cdot \mid \mathbf{u})$ denote the cumulative distribution function of $\nu\left(S_{\beta}^{-1}(\cdot) \mid \theta_{\alpha, \beta}=u\right)$.

Lemma 8.1. If $0=\gamma_{1}<\gamma_{2}<\cdots<\gamma_{m+1}=\alpha$, then $\nu$-a.s.,

$$
S_{\gamma_{i}}>S_{\gamma_{i+1}} \text { if } 0<i<m-1 \text {, }
$$

and

$$
S_{\gamma_{m}} \geq U=S_{\gamma_{m+1}} .
$$

Proof. This is an easy consequence of the definition of basic multispectrum of spreading order $\alpha \geq 1$.

Definition 8.2. Let $F$ be a function from the set $X$ to the set $Y$, and $A \subseteq X$. The set $A$ is said to be saturated with respect to $F$ if and only if $A=F^{-1}(F(A))$. 
Lemma 8.2. For $\beta<\alpha, \nu \circ \theta_{\alpha, \beta}^{-1}$-a.e.-u,

$$
\nu_{\theta_{\alpha, \beta=\mathbf{u}}}\left\{\mathbf{s}: \exists \mathbf{v} \text { so that }\left.\mathbf{v}\right|_{\beta}=\left.\mathbf{s}\right|_{\beta} \text { and } v_{\beta} \neq s_{\beta} \text { but } H_{\beta}(\mathbf{v})=H_{\beta}(\mathbf{s})\right\}=0
$$

and

$$
\nu_{\theta_{\alpha, \beta=\mathbf{u}}} \circ H_{\beta}^{-1}=\lambda
$$

where $\left\langle\nu_{\theta_{\alpha, \beta=\mathbf{u}}}\right\rangle_{\mathbf{u} \in[0, d]^{\beta}}$ is the regular conditional probability of $\nu$ with respect to $\theta_{\alpha, \beta}$.

Proof. It is well known that if $P$ is a non-atomic probability measure on the Borel sets of $\mathbb{R}$ with cumulative distribution function $F$, then

$$
P \circ F^{-1}=\lambda,
$$

and

$$
P(\{x: \exists y \neq x \text { s.t. } F(x)=F(y)\})=0 .
$$

Let $A$ be a universally measurable subset of $[0, d]^{\beta} \times \mathbb{R}$, and for each $\mathbf{u} \in[0, d]^{\beta}$, let $A(\mathbf{u})=\{x \in \mathbb{R}:\langle\mathbf{u}, x\rangle \in A\}$. For $\nu \circ \theta_{\alpha, \beta^{-}}^{-1}$.e.- $\mathbf{u} \in[0, d]^{\beta}$,

$$
\begin{aligned}
\nu_{\theta_{\alpha, \beta=\mathbf{u}}} \circ H_{\beta}^{-1}(A(\mathbf{u})) & =\nu_{\theta_{\alpha, \beta=\mathbf{u}}}\left(\left\{\mathbf{s} \in[0, d]^{\alpha}: H_{\beta}(\mathbf{s}) \in A(\mathbf{u})\right\}\right) \\
& =\nu_{\theta_{\alpha, \beta=\mathbf{u}}}\left(\left\{\mathbf{s} \in[0, d]^{\alpha}: H_{\beta}^{\prime}\left(s_{\beta}|\mathbf{s}|_{\beta}\right) \in A(\mathbf{u})\right\}\right) \\
& =\nu_{\theta_{\alpha, \beta=\mathbf{u}}}\left(\left\{\mathbf{s} \in[0, d]^{\alpha}: H_{\beta}^{\prime}\left(s_{\beta} \mid \mathbf{u}\right) \in A(\mathbf{u})\right\}\right) \\
& =\nu_{\theta_{\alpha, \beta=\mathbf{u}}} \circ S_{\beta}^{-1}\left(\left\{y: H_{\beta}^{\prime}(y \mid \mathbf{u}) \in A(\mathbf{u})\right\}\right) .
\end{aligned}
$$

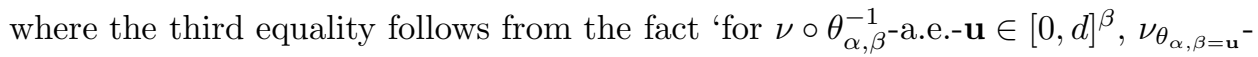
a.e.-s $\in[0, d]^{\alpha},\left.\mathbf{s}\right|_{\beta}=\mathbf{u}^{\prime}$. Since $\kappa$ is a basic multispectrum of order $\alpha$ and $\beta<\alpha$, we have $\nu \circ \theta_{\alpha, \beta}^{-1}$-a.e.-u $\in[0, d]^{\beta}, \nu_{\theta_{\alpha, \beta=\mathbf{u}}} \circ S_{\beta}^{-1}$ is non-atomic. Thus, (8.7) and (8.5) together imply (8.4).

For statement 8.3, note $\nu_{\theta_{\alpha, \beta=\mathbf{u}}}\left\{\mathbf{s}: \exists \mathbf{v}\right.$ so that $\left.\mathbf{v}\right|_{\beta}=\left.\mathbf{s}\right|_{\beta}$ and $v_{\beta} \neq s_{\beta}$ but $\left.H_{\beta}(\mathbf{v})=H_{\beta}(\mathbf{s})\right\}=\nu_{\theta_{\alpha, \beta=\mathbf{u}}}\left\{\mathbf{s}: \mathbf{u}=\left.\mathbf{s}\right|_{\beta}\right.$ and $\exists x$ so that $x \neq s_{\beta}$ but $H_{\beta}^{\prime}\left(s_{\beta} \mid \mathbf{u}\right)=$ $\left.H_{\beta}^{\prime}(x \mid \mathbf{u})\right\}$ since $\mathbf{u}=\left.\mathbf{s}\right|_{\beta}, \nu_{\theta_{\alpha, \beta}=\mathbf{u}}$-a.s. Letting $A(\mathbf{u})=\left\{H_{\beta}^{\prime}(x \mid \mathbf{u}) \in \mathbb{R}: \exists z \neq x\right.$ so that $\left.H_{\beta}^{\prime}(z \mid \mathbf{u})=H_{\beta}^{\prime}(x \mid \mathbf{u})\right\},{ }^{6}$ and applying (8.7) and the preceding sentence, we have $\nu_{\theta_{\alpha, \beta}=\mathbf{u}}\left\{\mathbf{s}: \exists \mathbf{v}\right.$ so that $\left.\mathbf{v}\right|_{\beta}=\left.\mathbf{s}\right|_{\beta}$ and $v_{\beta} \neq s_{\beta}$ but $\left.H_{\beta}(\mathbf{v})=H_{\beta}(\mathbf{s})\right\}=$ $\nu_{\theta_{\alpha, \beta=\mathbf{u}}} \circ S_{\beta}^{-1}\left\{y: H_{\beta}^{\prime}(y \mid \mathbf{u}) \in A(\mathbf{u})\right\}$. Applying (8.6) to the last equation implies (8.3).

Lemma 8.3. The $\sigma$-algebra, $\sigma(\mathcal{H})$, is almost the $\nu$-measurable sets. Further, for each $1 \leq \beta \leq \alpha, \sigma-\left(H_{\gamma} ; \gamma<\beta\right)$ is $\nu$-almost $\sigma-\left(S_{\gamma} ; \gamma<\beta\right)=\sigma-\left(\theta_{\alpha, \beta}\right)$.

Proof. The first statement will follow if the second statement can be proved. For $\gamma<\alpha$, we fix the functions $H_{\gamma}$ to be Borel and to be measurable with respect to $\theta_{\alpha, \gamma+1}$ by changing them, if necessary, with respect to countably many sets of measure zero. Thus $\sigma-\left(H_{\gamma} ; \gamma<\beta\right)$ is contained in the $\sigma$-algebra generated by $\sigma-\left(S_{\gamma} ; \gamma<\beta\right)$. Let $\mathbf{H}_{\beta}=\left\langle H_{\gamma}\right\rangle_{\gamma<\beta}$. For each $\beta \leq \alpha$, let

$A_{\beta}=\left\{\mathbf{s} \in[0, d]^{\alpha}: \exists \mathbf{u} \in[0, d]^{\alpha}\right.$ so that $\left.\mathbf{s}\right|_{\beta} \neq\left.\mathbf{u}\right|_{\beta}$ but $H_{\gamma}(\mathbf{s})=H_{\gamma}(\mathbf{u})$ for $\left.\gamma<\beta\right\}$.

We will show that $\nu\left(A_{\beta}\right)=0$ for all $\beta \leq \alpha$. Easily $A_{0}=\emptyset$, and so $\nu\left(A_{0}\right)=0$.

\footnotetext{
${ }^{6}$ Obviously, $A=\left\{\left\langle\mathbf{u}, H_{\beta}^{\prime}(x \mid \mathbf{u})\right\rangle: \exists z \neq x\right.$ so that $\left.H_{\beta}^{\prime}(z \mid \mathbf{u})=H_{\beta}^{\prime}(x \mid \mathbf{u})\right\}$ is coanalytic if $H_{\beta}^{\prime}$ is chosen to be Borel.
} 
Suppose $\nu\left(A_{\gamma}\right)=0$ for all $\gamma<\beta$. If $\beta$ is a limit ordinal, then $A_{\beta} \cap \bigcap_{\gamma<\beta} A_{\gamma}^{c}=\emptyset$ and so $\nu\left(A_{\beta}\right)=0$. If $\beta=\gamma+1$, then $A_{\beta} \cap A_{\gamma}^{c}=\left\{\mathbf{s}: \exists \mathbf{u}\right.$ so that $\left.\mathbf{u}\right|_{\gamma}=\left.\mathbf{s}\right|_{\gamma}$ and $u_{\gamma} \neq s_{\gamma}$ but $\left.H_{\gamma}(\mathbf{u})=H_{\gamma}(\mathbf{s})\right\}$. By Lemma 8.2 statement $(8.3), \nu\left(A_{\beta}\right)=0$.

Set $B_{\beta}=[0, d]^{\alpha} \backslash \bigcup_{\gamma: \gamma<\beta} A_{\gamma}$. Easily, $B_{\beta}=\left\{\mathbf{s} \in[0, d]^{\alpha}: \forall \mathbf{u} \in[0, d]^{\alpha}\right.$, if $\forall \gamma<\beta$, $H_{\gamma}(\mathbf{s})=H_{\gamma}(\mathbf{u})$, then $\left.\theta_{\alpha, \beta}(\mathbf{s})=\theta_{\alpha, \beta}(\mathbf{u})\right\}$. Now, $\nu\left(B_{\beta}\right)=1$, and since $H_{\gamma}$ is measurable with respect to $\theta_{\alpha, \beta}$ for $\gamma<\beta, B_{\beta}$ is saturated with respect to the map $\theta_{\alpha, \beta}$. Let $C$ be a Borel subset of $[0, d]^{\alpha}$ which is saturated with respect to $\theta_{\alpha, \beta}{ }^{7}$ and $C \subseteq B_{\beta}$. Since $C \subseteq B_{\beta}$ and $C$ is saturated with respect to $\theta_{\alpha, \beta}, C$ is saturated with respect to $\mathbf{H}_{\beta}$ :

$$
\mathbf{H}_{\beta}^{-1}\left(\mathbf{H}_{\beta}(C)\right)=C .
$$

Since $\mathbf{H}_{\beta}(C)$ is analytic, there is a Borel $D \subseteq \mathbf{H}_{\beta}(C)$ so that $\nu\left(\mathbf{H}_{\beta}^{-1}(D)\right)=$ $\nu\left(\mathbf{H}_{\beta}^{-1}\left(\mathbf{H}_{\beta}(C)\right)\right)$. Since $\mathbf{H}_{\beta}^{-1}(D)$ is in $\sigma-\left(H_{\gamma} ; \gamma<\beta\right), C$ is in the $\sigma$-algebra generated by the $\nu$-null sets and $\sigma\left(H_{\gamma} ; \gamma<\beta\right)$. It follows that $\sigma-\left(S_{\gamma} ; \gamma<\beta\right)$ is a subset of in the $\sigma$-algebra generated by the $\nu$-null sets and $\sigma\left(H_{\gamma} ; \gamma<\beta\right)$.

Lemma 8.4. $\nu \circ \mathcal{H}^{-1}=\lambda^{\alpha}$ where $\lambda$ is Lebesgue measure on $[0,1]$.

Proof. It is enough to show that

$$
\nu \circ \mathcal{H}^{-1} \circ \theta_{\alpha, \beta}^{-1}=\lambda^{\beta}
$$

for all $1 \leq \beta \leq \alpha$. Now, $\mathbf{H}_{\beta}=\left\langle H_{\gamma}\right\rangle_{\gamma<\beta}$ is $\theta_{\alpha, \beta}$-measurable and so there is a measurable function $G:[0, d]^{\beta} \rightarrow[0, d]^{\beta}$ so that $\left\langle H_{\gamma}(\mathbf{s})\right\rangle_{\gamma<\beta}=G\left(\theta_{\alpha, \beta}(\mathbf{s})\right)$. For any bounded measurable $g:[0, d]^{\beta+1} \rightarrow \mathbb{R}$,

$$
\begin{aligned}
\int g d \nu \circ \mathcal{H}^{-1} \circ \theta_{\alpha, \beta+1}^{-1} & =\int g\left(\left\langle H_{\gamma}(\mathbf{s})\right\rangle_{\gamma<\beta+1}\right) d \nu(\mathbf{s}) \\
& =\iint g\left(G(\mathbf{u}) * H_{\beta}(\mathbf{s})\right) d \nu_{\theta_{\alpha, \beta} \mathbf{u}}(\mathbf{s}) d \nu \circ \theta_{\alpha, \beta}^{-1}(\mathbf{u}) \\
& =\iint g(G(\mathbf{u}) * x) d \nu_{\theta_{\alpha, \beta=\mathbf{u}}} \circ H_{\beta}^{-1}(x) d \nu \circ \theta_{\alpha, \beta}^{-1}(\mathbf{u}) \\
& =\iint g(G(\mathbf{u}) * x) d \lambda(x) d \nu \circ \theta_{\alpha, \beta}^{-1}(\mathbf{u}) \\
& =\iint g\left(\left\langle H_{\gamma}(\mathbf{s})\right\rangle_{\gamma<\beta} * x\right) d \lambda(x) d \nu(\mathbf{s})
\end{aligned}
$$

where the fourth equality is Lemma 8.2 statement (8.5). Using the uniqueness of Kolmogoroff extensions and the above, statement (8.8) follows by an easy induction.

Definition 8.3. For any $\gamma<\alpha$ and Borel $B \subseteq[0,1]$, define

$$
F_{\gamma}(B)=\mathcal{H}^{-1}\left\{\mathbf{s} \in[0,1]^{\alpha}: s_{\gamma} \in B\right\} .
$$

For each $\gamma<\alpha$, let $\mathcal{F}_{=\gamma}$ be the $\sigma$-field $\left\{F_{\gamma}(B): B\right.$ is a Borel subset of $\left.[0,1]\right\}$. A subset $A$ of $[0, d]^{\alpha}$ is called a multi- $b^{k}$-tile if there exists a finite subset $D$ of $\alpha$, a

\footnotetext{
${ }^{7}$ The $\sigma$-algebra generated by $\theta_{\alpha, \beta}$ consists precisely of the Borel subsets of $[0, d]^{\alpha}$ which are saturated with respect to $\theta_{\alpha, \beta}$, since $\theta_{\alpha, \beta}$ is the projection of the product of two Polish spaces onto the first factor.
} 
finite sequence, $\left\langle k_{\gamma}\right\rangle_{\gamma \in D}$, of natural numbers indexed by elements of $D$, and a finite sequence, $\left\langle i_{\gamma}\right\rangle_{\gamma \in D}$, of numbers with $i_{\gamma} \in\left\{1,2, \ldots, b^{k_{\gamma}}\right\}$ for each $\gamma \in D$ so that

$$
A=\bigcap_{\gamma \in D} F_{\gamma}\left(I_{\gamma}\right)
$$

where for each $\gamma \in D$,

$$
I_{\gamma}=\left\{\begin{array}{cc}
{\left[0, b^{-k_{\gamma}}\right]} & \text { if } i_{k}=1 \\
\left(\left(i_{k}-1\right) b^{-k_{\gamma}}, i_{k} b^{-k_{\gamma}}\right] & \text { if } i_{k} \neq 1
\end{array}\right.
$$

The set $D$ is called the index set of $A$ and the sequence $\left\langle k_{\gamma}\right\rangle_{\gamma \in D}$ is called the exponent sequence of $A$. The set $[0, d]^{\alpha}$ is taken conventionally to be a multi$b^{k}$-tile with empty index set $\emptyset$. The index set of the multi- $b^{k}$-tile $A$ will be denoted by $D(A)$.

Lemma 8.4 implies: for $\gamma_{1}<\gamma_{2}<\ldots<\gamma_{n}<\alpha$, and Borel sets $B_{1}, B_{2}, \ldots, \dot{B}_{n}$ of $[0,1]$,

$$
\nu\left(\bigcap_{i=1}^{n} F_{\gamma_{i}}\left(B_{i}\right)\right)=\prod_{i=1}^{n} \lambda\left(B_{i}\right) .
$$

Easily, formula (8.9) implies the collection of $\sigma$-fields $\left\{\mathcal{F}_{=\gamma}: \gamma<\alpha\right\}$ is a totally independent collection of $\sigma$-algebras with respect to $\nu$. Further, (8.9) implies

$$
\nu(A)=b^{-\sum_{\gamma \in D(A)} k_{\gamma}}
$$

Lemma 8.5. The $\sigma$-algebra generated by all the multi- $b^{k}$-tiles and all the $\nu$-null sets is the $\sigma$-algebra of $\nu$-measurable sets. For any multi-b ${ }^{k}$-tile $A$ with index set $D(A)$ and exponent sequence $\left\langle k_{\gamma}\right\rangle_{\gamma \in D(A)}, \gamma<\alpha$, and any $\mathbf{s} \in A$,

$$
\log _{b}\left(\frac{\nu\left(A\left|\theta_{\alpha, \gamma}=\mathbf{s}\right|_{\gamma}\right)}{\nu(A)}\right)=\sum_{\beta<\gamma} k_{\beta}
$$

Proof. Easily $\mathcal{H}$ is measurable with respect to the $\sigma$-algebra generated by the multi$b^{k}$-tiles. The first statement follows from Lemma 8.3. Let $A=\bigcap_{\beta \in D} F_{\beta}\left(I_{\beta}\right)$ and $\gamma<\alpha$, and $D=D(A)$. Then $A=A^{\prime} \cap A^{\prime \prime}$ where $A^{\prime}=\bigcap_{\beta \in D: \beta<\gamma} F_{\beta}(\beta)$ and $A^{\prime \prime}=\bigcap_{\beta \in D: \beta \geq \gamma} F_{\beta}\left(I_{\beta}\right)$. Since the collection $\left\{\mathcal{F}_{=\beta}: \beta<\alpha\right\}$ is a totally independent collection of $\sigma$-fields with respect to $\nu$, Lemma 8.3 and (8.9) imply $\nu\left(A \mid \sigma\left(\theta_{\alpha, \gamma}\right)\right)=$ $\nu\left(A \mid H_{\beta} ; \beta<\gamma\right)=\prod_{\beta \in D: \beta \geq \gamma} \lambda\left(I_{\beta}\right) \cdot 1_{A^{\prime}}$. Thus, for $A$ a multi- $b^{k}$-tile with exponent sequence $\left\langle k_{\gamma}\right\rangle_{\gamma \in D(A)}$, for $\nu$-a.e.-s $\in A, \nu\left(A\left|\theta_{\alpha, \gamma}=\mathbf{s}\right|_{\gamma}\right)=\prod_{\beta \in D: \beta \geq \gamma} b^{-k_{\beta}}$. Since $\nu(A)=\prod_{\beta \in D} b^{-k_{\beta}},(8.11)$ follows.

We will now construct a positive filtration base.

Proposition 8.6. If $\kappa$ is a basic multispectrum of order $\alpha \geq 1$ with spreading measure $\nu$ and unidimensional map $U$, then there exists a positive filtration base $\left\langle\mathcal{P}_{n}\right\rangle_{n=0}^{\infty}$ which encodes a coordinate induction rule for $\kappa$. Further, the filtration base can be chosen so that for $\nu$-a.e.-u,

$$
\sum_{n=0}^{\infty}\left(\prod_{i=0}^{n} b^{-d^{-1} E_{\nu}\left[S_{0} \mid \mathcal{E}_{i}(\mathbf{u})\right]}\right) \frac{1}{\nu\left(\mathcal{E}_{n}(\mathbf{u})\right)}<\infty .
$$


Proof. For a fixed $\kappa$-setup, let $\delta_{1}, \delta_{2}, \ldots$ be a listing of the elements in $\alpha$, where the indexing is done with the natural numbers or a finite initial segment of the natural numbers. Further, choose the indexing so that $\delta_{1}=0$.

If $D$ is a finite subset of $\alpha$, then define the extension set, $D^{\prime}$, of $D$ by

$$
D^{\prime}=\left\{\begin{array}{cl}
D & \text { if } D=\alpha, \\
D \cup\left\{\delta_{i}\right\} & \text { if } D \neq \alpha \text { and } i=\inf \left\{j: \delta_{j} \notin D\right\} .
\end{array}\right.
$$

Let $\mathbb{Z}$ denote the integers. For each $n \geq 1$, define a function $g_{n}$ with domain $D_{0 m}=\left\{\left\langle A_{i}\right\rangle_{i=1}^{n}: \forall i \leq n, A_{i}\right.$ is a multi- $b^{k}$-tile and $\left.\forall i<n, A_{i} \supseteq A_{i+1}\right\}$ and with range the set $\{h: D \rightarrow \mathbb{Z}: D$ is a finite subset of $\alpha\}$ so that $g_{n}$ satisfies the following two conditions:

(1) The function $g_{n}\left(\left\langle A_{i}\right\rangle_{i=1}^{n}\right)$ has domain $D\left(A_{n}\right)^{\prime}$ where $D\left(A_{n}\right)$ is the index set for $A_{n}$ and ' is the extension operator.

(2) For $0=\gamma_{1}<\gamma_{2}<\cdots<\gamma_{m}$ the increasing listing of the elements of $D\left(A_{n}\right)^{\prime}$ and $\gamma_{m+1}=\alpha$, the elements of the integer valued function $g_{n}\left(\left\langle A_{i}\right\rangle_{i=1}^{n}\right)$ are defined by the following finite recursion formula: for $p \leq m$,

$$
\begin{aligned}
g_{n}\left(\left\langle A_{i}\right\rangle_{i=1}^{n}\right)\left(\gamma_{p}\right)=\max \{k & \in \mathbb{Z}: k+\sum_{j: j<p} g_{n}\left(\left\langle A_{i}\right\rangle_{i=1}^{n}\right)\left(\gamma_{j}\right) \\
& <d^{-1}\left(\sum_{i=1}^{n} E_{\nu}\left[S_{0} \mid A_{i}\right]-n \cdot E_{\nu}\left[S_{\gamma_{p+1}} \mid A_{n}\right]\right) \\
& \left.+\left(-3 \cdot 1_{E_{\nu}\left[S_{\gamma_{m}}-S_{\gamma_{m+1}} \mid A_{n}\right]>0}+1_{p=m}\right) \log _{b} n\right\} .
\end{aligned}
$$

For each $n \geq 1$, a function $G_{n}$ from $D o m_{n}$ into the collection of sets of multi- $b^{k}$ tiles is defined as follows: For $\left\langle A_{i}\right\rangle_{i=1}^{n} \in D o m_{n}$, if for all $\gamma \in D\left(A_{n}\right)^{\prime}, g_{n}\left(\left\langle A_{i}\right\rangle_{i=1}^{n}\right)(\gamma)$ $\geq 1+k_{\gamma} 1_{\gamma \in D\left(A_{n}\right)}$, then set $G_{n}\left(\left\langle A_{i}\right\rangle_{i=1}^{n}\right)=\left\{B\right.$ is a multi- $b^{k}$-tile : $B \subseteq A_{n}$, and $B$ has exponent sequence $\left.g_{n}\left(\left\langle A_{i}\right\rangle_{i=1}^{n}\right)\right\}$. Otherwise set $G_{n}\left(\left\langle A_{i}\right\rangle_{i=1}^{n}\right)=\left\{A_{n}\right\}$.

Let $\mathcal{P}_{0}=\left\{[0, d]^{\alpha}\right\}$. For each $n \geq 1$, set $\mathcal{P}_{n}=\left\{B: \exists\left\langle A_{i}\right\rangle_{i=1}^{n} \in \mathcal{P}_{0} \times \mathcal{P}_{1} \times \cdots \times \mathcal{P}_{n-1}\right.$ so that $\left.B \in G_{n}\left(\left\langle A_{i}\right\rangle_{i=1}^{n}\right)\right\}$. Let $Y=\left\{\left\langle A_{i}\right\rangle_{i=0}^{\infty}\right.$ : for each $i, A_{i} \in \mathcal{P}_{i}$ and $\left.A_{i} \supseteq A_{i+1}\right\}$. The set $Y$ is a compact Hausdorff space when given the topology of pointwise convergence. Since $G_{n}\left(\left\langle A_{i}\right\rangle_{i=1}^{n}\right)$ is always a collection of disjoint multi- $b^{k}$-tiles which unions to $A_{n}$, the following claim holds:

Claim 1. For each $n, \mathcal{P}_{n}$ is a finite partition of $[0, d]^{\alpha}$ into multi- $b^{k}$-tiles and $\mathcal{P}_{n+1}$ refined $\mathcal{P}_{n}$.

For any $\left\langle A_{i}\right\rangle_{i=0}^{\infty} \in Y$, the definition of $g_{n}$ implies

$$
\begin{aligned}
& d^{-1}\left(\sum_{i=1}^{n} E_{\nu}\left[S_{0} \mid A_{i-1}\right]-n \cdot E_{\nu}\left[S_{\gamma_{p+1}} \mid A_{n-1}\right]\right) \\
& +\left(-3 \cdot 1_{E_{\nu}\left[S_{\gamma_{m}}-S_{\gamma_{m+1}} \mid A_{n-1}\right]>0}+1_{p=m}\right) \log _{b} n \\
& +\left(-\sum_{j \leq p} g_{n}\left(\left\langle A_{k-1}\right\rangle_{k=1}^{n}\right)\left(\gamma_{j}\right)\right) \in[0,1) .
\end{aligned}
$$

In particular for $p=1$,

$$
\begin{aligned}
g_{n}\left(\left\langle A_{k-1}\right\rangle_{k=1}^{n}\right)\left(\gamma_{1}\right) & \geq-1+d^{-1}\left(\sum_{i=1}^{n} E_{\nu}\left[S_{0} \mid A_{i-1}\right]-n \cdot E_{\nu}\left[S_{\gamma_{2}} \mid A_{n-1}\right]\right) \\
& +\left(-3 \cdot 1_{E_{\nu}\left[S_{\gamma_{m}}-S_{\gamma_{m+1}} \mid A_{n-1}\right]>0}+1_{1=m}\right) \log _{b} n
\end{aligned}
$$


and for $p \geq 2$, subtracting the left hand side of (8.13) with $p-1$ substituted for $p$ from the left hand side of (8.13) (without changing $p$ ) and noting the result must lie in $(-1,1)$, it follows that

$$
g_{n}\left(\left\langle A_{k-1}\right\rangle_{k=1}^{n}\right)\left(\gamma_{p}\right) \geq-1+n d^{-1} E_{\nu}\left[S_{\gamma_{p}}-S_{\gamma_{p+1}} \mid A_{n}\right]+\left(\log _{b} n\right) \cdot 1_{p=m} .
$$

Claim 2. For each $\left\langle A_{i}\right\rangle_{i=0}^{\infty} \in Y$, for infinitely many $i, A_{i} \neq A_{i+1}$.

Proof of Claim 2 via contradiction: Suppose that $\left\langle A_{i}\right\rangle_{i=0}^{\infty}$ is eventually constant, that is, there is a integer $N$, so that for all $n \geq N, A_{n}=A_{N}$. There are two cases:

Case 1: $S_{0}=S_{\gamma_{2}} \nu$-a.s.

In this case, the order of $\kappa$ must be 1 and $\gamma_{1}=0$ and $\gamma_{2}=1$. (Since $\kappa$ is a basic multispectrum of order $\alpha \geq 1$, there is a strict descending of the $S_{i}$ until the unidimensional map is reached. See Lemma 8.1.) Thus $\alpha=1=p=m$, and the right hand side of the inequality (8.14) increases like $\log _{b} n$. Since $\alpha=1=\{0\}=$ $D\left(A_{N}\right)^{\prime}, g_{n}\left(\left\langle A_{i}\right\rangle_{i=1}^{n}\right)(\gamma) \geq 1+k_{\gamma} 1_{\gamma \in D\left(A_{n}\right)}$ eventually and so the set $A_{N}$ must split for large enough $n$. Contradiction.

Case 2: The $\nu$-probability that $S_{0} \neq S_{\gamma_{2}}$ is positive.

This hypothesis together with Lemma 8.1 implies $E_{\nu}\left[S_{0}-S_{\gamma_{2}} \mid A_{n}\right]>0$. Thus, the right hand side of (8.14) is increasing of a linear order. In fact, Lemma 8.1 implies if $0<p<m$, then $\nu$-a.s., $S_{\gamma_{p}}-S_{\gamma_{p+1}}>0$. Thus the right hand side of (8.15) is increasing of a linear order, for $0<p<m$. If $p=m$, then $S_{\gamma_{p}}-S_{\gamma_{p+1}} \geq 0$ and the right hand side of (8.15) tends to infinity at least of order $\log _{b} n$ if $m>\gamma_{2}$ and of a linear order if $m=\gamma_{2}$. This implies that for some large $n$, for all $\gamma$, $g_{n}\left(\left\langle A_{i}\right\rangle_{i=1}^{n}\right)(\gamma) \geq 1+k_{\gamma} 1_{\gamma \in D\left(A_{n}\right)}$ implying that a split has to occur. Contradiction. Claim 2 is established.

Claim 3. For each $\left\langle A_{i}\right\rangle_{i=0}^{\infty} \in Y$ and for each $\gamma<\alpha$, there is a $N(\gamma)$ so that for all $n \geq N(\gamma), \gamma \in D\left(A_{n}\right)$.

Proof of Claim 3: There is a integer $j$ so that $\gamma=\delta_{j}$. By the definition of $\mathcal{P}_{n}$, $\delta_{j} \in D\left(A_{n}\right)$, if there is at least $j i$ 's less than $n$ so that $A_{i} \neq A_{i+1}$. Apply Claim 2 .

Claim 4. $\sigma-\left(\bigcup_{n=0}^{\infty} \mathcal{P}_{n}\right)$ is almost the $\nu$-measurable sets.

Proof of Claim 4: Let $A$ be a multi- $b^{k}$-tile with index set $D(A)$ and exponent sequence $\left\langle k_{\gamma}\right\rangle_{\gamma \in D(A)}$. By Claim 3, for each $\left\langle A_{i}\right\rangle_{i=0}^{\infty} \in Y$, there is a $N$ so that for each $n \geq N, D(A) \subseteq D\left(A_{n}\right)$. Since each time $A_{n} \neq A_{n+1}$, the exponents $k_{\gamma}$ with index $\gamma \in D\left(A_{n}\right)$ increase by at least 1 , there is a $M$ depending on $\left\langle A_{i}\right\rangle_{i=0}^{\infty}$ so that for all $n \geq M$, both $D(A) \subseteq D\left(A_{n}\right)$ and for all $\gamma \in D(A), k_{\gamma}\left(A_{n}\right) \geq k_{\gamma}$ where $\left\langle k_{\gamma}\left(A_{n}\right)\right\rangle_{\gamma \in D\left(A_{n}\right)}$ is the exponent sequence of $A_{n}$. This implies for $n \geq M$, either $A_{n} \subseteq A$ or $A_{n} \cap A=\emptyset$. Using the compactness of $Y$, it is easy to see that there is an integer $n$ so that for all $B \in \mathcal{P}_{n}$ either $B \subseteq A$ or $B \cap A=\emptyset$. Easily $A \in \sigma\left(\mathcal{P}_{n}\right)$ for that $n$. Thus $\sigma\left(\bigcup_{n=0}^{\infty} \mathcal{P}_{n}\right)$ contains all the multi- $b^{k}$-tiles. The claim follows from the first statement of Lemma 8.5.

Since every multi- $b^{k}$-tile has positive $\nu$ measure, Claims 1 and 4 together imply the following claim:

Claim 5. $\left\langle\mathcal{P}_{n}\right\rangle_{n=0}^{\infty}$ is a positive filtration base for $\kappa$.

Claim 6. The positive filtration base $\left\langle\mathcal{P}_{n}\right\rangle_{n=0}^{\infty}$ encodes a coordinate induction rule for $\kappa$. 
Proof of Claim 6: Since $\sigma\left(\bigcup_{n=0}^{\infty} \mathcal{P}_{n}\right)$ is almost the $\nu$-measurable sets, for all $\gamma<\alpha, E_{\nu}\left[S_{\gamma} \mid \sigma\left(\mathcal{P}_{n}\right)\right]$ converges to $S_{\gamma}, \nu$-a.s. and $E_{\nu}\left[S_{\alpha} \mid \sigma\left(\mathcal{P}_{n}\right)\right]$ converges to $U$, $\nu$-a.s. Let $I N D$ be the set of $\mathbf{s} \in[0, d]^{\alpha}$ at which these two limits exists.

Fix $\gamma<\alpha$ and $\mathbf{s} \in I N D$. Since $\left\langle\mathcal{E}_{n}(\mathbf{s})\right\rangle_{n=0}^{\infty} \in Y$, by Claim 3, there is a $N(\gamma)$ so that for all $n \geq N(\gamma), \gamma \in D\left(\mathcal{E}_{n}(\mathbf{s})\right)$. Let $I(\mathbf{s})=\left\{n \geq N(\gamma): \mathcal{E}_{n}(\mathbf{s}) \neq \mathcal{E}_{n-1}(\mathbf{s})\right\}$. From claim 3 we know $I(\mathbf{s})$ is infinite. If $n+1 \notin I(\mathbf{s})$, then $\mathcal{E}_{n+1}(\mathbf{s})=\mathcal{E}_{n}(\mathbf{s})$ implying

$$
\frac{1}{n} \log _{b}\left(\frac{\nu\left(\mathcal{E}_{n}(\mathbf{s})\left|\theta_{\alpha, \gamma}=\mathbf{s}\right|_{\gamma}\right)}{\nu\left(\mathcal{E}_{n}(\mathbf{s})\right)}\right) \geq \frac{1}{n+1} \log _{b}\left(\frac{\nu\left(\mathcal{E}_{n+1}(\mathbf{s})\left|\theta_{\alpha, \gamma}=\mathbf{s}\right|_{\gamma}\right)}{\nu\left(\mathcal{E}_{n+1}(\mathbf{s})\right)}\right)
$$

Thus,

$$
\begin{aligned}
& \limsup _{n \rightarrow \infty} \frac{1}{n} \log _{b}\left(\frac{\nu\left(\mathcal{E}_{n}(\mathbf{s})\left|\theta_{\alpha, \gamma}=\mathbf{s}\right|_{\gamma}\right)}{\nu\left(\mathcal{E}_{n}(\mathbf{s})\right)}\right) \\
& \quad=\limsup _{\substack{n \rightarrow \infty \\
n \in I(\mathbf{s})}} \frac{1}{n} \log _{b}\left(\frac{\nu\left(\mathcal{E}_{n}(\mathbf{s})\left|\theta_{\alpha, \gamma}=\mathbf{s}\right|_{\gamma}\right)}{\nu\left(\mathcal{E}_{n}(\mathbf{s})\right)}\right) .
\end{aligned}
$$

But when $n \in I(\mathbf{s})$, the formula (8.11) and the definition of $G_{n}$ imply

$$
\begin{aligned}
\frac{1}{n} \log _{b}\left(\frac{\nu\left(\mathcal{E}_{n}(\mathbf{s})\left|\theta_{\alpha, \gamma}=\mathbf{s}\right|_{\gamma}\right)}{\nu\left(\mathcal{E}_{n}(\mathbf{s})\right)}\right) & =n^{-1} \cdot \sum_{\beta \in V_{n}} k_{\beta}\left(\mathcal{E}_{n}(\mathbf{s})\right) \\
& =n^{-1} \cdot \sum_{\beta \in V_{n}} g_{n}\left(\left\langle\mathcal{E}_{i-1}(\mathbf{s})\right\rangle_{i=1}^{n}\right)(\beta)
\end{aligned}
$$

where $V_{n}=\left\{\beta \in D\left(\mathcal{E}_{n}(\mathbf{s})\right): \beta<\gamma\right\}$. By (8.13) and the fact $\mathbf{s} \in I N D$,

$$
\lim _{\substack{n \rightarrow \infty \\ n \in I(\mathbf{s})}} n^{-1} \cdot \sum_{\beta \in V_{n}} g_{n}\left(\left\langle\mathcal{E}_{i-1}(\mathbf{s})\right\rangle_{i=1}^{n}\right)(\beta)=d^{-1}\left(s_{0}-s_{\beta}\right)
$$

(8.16), (8.17), and (8.18) together imply the first statement of (7.2). A similar argument shows that the second statement of (7.2) is true. Thus the claim is proved.

All that is left is to prove (8.12). As noted before $\nu$-a.s., $\lim _{n \rightarrow \infty} E_{\nu}\left[S_{0} \mid \sigma\left(\mathcal{P}_{n}\right)\right]=$ $S_{0}$. Since $\left\langle\mathcal{P}_{n}\right\rangle_{n=0}^{\infty}$ encodes a coordinate induction rule, the second equation of (7.2) and the preceding statement imply for $\nu$-a.e.-u,

$$
\lim _{n \rightarrow \infty} \frac{1}{n} \log _{b} \prod_{i=0}^{n} b^{-d^{-1} E_{\nu}\left[S_{0} \mid \mathcal{E}_{i}(\mathbf{u})\right]} \cdot \frac{1}{\nu\left(\mathcal{E}_{n}(\mathbf{u})\right)}=-d^{-1} U(\mathbf{u}) .
$$

Thus the statement (8.12) follows for $\nu$-a.e.-u $\in\{\mathbf{s}: U(\mathbf{s})>0\}$.

Now consider the case where $\mathbf{s} \in I N D$ and $U(\mathbf{s})=0$. Note that $S_{0}>0, \nu$-a.s., since the diffuseness order of $\kappa$ is $\geq 1$. We suppose $s_{0}=S_{0}(\mathbf{s})>0$. Fix $0<z<s_{0}$. There exists a $N(\mathbf{s})$ so that for all $n \geq N(\mathbf{s}), E_{\nu}\left[S_{0} \mid \mathcal{E}_{n}(\mathbf{s})\right]>z$. Further for such $n$, if $\mathcal{E}_{n}(\mathbf{s}) \neq \mathcal{E}_{n-1}(\mathbf{s})$, then statement (8.13) for $p=m+1$ implies

$$
\left(\prod_{i=0}^{n} b^{-d^{-1} E_{\nu}\left[S_{0} \mid \mathcal{E}_{i}(\mathbf{s})\right]}\right) \frac{1}{\nu\left(\mathcal{E}_{n}(\mathbf{s})\right)} \leq n^{-2} .
$$

If $\mathcal{E}_{n}(\mathbf{s})=\mathcal{E}_{n-1}(\mathbf{s})=\ldots=\mathcal{E}_{n-i}(\mathbf{s}) \neq \mathcal{E}_{n-i-1}(\mathbf{s})$, then

$$
\left(\prod_{i=0}^{n} b^{-d^{-1} E_{\nu}\left[S_{0} \mid \mathcal{E}_{i}(\mathbf{s})\right]}\right) \frac{1}{\nu\left(\mathcal{E}_{n}(\mathbf{s})\right)} \leq b^{1-i z}(n-i)^{-2} \text {. }
$$


A comparison test with $\sum C(\mathbf{s}) n^{-2}$ for some $C(\mathbf{s})$ shows convergence.

Proposition 8.7. If $\kappa$ is a basic multispectrum of order $\alpha \geq 1$ and with spreading measure $\nu$, so that $\kappa$-a.s. $S_{0} \leq d$, then there exists a coordinate induction pair $\left\langle\left\langle\mathcal{P}_{n}\right\rangle_{n=0}^{\infty}, r\right\rangle$ for $\kappa$ for which

$$
\sum_{n=0}^{\infty} b^{-n}\left(\prod_{i=0}^{n} r\left(\mathcal{E}_{i}(\mathbf{s})\right)\right) \frac{1}{\nu\left(\mathcal{E}_{i}(\mathbf{s})\right)}<\infty .
$$

Proof. Let $\left\langle\mathcal{P}_{n}\right\rangle_{n=0}^{\infty}$ be a positive filtration base guaranteed by Proposition 8.6. Since the order of $\kappa$ is greater than $0, \kappa$-a.s., $S_{0}$ is non-atomically distributed. Thus, $d>E_{\nu}\left[S_{0} \mid \mathcal{E}_{i}(\mathbf{s})\right]>0$ for any $\mathbf{s}$ and $i$. Thus there exists uncountably many 1-1 functions, $r$, with range in $\{0\} \cup[1, b]$, such that $r(\emptyset)=0$, and for all $\mathbf{s} \in[0, \infty]^{\alpha}$, and $i$,

$$
\log _{b}\left(r\left(\mathcal{E}_{i}(\mathbf{s})\right)\right) \in\left[\frac{i}{i+1}\left(1-d^{-1} E_{\nu}\left[S_{0} \mid \mathcal{E}_{i}(\mathbf{s})\right]\right), 1-d^{-1} E_{\nu}\left[S_{0} \mid \mathcal{E}_{i}(\mathbf{s})\right]\right) .
$$

Any such $r$ is a transfer map for $\left\langle\mathcal{P}_{n}\right\rangle_{n=0}^{\infty}$ and (8.19) follows from (8.12). But for $\nu$-a.e.-s, $E_{\nu}\left[S_{0} \mid \mathcal{E}_{i}(\mathbf{s})\right]$ converges to $s_{0}$. Thus $\log _{b}\left(r\left(\mathcal{E}_{i}(\mathbf{s})\right)\right)$ converges to $1-\frac{s_{0}}{d}$ $\nu$-a.s.

\section{The Multispectral Theorem in $\mathbf{R}^{n}$ : Necessary and Sufficient Conditions}

Theorem 9.1. Let $T=\{0, \ldots, b-1\}^{\mathbf{N}}$ and $\rho_{d}$ be as before and $\kappa$ be a finite regular Borel measure on $[0, \infty]^{\omega_{1}}$. If $\kappa$ satisfies both

(1) $\kappa$-a.s.-s, $s_{0} \leq d$, and

(2) there exist a sequence of non-negative real numbers, $\left\langle a_{i}\right\rangle_{i=1}^{\infty}$, and a sequence of basic multispectrums, $\left\langle\kappa_{i}\right\rangle_{i=1}^{\infty}$, so that $\sum_{i=1}^{\infty} a_{i}<\infty$ and $\kappa=\sum_{i=1}^{\infty} a_{i} \kappa_{i}$.

Then $\kappa \in \mathcal{S}_{\left(T, \rho_{d}\right)}$.

Proof. Fix a $\kappa_{i}$ of condition (2). If $\kappa_{i}$ is of spreading order $\geq 1$, then by Theorem 7.7 and Proposition 8.7, there is a measure valued process $\left\langle\lambda_{n}\right\rangle_{n=0}^{\infty}$ so that

$$
P \text {-a.s., } \sigma_{\omega_{1}}\left(\lambda_{\infty}\right) \ll \kappa
$$

and if $B$ is a Borel subset of $[0, \infty]^{\omega_{1}}$ with $\kappa(B)>0$, then $P\left(\sigma_{\omega_{1}}\left(\lambda_{\infty}\right)(B)>0\right)>0$. It follows from Theorem 6.6 that $\kappa_{i} \in \mathcal{S}_{\left(T, \rho_{d}\right)}$. If $\kappa_{i}=\delta_{x}$ for $x \in(0, d]$, then using the main theorem of say [11], there is a random unidimensional measure of dimension $\frac{x}{d}$ with respect to the ultrametric $\rho_{1}$. Using (7.1), this last implies there is a random unidimensional measure of dimension $x$ with respect to $\rho_{d}$. The case $\kappa_{i}=\delta_{0}$ is trivial since $T$ is non-empty. Thus for all $i, \kappa_{i} \in \mathcal{S}_{\left(T, \rho_{d}\right)}$. Since $\mathcal{S}_{\left(T, \rho_{d}\right)}$ is a strongly closed cone, $\kappa_{i} \in \mathcal{S}_{(T, \rho)}$ for all $i$ implies $\sum_{i=1}^{\infty} a_{i} \kappa_{i}$ is an element of $\mathcal{S}_{\left(T, \rho_{d}\right)}$.

Theorem 9.2 (Multispectral Theorem). Let d be a positive integer. The following three conditions $(1,2,3)$ are equivalent:

(1) $\kappa$ is a multispectrum of a Borel measure on $[0,1]^{d}$ (i.e., $\kappa \in S_{[0,1]^{d}}$ ).

(2) The statements (a) and (b) hold:

(a) $\kappa=\sum_{i=1}^{\infty} c_{i} \kappa_{i}$ where $\left\langle c_{i}\right\rangle_{i=1}^{\infty}$ is a sequence of non-negative real numbers with $\sum_{i=1}^{\infty} c_{i}<\infty$ and $\left\langle\kappa_{i}\right\rangle_{i=1}^{\infty}$ is a sequence of basic multispectrums, and (b) $\kappa$-a.e.-s, $s_{0} \leq d$.

(3) The statements (c), (d), and (e) hold: 
(c) for all $\beta<\alpha<\omega_{1}$, $\kappa$-a.e.-s, $s_{\beta} \geq s_{\alpha}$,

(d) for each countable ordinal $\beta$, for $\kappa \circ \rho_{\beta}^{-1}$-a.e.-s, for every $x \in[0, \infty]$, if $x$ is an atom of $\left(\kappa \circ \rho_{\beta+1}^{-1}\right)_{\mathbf{s}}$, then $\kappa_{\rho_{\beta+1}=\mathbf{s} * x}=\delta_{\mathbf{s} * \widehat{x}}$ where $\mathrm{s} \rightarrow\left(\kappa \circ \rho_{\beta+1}^{-1}\right)_{\mathbf{s}}$,' is the factor kernel of $\kappa \circ \rho_{\beta+1}^{-1}$ with respect to $\theta_{\beta+1, \beta}$, and $\mathbf{u} \rightarrow \kappa_{\rho_{\beta+1}=\mathbf{u}}$ ', is the decomposition kernel of $\kappa$ with respect to $\rho_{\beta+1}$.

(e) $\kappa$-a.e.-s, $s_{0} \leq d$.

Proof. First we show (1) implies (3). Since $[0,1]^{d}$ has dimension $d$, (e) holds. But (c) and (d) follow from Theorem 4.3. Easily (3) implies (2) since (e) implies (b) and Lemma 5.1 along with (c) and (d) implies (a). Lastly, we show (2) implies (1).

Let $T=\left\{0,1, \ldots, b^{d}-1\right\}^{\mathbb{N}}$ with ultrametic $\rho_{d}$. By Theorem 9.1, there exists a finite Borel measure $\mu \in M^{+}(T)$ such that $\sigma_{\omega_{1}}(\mu)=\kappa$. Let $F: T \rightarrow[0,1]^{d}$ be the function defined by

$$
F\left(\left\langle x_{i}\right\rangle_{i=1}^{\infty}\right)=\left\langle\sum_{n=1}^{\infty} b^{-n} x_{n, j}\right\rangle_{j=1}^{d}
$$

where $x_{n, d} x_{n, d-1} \cdots x_{n, 1}$ is the base $b$ expansion of $x_{n}$ (with zeros added to the left if needed to expand out to $\left.x_{n, d}\right)$. Now, $\mu \circ F^{-1} \in M^{+}\left([0,1]^{d}\right)$. It follows from the net comparison theorems of Rogers [16] that for any Borel $A$ in $T$ and Borel $B$ in $[0,1]^{d}$, the dimension of $F(A)$ in $[0,1]^{d}$ is the same as the $\rho_{d^{-}}$-dimension of $A$, and the $\rho_{d^{-}}$dimension of $F^{-1}(B)$ is the same as the dimension of $B$ in $[0,1]^{d}$. Thus $\sigma_{\omega_{1}}\left(\mu \circ F^{-1}\right)=\sigma_{\omega_{1}}(\mu)=\kappa$.

Remark 9.1. At this point we can prove the converse of Lemma 5.1. Let $T$ denote the Hilbert cube $[0,1] \times\left[0, \frac{1}{2}\right] \times\left[0, \frac{1}{3}\right] \times \cdots \times\left[0, \frac{1}{n}\right] \times \cdots$ with the metric

$$
\rho\left(\left\langle x_{n}\right\rangle_{n=1}^{\infty},\left\langle y_{n}\right\rangle_{n=1}^{\infty}\right)=\sqrt{\sum_{n=1}^{\infty}\left(x_{n}-y_{n}\right)^{2}} .
$$

Since for each $d \in \mathbb{N}$, the subspace $T_{d}=\left\{\left\langle x_{n}\right\rangle_{n=1}^{\infty} \in T\right.$ : for $n=1, \ldots, d, x_{n} \in\left[0, \frac{1}{d}\right]$, for $\left.n \geq d+1, x_{n}=0\right\}$ of $T$ is an isometric copy of $\left[0, \frac{1}{d}\right]^{d}$ with respect to the projection map of $T$ onto its first $d$ coordinates. Thus if $\kappa$ satisfies the conditions of (2) for any $d$, then $\kappa$ is in $\mathcal{S}_{(T, \rho)}$. But if $\mu$ is the infinite product measure of $\prod \mu_{i}$ where each factor $\mu_{i}$ is the normalized Lebesgue measure on $[0, i]$, then $\sigma_{\omega_{1}}(\mu)=\delta_{\infty}$. Thus every measure of the form of the conclusion of Lemma 5.1 is in $\mathcal{S}_{(T, \rho)}$. Thus every measure of the form of the conclusion of Lemma 5.1 has the form of the hypothesis of Lemma 5.1 by Theorem $4.3 .^{8}$

Remark 9.2. It is also true, but will not be shown here, that Theorem 9.2 is true for Borel sets in the following modified sense. For every Borel subset $B$ of $[0,1]^{d}$, then Theorem 9.2 is true if both the lines (b) and (e) of the theorem are replaced by one the following lines:

(1) $\kappa$-a.s., $S_{0} \leq \operatorname{dim}(B)$,

(2) $\kappa$-a.s., $S_{0}<\operatorname{dim}(B)$,

where (1) is chosen if there is a finite Borel measure on $B$ which is unidimensional of dimension $\operatorname{dim}(B)$, otherwise (2) is chosen.

\footnotetext{
${ }^{8}$ It is also fairly easy to show that the converse of Lemma 5.1 is true directly without going through Theorem 9.2 and Theorem 4.3.
} 
Remark 9.3 (Concluding). We conclude with the following remarks:

Let $T$ be a compact metric space, and $M(T)^{*}$ denote the linear functionals on the bounded countably additive Borel signed measures of $T$ which are strongly continuous. Let $|\mu|$ denote the total variation measure of $\mu$ for $\mu \in M^{+}(T)$. The following Representation Theorem can be found in Conway [3] (Theorem 5.12) and is of the type that Mauldin attributes to Schreier in [12].

Theorem. If $\psi^{*} \in M(T)^{*}$, then there exists a bounded function $\Phi_{\psi^{*}}: M(T) \times T \rightarrow$ $\mathbb{R}$ so that for all finite signed measures $\mu$ and $\nu, 1) \Phi_{\psi^{*}}(\mu, \cdot)$ is $|\mu|$-measurable, 2$)$ if $|\nu| \ll|\mu|$, then $|\nu|$-a.e., $\Phi_{\psi^{*}}(\nu, \cdot)=\Phi_{\psi^{*}}(\mu, \cdot)$ and 3) $\psi^{*}(\mu)=\int \Phi_{\psi^{*}}(\mu, t) d \mu(t)$.

Such $\Phi_{\psi^{*}}$ can be thought of as a local parameter map for the functional $\psi^{*}$. It follows from Lemma 6.2 that the first order parameter extension map $K_{\psi^{*}}$ : $M(T) \rightarrow M(T \times \mathbb{R})$ defined by

$$
K_{\psi^{*}}(\mu)=\mu \circ\left\langle i d, \Phi_{\psi^{*}}(\mu, \cdot)\right\rangle^{-1}
$$

is linear and strongly continuous. However, $K_{\psi^{*}}$ may not have enough weak* measurability to form the transfinite parameter extensions of Definition 3.2 since the iterated integrals needed will not be well-defined. ${ }^{9}$

A function $\psi$ from the Borel sets of $T$ into the non-negative real numbers is called an increasing set function if it satisfies condition (1) of Section 2. (Examples: $\psi(A)=$ Hausdorff dimension of $A, \psi(A)=$ the packing dimension of $A, \psi(A)=$ the irregularity coefficient of $A$ in the cases where the Hausdorff dimension and packing dimension of $T$ are finite. If $F: T \rightarrow[0, \infty)$ is bounded Borel, then $\psi(A)=\sup \{F(x): x \in A\}$ also defines such a set function.) For such increasing set functions, a positive linear functional $\psi^{*}$ on $M^{+}(T)$ can be defined by the formula

$$
\psi^{*}(\mu)=\inf _{\mathcal{P}} \sum_{A \in \mathcal{P}} \psi(A) \mu(A)
$$

where the inf is over all finite Borel partitions of $T$. Let $\|\cdot\|_{\mu, \infty}$ denote the essential supremum norm with respect to $\mu$. If $\hat{\psi}$ is defined by

$$
\hat{\psi}(A)=\sup _{\mu \in M^{+}(T)}\left\|1_{A} \Phi_{\psi^{*}}(\mu, \cdot)\right\|_{\mu, \infty} 10
$$

then it is not hard to show that when $\psi$ is an increasing set function, $\hat{\psi}(A)$ satisfies (1) and (2) of page $2^{11}, \hat{\psi} \leq \psi$, and $\hat{\psi}^{*}=\psi^{*}$. In this case the local parameter function $\Phi_{\psi^{*}}$ satisfies all the statements of the first order theory (Theorems 2.1, 2.2 , and 2.3) except possibly the statement that $\Phi_{\psi^{*}}$ is weakly Borel. If $\Phi_{\psi^{*}}$ and $K_{\psi^{*}}$ are sufficiently measurable, then the iterated integrals of the definitions of the transfinite multiparameter extensions are well defined and the theorems of Sections $3,4,5$, and 6 are true. However the converse of Theorem 4.3 need not be true for these increasing parameters. (Consider the example $F$ given above.)

\footnotetext{
${ }^{9}$ For example: Let $T=[0,1]$, let $\lambda$ denote Lebesgue measure on $[0,1]$, and let $A$ be a subset of $[0,1]$ not measurable with respect to $\lambda$. Define $\psi^{*}(\mu)=\int x g_{\mu}(x) d \lambda+\sum_{x \in A} x \mu(\{x\})$, where $g_{\mu}(x) d \lambda$ is the part of the Lebesgue decomposition of $\mu$ which is absolutely continuous with respect to $\lambda$. In this case $K_{\psi^{*}}(\lambda)=w^{*}-\int \delta_{x} \times \delta_{x} d \lambda(x)$ and $K_{\psi^{*}}\left(\delta_{x}\right)=1_{A}(x) \delta_{x} \times \delta_{x}+1_{A^{c}}(x) \delta_{x} \times \delta_{0}$ but $\iint 1_{y>0} d K_{\psi^{*}}\left(\delta_{x}\right)(t, y) d \lambda(x)$ is not well defined.

${ }^{10}$ Potential/capacitance theory with this type of formula can be found elsewhere, for example in [5]. Our interest here is merely the the line $\hat{\psi}^{*}=\psi^{*}$.

${ }^{11}$ Tricot gives some discussion on " $\sigma$-stability" and also of the "irregularity of a set" in [17].
} 
On the other hand, let $T=[0,1]^{d}$ with the Euclidean metric. For each ordinal $\alpha, 0<\alpha<\omega_{1}$, the collection of maps $\left\langle\phi_{\mu, \alpha}\right\rangle_{\mu \in M^{+}(T)}$ of Theorem 3.1 (3.4) defines a positive linear functional, $\psi_{\alpha}^{*}$, the $\alpha$-level dimension moment functional, on $M^{+}(T)$ by the formula

$$
\psi_{\alpha}^{*}(\mu)=\int \inf \phi_{\mu, \alpha}(t) d \mu(t)
$$

where inf $\mathbf{s}=\inf _{\beta<\alpha} s_{\beta}$ if $\mathbf{s} \in[0, \infty]^{\alpha}$. There is also a unidimensionality moment functional, $\psi_{\omega_{1}}^{*}$, defined by

$$
\psi_{\omega_{1}}^{*}=\lim _{\alpha \rightarrow \omega_{1}} \psi_{\alpha}^{*}
$$

The theorems of Sections 3, 4, 5, and 6 are true for the resulting transfinite sequence. And if $\alpha<\omega_{1}$, even Theorem 9.2 is true. However, for no $\alpha>1$, can $\psi_{\alpha}^{*}$ be expressed in terms $\Psi^{*}$ for some increasing set parameter $\Psi$. In fact, if $\delta \in[0, \psi(A)$ ), then there exists a Borel set, $B$, of finite positive $\delta$-Hausdorff measure so that $B \subseteq A$. See Rogers [16]. Let $\mu_{\delta}$ be defined by

$$
\mu_{\delta}(C)=\mathcal{H}^{\delta}(C \cap B)
$$

where $\mathcal{H}^{\delta}$ then it is not hard to show $\mu_{\delta}$ is unidimensional of dimension $\delta$, and so $\Phi_{\psi_{\alpha}^{*}}(\mu, \cdot)=\delta$ a.s., implying $\sup _{\mu \in M^{+}(T)}\left\|1_{A} \Phi_{\psi_{\alpha}^{*}}(\mu, \cdot)\right\|_{\mu, \infty} \geq \psi(A)$, the capacitarian dimension of $A$, for all $\alpha$. But $1_{A} \Phi_{\psi_{\alpha}^{*}}(\mu, \cdot) \leq \psi(A) \mu$-a.s. for all $\mu \in M^{+}(T)$. Thus,

$$
\sup _{\mu \in M^{+}(T)}\left\|1_{A} \Phi_{\psi_{\alpha}^{*}}(\mu, \cdot)\right\|_{\mu, \infty}=\psi(A) .
$$

So only $\psi_{1}^{*}$ is generated by an increasing set function. That the more general formula $\psi_{\alpha}^{*}(\mu)=\lim _{\mathcal{P}} \sum_{A \in \mathcal{P}} \psi_{\alpha}(A) \mu(A)$ holds for some bounded (although not an increasing) set function $\psi_{\alpha}, \alpha>1$, follows from a theorem of Mauldin under certain set-theoretic assumptions.

\section{REFERENCES}

1. Paul Bernays. Axiomatic Set Theory. Dover Publications, New York, 1991. MR 92a:04001.

2. Donald L. Cohn. Measure Theory. Birkhäuser, Boston, 1980. MR 81k:28001.

3. John B. Conway. A Course in Functional Analysis. Springer-Verlag, New York, second edition, 1990. p. 76. MR 91e:46001.

4. Collen Cutler. The Hausdorff dimension distribution of finite measures in euclidean space. Canandian Journal of Mathematics, XXXVIII(6):1459-1484, 1988. MR 88b:28013.

5. Joseph L. Doob. Classical Potential Theory and Its Probabilistic Counterpart. SpringerVerlag, New York, 1984. MR 85k:31001.

6. R. Holley and E.C. Waymire. Multifractal dimension and scaling exponents for strongly bounded random cascades. The Annals of Applied Probability, 2:819-845, 1992. MR 93k:60122

7. J.P. Kahane. Some Random Series of Functions. Cambridge University Press, New York, second edition, 1985. MR 87m:60119.

8. J.P. Kahane. Multiplications aléatoires et dimension de Hausdorff. Ann. Inst. Poincaré, 23:112, 1987. MR 88h:60100.

9. J.P. Kahane. Positive martingales and random measures. Chinese Ann. Math., 8b:1-12, 1987. MR 88j:60098.

10. J.P. Kahane and Y. Katznelson. Décomposition des mesures selon la dimension. Colloq. Math., LVIII:269-279, 1990. MR 91g:28018.

11. J.P. Kahane and Jaques Peyrière. Sur certaines martingales de Benoit Mandelbrot. Advances in Mathematics, 22:131-145, 1976. MR 55:4355. 
12. R. Daniel Mauldin. A representation theorem for the second dual of C[0,1]. Studia Mathematica, XLVI:197-200, 1973. MR 49:11231.

13. G. Brown, G. Michon and Jaques Peyrière. On the multifractal analysis of measures. J. Statist. Phys. 66:775-790 (1992), MR 93c:58120.

14. L. Olsen. Multifractal formalism. Adv. in Math. 116:82-196 (1995), CMP 1996:4.

15. K.R. Parthasarthy. Introduction to Probability and Measure. Springer-Verlag, New York, 1978. MR 58:31322b.

16. C.A. Rogers. Hausdorff Measures. Cambridge University Press, 1970. MR 43:7576.

17. Claude Tricot, Jr. Two definitions of fractional dimension. Mathematical Proceedings of the Cambridge Philosophical Society, 91:57-74, 1982. MR 84d:28013.

18. E.C. Waymire and S.C. Williams. Multiplicative cascades: Dimension spectra and dependence. Journal of Fourier Analysis and its Applications. 1995, Special issue, 589-609. CMP 1996:5.

19. E.C. Waymire and S.C.Williams. Markov cascades. IMA Volume on Braveling Processes, ed. by K. Athroya and P. Jagers (in press).

20. E.C. Waymire and S.C. Williams. A general cascade decomposition theory. Bulletin (New Series) of the American Mathematical Society, 31:216-222 (1994). MR 95a:60065.

21. E.C. Waymire and S.C. Williams. A cascade decomposition theory with applications to Markov and exchangeable cascades. Transactions of the American Mathematical Society. 348:585-632 (1996). CMP 1995:10

Department of Mathematics and Statistics, Utah State University, Logan, Utah 84322-3900

E-mail address: williams@sunfs.math.usu.edu 